\title{
Sall1 is a Transcriptional Regulator Defining Microglia Identity and Function
}

\author{
Dissertation \\ zur \\ Erlangung der naturwissenschaftlichen Doktorwürde \\ (Dr. sc. nat.) \\ vorgelegt der \\ Mathematisch-naturwissenschaftlichen Fakultät \\ der \\ Universität Zürich \\ von \\ Anne Buttgereit \\ aus \\ Deutschland \\ Promotionskomitee \\ Prof. Dr. Melanie Greter \\ (Vorsitz und Leitung der Dissertation) \\ Prof. Dr. Burkhard Becher \\ Prof. Dr. Christian Münz \\ Prof. Dr. med. Frank Heppner
}

Zürich, 2017 



\section{Disclaimer}

This thesis is based on and adapted from the following publication:

Sall1 is a transcriptional regulator defining microglia identity and function

Anne Buttgereit, Iva Lelios, Xueyang Yu, Melissa Vrohlings, Natalie R Krakoski, Emmanuel L Gautier, Ryuichi Nishinakamura, Burkhard Becher \& Melanie Greter

Nature Immunology 17, 1397-1406 (2016), doi:10.1038/ni.3585 



\section{Table of contents}

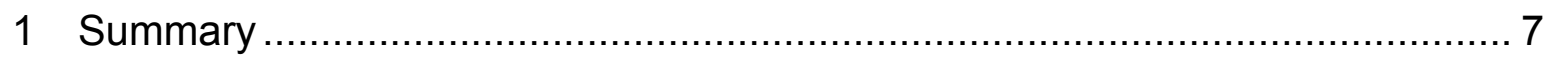

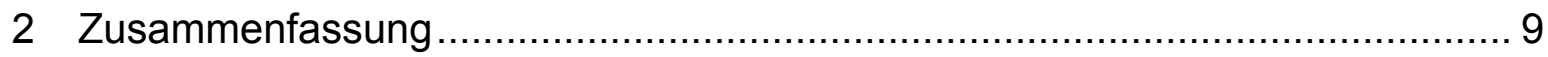

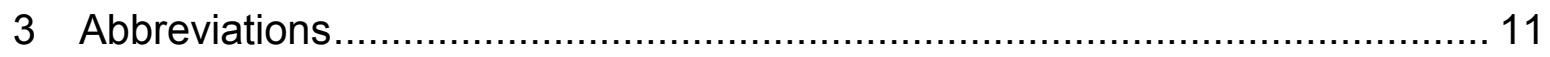

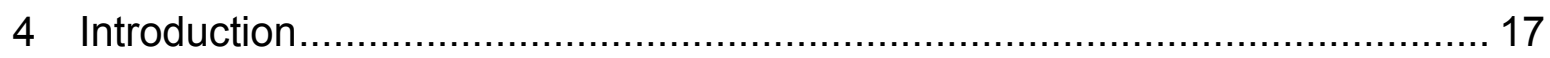

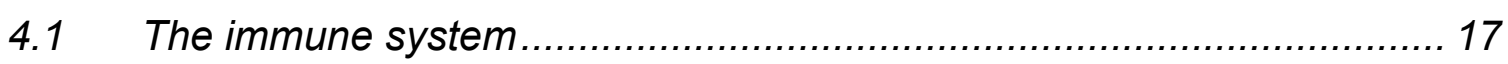

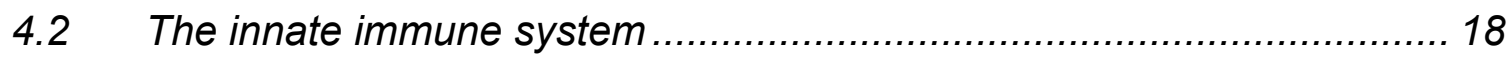

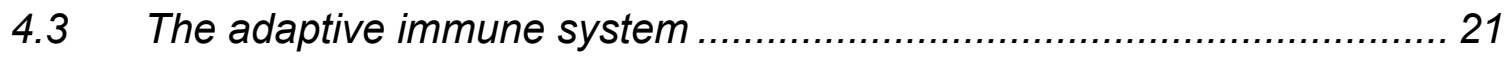

4.4 The mononuclear phagocyte system ……....................................... 24

4.5 Immune privilege in the central nervous system ................................. 30

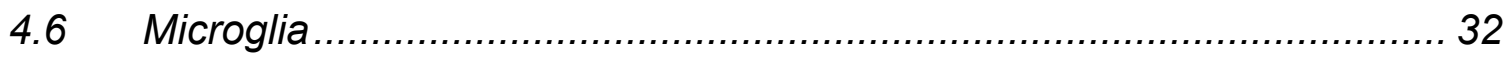

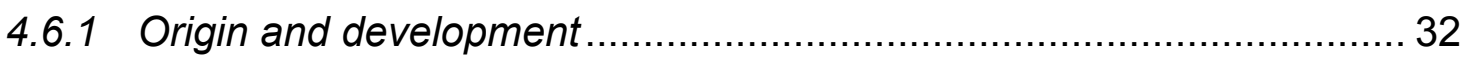

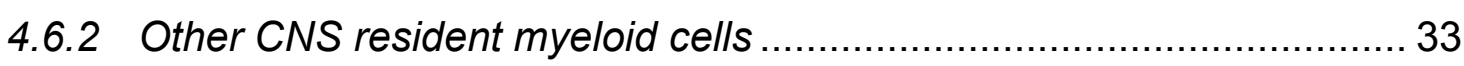

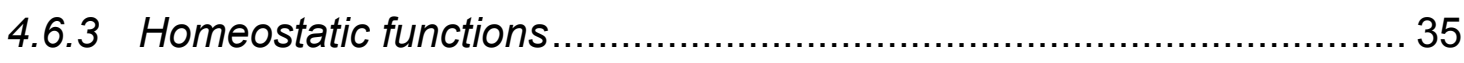

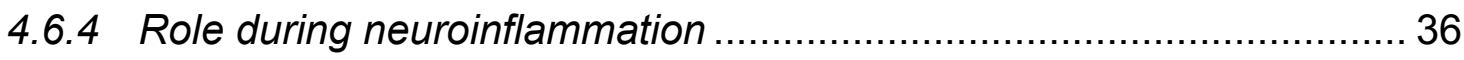

4.7 Transcription factors and genetic regulation ........................................ 39

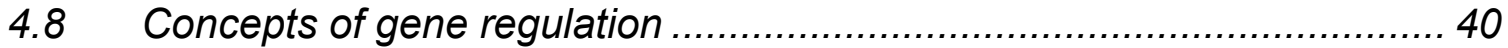

4.9 Zinc finger transcription factor Sall1 .............................................. 42

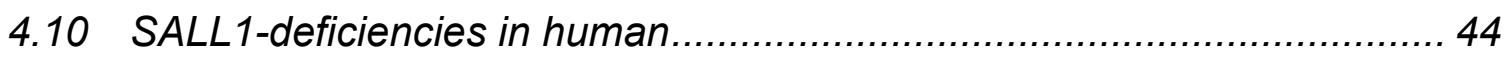

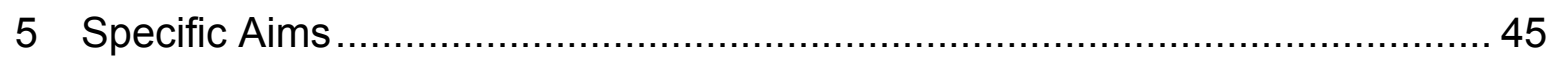

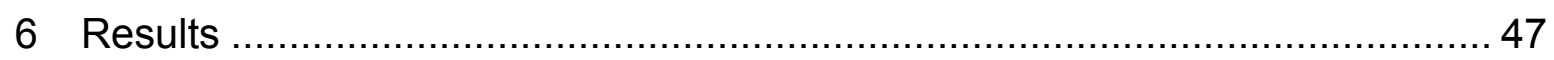

6.1 Microglia-specific expression of Sall1 ............................................. 47

6.2 The CNS environment alone does not imprint Sall1 expression............. 54

6.3 Csf1r and Tgfbr2 have different contributions to microglia homeostasis 59

6.4 Role of Sall1 and Tgfbr2 during microglia development ....................... 63

6.5 Sall1 controls the transcriptional signature of microglia .......................... 66

6.6 Sall1 regulates the microglia phenotype and function during tissue homeostasis and disease. 


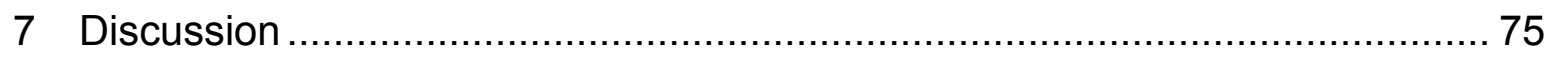

7.1 Identification of Sall1 as a transcription factor specific to microglia ........ 75

7.2 Targeting microglia in vivo using the Sall1 locus ............................... 76

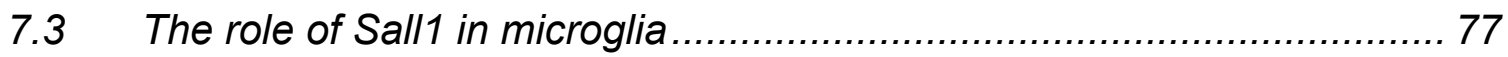

7.4 Microglial contributions to CNS damage and inflammation..................... 78

7.5 Translation of the Sall1 specificity to human ...................................... 79

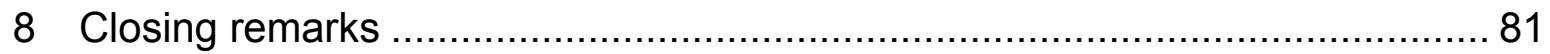

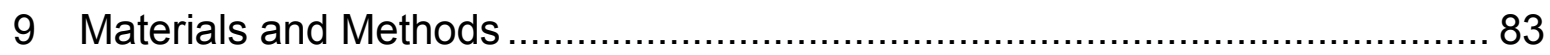

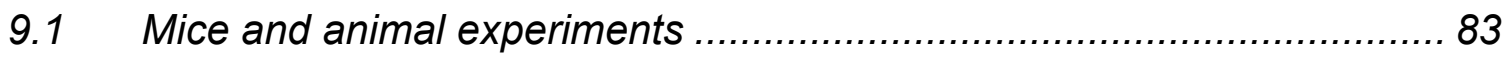

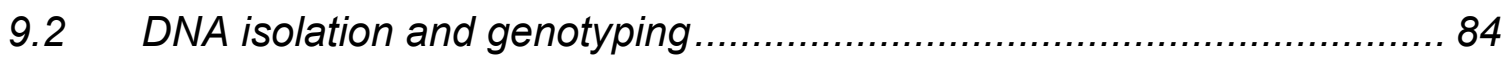

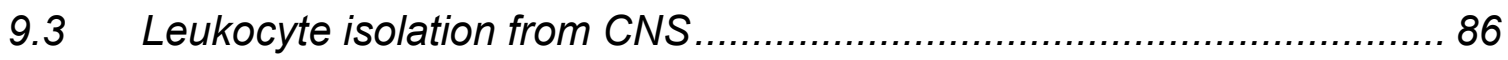

9.4 Leukocyte isolation from other organs .............................................. 86

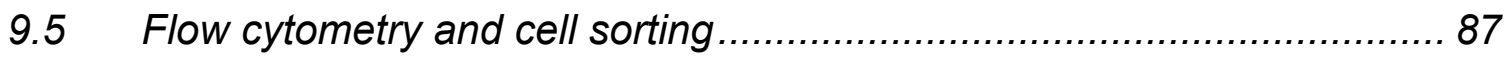

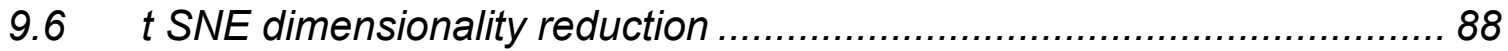

9.7 RNA isolation and quantitative rt-PCR …......................................... 88

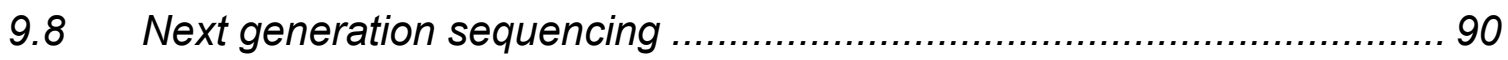

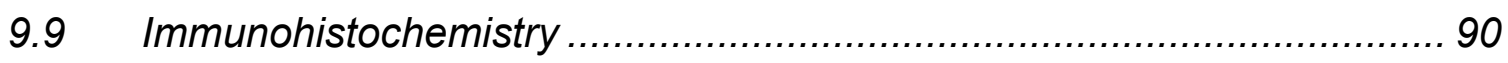

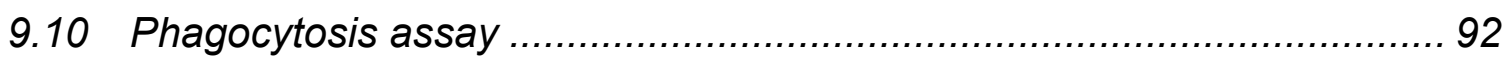

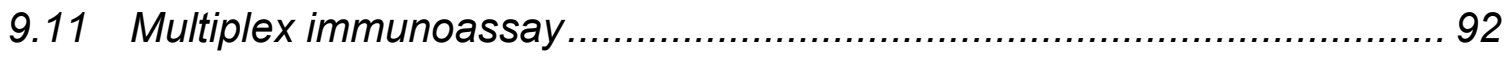

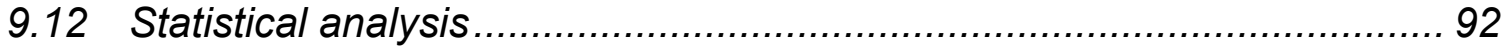

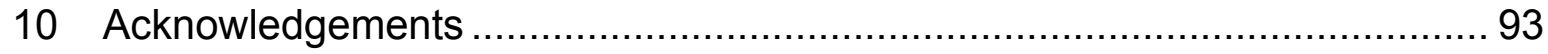

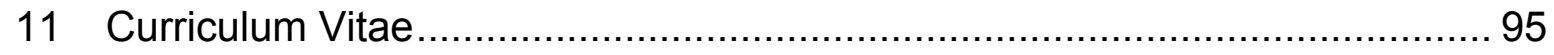

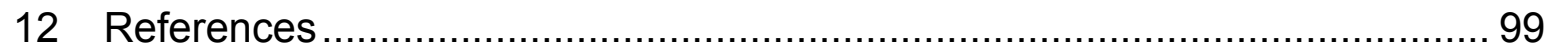




\section{Summary}

Microglia are the resident macrophages of the central nervous system (CNS). In contrast to other members of the mononuclear phagocyte system, including other tissue macrophages as well as monocytes and dendritic cells, microglia exclusively derive from extra-embryonic yolk sac precursors during early embryonic development. Under steady state conditions microglia are long-lived and selfmaintain locally without the contribution of bone marrow-derived monocytes. As the innate immune cells of the CNS microglia present the first line of defense against invading pathogens and other damaging insults. In addition to their immune functions, microglia also play an essential role in maintaining CNS homeostasis for example by clearing cellular debris and supporting the proper formation and function of the neuronal network. Despite extensive research, there is still little known about the contributions of microglia to CNS pathologies. Evidence so far suggests that microglia play a dual role in these setting being able to support regeneration as well as further tissue destruction.

In order to cope with the sensitive requirements of the CNS microglia harbor a unique genetic signature orchestrating their tissue-specific functions. Gene expression profiling has identified the transcriptional regulator Sall1 as one of the microglia signature genes. Sall1 is a zinc finger transcription factor originally identified in the proper organogenesis of limbs, kidneys, CNS and heart during embryonic development. Here we demonstrate that Sall1 is exclusively expressed by microglia within the hematopoietic system and the adult CNS. In contrast to previously described microglial markers, Sall1 is the first to specifically identify bona fide microglia and distinguish them from CNS-infiltrating monocyte-derived cells as well as from closely-related CNS-associated macrophages in the choroid plexus, meninges or perivascular areas. 
By exploiting the Sall1 locus for inducible gene targeting in adult mice, we achieved a specific and highly efficient recombination in microglia under steady state conditions. Using this novel tool, we established a role for macrophage colony stimulating factor receptor (Csf-1R) in the maintenance of adult microglia. Given the dependence of microglia on Csf-1R signaling for their development, the importance of this pathway during microglia maintenance has long been proposed but never conclusively been demonstrated due to the draw backs of previous models. In contrast to that, we did not observe a crucial role for transforming growth factor beta receptor (TGF- $\beta R$ ) in microglia survival during steady state. Our data rather indicate that signaling via TGF- $\beta R$ is important to suppress excessive activation of microglia. We further utilized the microglia-specific expression of Sall1 to inducibly inactivate the Sall1 locus in vivo. This resulted in the transformation of microglia from 'resting' tissue macrophages into inflammatory phagocytes leading to disturbed tissue homeostasis and altered neurogenesis. Collectively, Sall1 transcriptional regulation maintains microglia identity and physiological properties in the CNS, and offers a novel route to microglia-specific manipulations in vivo. 


\section{Zusammenfassung}

Mikroglia sind die lokalen Makrophagen des zentralen Nervensystems (ZNS) und somit gleichzeitig Bestandteil des mononukleären Phagozyten-Systems (MPS), welches sämtliche Gewebsmakrophagen sowie Monozyten und dendritischen Zellen umfasst. Im Gegensatz zu anderen Mitgliedern des MPS entstehen Mikroglia ausschließlich während der frühen Embryonalentwicklung aus extraembryonalen Dottersack-Vorläuferzellen. Unter homöostatischen Bedingungen sind Mikroglia langlebige Zellen, die sich lokal selbst regenerieren ohne den Einfluss von Monozyten aus dem Knochenmark. Mikroglia sind die angeborenen Immunzellen des ZNS und bilden die erste Verteidigungslinie gegen eindringende Krankheitserreger und andere schädliche Einflüsse. Zusätzlich zu ihren Immunfunktionen sind Mikroglia auch für die Aufrechterhaltung der ZNSHomöostase wichtig. Sie entfernen beispielsweise Überreste von abgestorbenen Zellen und tragen zur korrekten Bildung und Funktion des neuronalen Netzwerkes bei. Über die genauen Funktionen von Mikroglia bei verschiedenen Erkrankungen des ZNS ist trotz intensiver Forschung bisher nur bekannt. Man geht jedoch davon aus, dass sie eine duale Rolle spielen, die sowohl Regeneration als auch fortschreitende Schädigung des ZNS unterstützen kann.

Um den sensiblen Anforderungen des ZNS gerecht zu werden, haben Mikroglia eine spezifische genetische Signatur, die es innen ermöglicht ihre gewebespezifischen Funktionen auszuüben. Die systematische Analyse von Genexpressionsprofilen hat ergeben, dass eines dieser mikroglia-spezifischen Gene der Transkriptionsregulator Sall1 ist. Sall1 ist ein so genannter Zink-FingerTranskriptionsfaktor, der ursprünglich während der Embryonalentwicklung im Zusammenhang mit der vollständigen Entwicklung von Gliedmaßen, Nieren, ZNS und Herz identifiziert wurde. Wir konnten in unserer Studie zeigen, dass Sall1 innerhalb des hämatopoetischen Systems und des adulten ZNS ausschließlich von Mikroglia exprimiert wird. Im Gegensatz zu bisher beschriebenen MikrogliaMarkern ist Sall1 der erste, der spezifisch für embryonal-entstandene Mikroglia ist. Er erlaubt ihre Unterscheidung sowohl von eingewanderten Monozyten aus dem Knochenmark, und draus hervorgehenden Zellen, als auch von den eng verwandten ZNS-assoziierten Makrophagen im Plexus choroideus, in den Hirnhäuten und in perivaskulären Bereichen. 
Durch die Nutzung des Sall1-Lokus' für induzierbare Genmanipulationen in erwachsenen Mäusen konnten wir Mikroglia spezifisch und effizient verändern. Mit Hilfe dieses neuen Systems konnten wir dem Zytokinrezeptor Csf-1R eine wichtige Funktion im Erhalt von Mikroglia während der Homöostase zuteilen. Nachdem bereits gezeigt wurde, dass die Entwicklung von Mikroglia von diesem Rezeptor abhängt, lag die Vermutung nahe, dass dieser auch für die Erhaltung der Zellen wichtig ist. Dies konnte bisher jedoch nicht abschießend gezeigt werden. Auch einem weiteren Zytokinrezeptor, TGF- $\beta R$, wurde eine wichtige Rolle in der Entwicklung von Mikroglia zugesprochen. Im Gegensatz zu Csf-1R, konnten wir für TGF- $\beta R$ jedoch keine entscheidende Rolle im Erhalt von Mikroglia während der Homöostase beobachten. Unsere Daten zeigen jedoch, dass die Signalweiterleitung über TGF- $\beta R$ wichtig ist, um eine übermäßige Aktivierung von Mikroglia zu unterdrücken. Darüber hinaus haben wir die Mikroglia-spezifische Expression von Sall1 verwendet, um den Sall1-Lokus in erwachsenen Mäusen zu inaktivieren. Diese Inaktivierung führte dazu, dass die „ruhenden“ Gewebsmakrophagen zu entzündlichen Phagozyten transformierten, was in einer gestörten Gewebshomöostase und in Veränderungen der Neurogenese resultierte. Zusammenfassend lässt sich sagen, dass die genetische Regulation von Mikroglia durch Sall1 die Identität und physiologischen Eigenschaften dieser wichtigen Zellen im ZNS bestimmt und damit einen neuen Weg bietet, Mikroglia spezifisch zu manipulieren. 


\section{Abbreviations}

\begin{tabular}{|c|c|}
\hline AA & amino acid \\
\hline Ac & acetylation \\
\hline$A D$ & Alzheimer's disease \\
\hline Ahr & aryl hydrocarbon receptor \\
\hline AIM2 & absent in melanoma 2 \\
\hline AIRE & autoimmune regulator gene \\
\hline ALR & AIM2-like receptors \\
\hline ALS & amyotrophic lateral sclerosis \\
\hline AM & alveolar macrophages \\
\hline AMG & aorta-gonad-mesonephros \\
\hline APC & antigen presenting cells \\
\hline ATP & adenosine triphosphate \\
\hline Axl & Tyrosine-protein kinase receptor UFO \\
\hline$A \beta$ & amyloid beta \\
\hline BBB & blood brain barrier \\
\hline BCR & B cell receptor \\
\hline BDNF & brain-derived neurotrophic factor \\
\hline BM & bone marrow \\
\hline C57BL/6 & inbred mouse strain \\
\hline $\mathrm{Ca}$ & calcium \\
\hline CCL2 & CC-chemokine ligand 2 \\
\hline$C D$ & cluster of differentiation \\
\hline $\mathrm{cDC}$ & classical or conventional DC \\
\hline cDNA & complementary DNA \\
\hline CFA & Complete Freund's Adjuvant \\
\hline CLR & C-type lectin receptors \\
\hline cMoP & common monocyte progenitor \\
\hline CNS & central nervous system \\
\hline CNS-MF & CNS-associated macrophages \\
\hline $\mathrm{CO}_{2}$ & Carbon dioxide \\
\hline $\mathrm{CP}$ & choroid plexus \\
\hline CpG & 5'-C-phosphate-G-3' \\
\hline CreER & tamoxifen-inducible Cre recombinase \\
\hline CSF & cerebrospinal fluid \\
\hline Csf & colony-stimulating factor \\
\hline Csf-1/ M-Csf & macrophage colony stimulating factor \\
\hline Csf-1R & macrophage colony stimulating factor receptor \\
\hline
\end{tabular}

Csf-2/ GM-Csf granulocyte-macrophage colony stimulating factor 


\begin{tabular}{|c|c|}
\hline $\mathrm{CX}_{3} \mathrm{CL} 1$ & fractalkine \\
\hline $\mathrm{CX}_{3} \mathrm{CR} 1$ & fractalkine receptor \\
\hline Cxcl10 & Interferon gamma-induced protein 10 (IP-10) \\
\hline Cybb & cytochrome b subunit beta / NADPH oxidase 2 \\
\hline Cyp4f18 & cytochrome P450, family 4, subfamily f, polypeptide 18 \\
\hline $\mathrm{D}$ & diversity gene \\
\hline DAMP & damage-associated molecular pattern \\
\hline DAP12 & DNAX-activating protein of $12 \mathrm{kDa}$ \\
\hline DC & dendritic cells \\
\hline DCX & doublecortin \\
\hline $\mathrm{ddH}_{2} \mathrm{O}$ & double-distilled water \\
\hline DNA & deoxyribonucleic acid \\
\hline dNTP & deoxy-nucleoside triphosphate \\
\hline dpp & decapentaplegic \\
\hline DTT & Dithiothreitol \\
\hline$E$ & embryonic day \\
\hline EAE & experimental autoimmune encephalomyelitis \\
\hline EDTA & Ethylenediaminetetraacetic acid \\
\hline EMP & erythro-myeloid progenitors \\
\hline EpCam & Epithelial cell adhesion molecule \\
\hline $\mathrm{F} 4 / 80$ & EGF-like module-containing mucin-like hormone receptor-like 1 \\
\hline FACS & Fluorescence-activated cell sorting \\
\hline FCS & fetal calf serum \\
\hline FLT3 & FMS-like tyrosine kinase 3 \\
\hline FLT3L & FMS-like tyrosine kinase 3 ligand \\
\hline FMS & Feline McDonough Sarcoma \\
\hline Foxp3 & forkhead box protein 3 \\
\hline G & gauge (needle thickness) \\
\hline GFAP & Glial fibrillary acidic protein \\
\hline GFP & green fluorescent protein \\
\hline GLAST & glutamate/ aspartate transporter \\
\hline H3K27Ac & histone H3 lysine 27 acetylation \\
\hline H3K36me3 & histone H3 lysine 36 trimethylation \\
\hline H3K4me3 & histone H3 lysine 4 trimethylation \\
\hline H3K79me2 & histone H3 lysine 79 dimethylation \\
\hline HBSS & Hanks' Balanced Salt Solution \\
\hline HSC & hematopoietic stem cells \\
\hline I-A/I-E & murine $\mathrm{MHC}$ class two \\
\hline i.p & intra peritoneal \\
\hline lba-1 & ionized calcium-binding adapter molecule 1 \\
\hline
\end{tabular}




\begin{tabular}{|c|c|}
\hline iDTR & inducible Diphtheria Toxin Receptor \\
\hline Ifit & Interferon Induced proteins with Tetratricopeptide repeats \\
\hline Ifitm3 & Interferon Induced Transmembrane Protein 3 \\
\hline IFN & interferons \\
\hline $\lg$ & immunoglobulin \\
\hline $\operatorname{lgf1}$ & Insulin-like growth factor 1 \\
\hline $\mathrm{IHC}$ & immunohistochemistry \\
\hline $\mathrm{IL}$ & interleukins \\
\hline ILC & innate lymphoid cells \\
\hline Irf & Interferon Regulatory Factor \\
\hline Itgal & integrin alpha $L$ chain \\
\hline iTregs & adaptive or induced Tregs \\
\hline J & joining gene \\
\hline $\mathrm{kb}$ & kilo base pairs \\
\hline kDa & kilo Dalton (protein size unit) \\
\hline $\mathrm{KHCO}_{3}$ & Potassium bicarbonate \\
\hline LC & Langerhans cells \\
\hline LPS & lipopolysaccharide \\
\hline LRR & leucine-rich repeat \\
\hline $\mathrm{m}$ & meningeal \\
\hline M-MLV & reverse transcriptase \\
\hline $\mathrm{mAb}$ & monoclonal antibodies \\
\hline MAC & membrane attack complex \\
\hline MALT & mucosa-associated lymphoid tissues \\
\hline MAPK & Mitogen-activated protein kinases \\
\hline MBL & mannose binding lectin \\
\hline MBP & Myelin basic protein \\
\hline MC & monocyte-derived cells \\
\hline MDA5/ IFIH1 & melanoma differentiation-associated protein 5 \\
\hline $\mathrm{Me}$ & methylation \\
\hline MerTK & MER Proto-Oncogene Tyrosine Kinase \\
\hline MF & macrophages \\
\hline $\mathrm{Mg}$ & magnesium \\
\hline $\mathrm{MHC}$ & major histocompatibility complex \\
\hline MoDC & monocyte-derived dendritic cells \\
\hline MOG & Myelin Oligodendrocyte Glycoprotein \\
\hline MPS & mononuclear phagocyte system \\
\hline mRNA & messenger RNA \\
\hline MS & multiple sclerosis \\
\hline Msr1 & Macrophage Scavenger Receptor 1 \\
\hline
\end{tabular}




\begin{tabular}{|c|c|}
\hline Mx1 & Myxovirus Resistance 1 \\
\hline $\mathrm{NaOH}$ & sodium hydroxid \\
\hline ncRNA & noncoding RNA \\
\hline NeuN & Hexaribonucleotide Binding Protein-3 \\
\hline NF-KB & nuclear factor $\mathrm{kB}$ \\
\hline NGS & next generation sequencing \\
\hline $\mathrm{NH}_{4} \mathrm{Cl}$ & ammonium chloride \\
\hline NK & natural killer \\
\hline NLR & NOD-like receptors \\
\hline NLRP & Nod-like receptor protein \\
\hline NO & nitric oxide \\
\hline NOD & nucleotide-binding oligomerization domain \\
\hline NP & neutrophils \\
\hline nTregs & natural Tregs \\
\hline NuRD & nucleosome remodeling and deacetylase \\
\hline o.g. & oral gavage \\
\hline Olig2 & oligodendrocyte transcription factor 2 \\
\hline$P$ & phosphorylation \\
\hline P2ry12 & purinergic receptor P2Y, G-protein coupled 12 \\
\hline PAMP & pathogen-associated molecular patterns \\
\hline PAP & pulmonary alveolar proteinosis \\
\hline PBS & phosphate buffered saline \\
\hline PCR & polymerase chain reaction \\
\hline pDCs & plasmacytoid DCs \\
\hline PDGFR & Platelet-derived growth factor receptor \\
\hline pHrodo & pH-sensitive red dye \\
\hline PLO & primary lymphoid organs \\
\hline PLX5622 & Csf-1R inhibitor (Plexxikon) \\
\hline Pol II & RNA polymerase two \\
\hline PPARy & peroxisome proliferator-activated receptor gamma \\
\hline PPR & pattern recognition receptors \\
\hline ppt & parts-per-trillion, $10^{-12}$ \\
\hline pv & perivascular \\
\hline PYD & pyrin domain \\
\hline Q & amino acid glutamine \\
\hline qPCR & quantitative real-time PCR \\
\hline $\mathrm{R} 26$ & Rosa26 locus \\
\hline RA & retinoic acid \\
\hline RAG & recombinase-activating gene \\
\hline RIG-I & retinoic acid-inducible gene I \\
\hline
\end{tabular}




\begin{tabular}{|c|c|}
\hline RLR & RIG-I-like receptors \\
\hline RNA & ribonucleic acid \\
\hline RNA-seq & RNA-sequencing \\
\hline ROS & reactive oxygen species \\
\hline rpm & rotations per minute \\
\hline RT & room temperature \\
\hline Runx1 & Runt Related Transcription Factor 1 \\
\hline S100B & S100 calcium-binding protein B \\
\hline Sall & spalt like gene \\
\hline SAS & subarachnoid space \\
\hline Serpinf1 & Pigment epithelium-derived factor \\
\hline SGZ & subgranular zone \\
\hline Siglec & Sialic acid binding Ig-like lectin \\
\hline Slc2a5 & Solute Carrier Family 2 Member 5 \\
\hline SLO & secondary lymphoid organs \\
\hline Stard13 & StAR-related lipid transfer domain protein 13 \\
\hline $\mathrm{Su}$ & sumoylation \\
\hline t-SNE & t-Distributed Stochastic Neighbor Embedding \\
\hline TBS & Townes-Brocks syndrome \\
\hline TCR & T cell receptor \\
\hline TdT & Terminal deoxynucleotidyl transferase \\
\hline TF & transcription factor \\
\hline TGF- $\beta$ & Transforming growth factor beta \\
\hline TGF- $\beta R$ & Transforming growth factor beta receptor \\
\hline TLR & Toll-like receptors \\
\hline TNFa & Tumor necrosis factor alpha \\
\hline Tregs & regulatory $\mathrm{T}$ cells \\
\hline TREM2 & Triggering receptor expressed on myeloid cells 2 \\
\hline TrisHCl & Tris-Hydrochloride \\
\hline $\mathrm{Ub}$ & ubiquitination \\
\hline V & variable \\
\hline Vav1 & Vav Guanine Nucleotide Exchange Factor 1 \\
\hline Vcam1 & Vascular cell adhesion molecule 1 \\
\hline WT & wild type \\
\hline YFP & yellow fluorescent protein \\
\hline YS & yolk sac \\
\hline ZNS & zentrales Nervensystem \\
\hline
\end{tabular}


Abbreviations 


\section{Introduction}

\subsection{The immune system}

The mammalian immune system plays a central role in defending the host against various pathogens including bacteria, viruses and other parasites as well as harmful or toxic substances like allergens or venoms. It is divided into two arms namely the innate and the adaptive immune system, both of which have humoral and cellular components (Figure 1). Initially it has been thought that the innate and adaptive immune system act successive and independently of each other, however recent evidence shows a strong complementation and cooperation between the two branches of the immune system ${ }^{1}$.

In order to fully develop and function the immune system needs specialized structures, designated lymphoid tissues. These tissue structures can be divided into primary lymphoid organs (PLO), namely bone marrow (BM) and thymus, important for the development and maturation of immune cells and secondary lymphoid organs (SLO), including spleen, lymph nodes, tonsils, Peyer's Patches and mucosa-associated lymphoid tissues (MALT). The main functions of SLO are to reduce the pathogen spread by strategically localized phagocytes, to facilitate effective interactions between different players of the immune system by bringing them in close proximity and to provide necessary factors for the differentiation and survival of lymphocytes. Furthermore, ectopic lymphoid aggregates at the site of infection can function as tertiary lymphoid organs harboring defined immune structures and memory cells ${ }^{2}$.

In addition to their role in immune protection, elements of the immune system also play key roles in maintaining normal tissue homeostasis. 


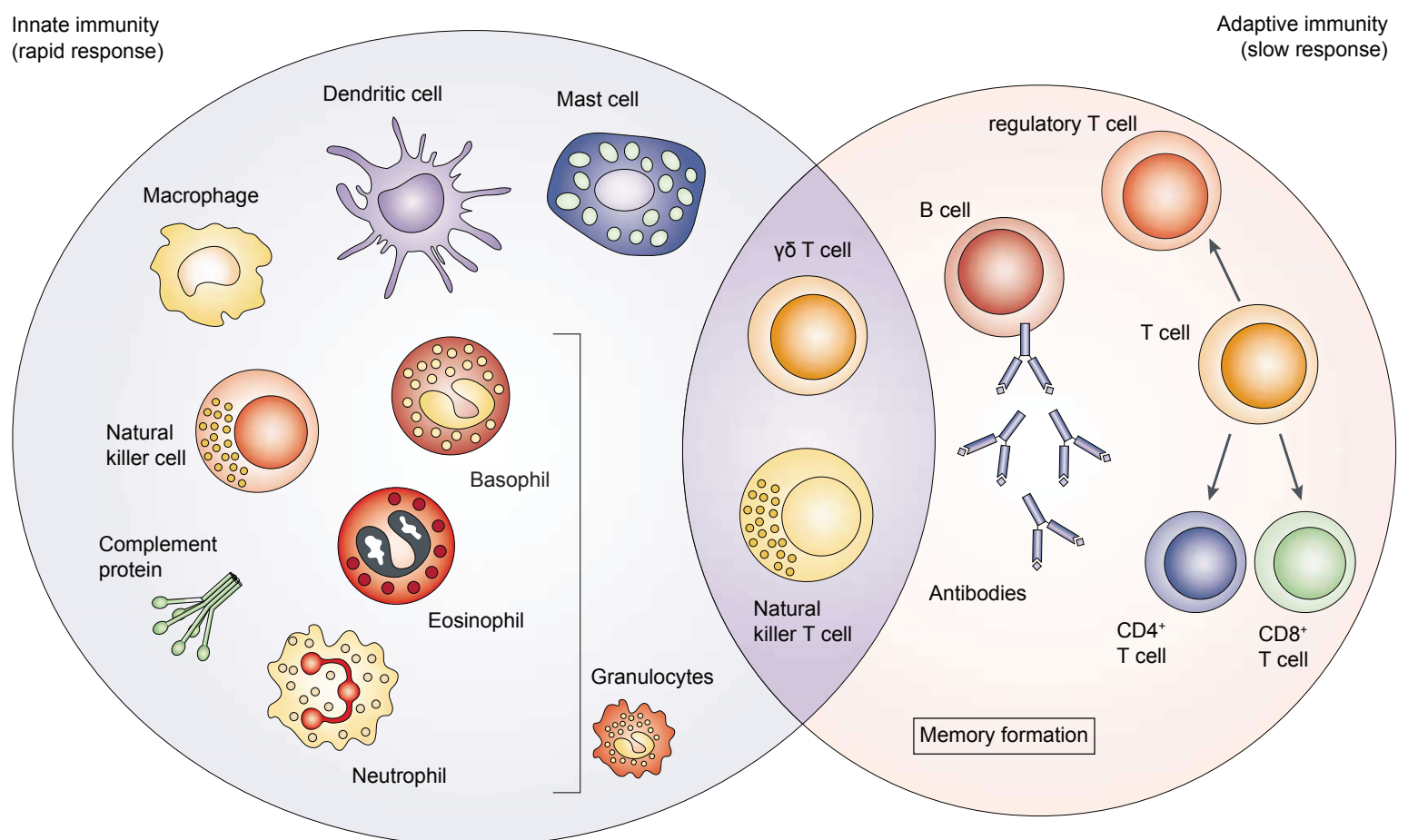

Figure 1 | The innate and adaptive immune response. The innate immune response functions as the first line of defense against infection. It consists of soluble factors, such as complement proteins, and diverse cellular components including granulocytes (basophils, eosinophils and neutrophils), mast cells, macrophages, dendritic cells and natural killer cells. The adaptive immune response is slower to develop, but manifests as increased antigenic specificity and memory. It consists of B cells, different $T$ cells subsets including CD4+ and CD8+ $T$ cells as well as regulatory $T$ cells and antibodies as humoral components. In addition, natural killer $T$ cells and $y \delta T$ cells are cytotoxic lymphocytes that straddle the interface of innate and adaptive immunity (adapted from ${ }^{3}$ ).

\subsection{The innate immune system}

The innate immune system is comprised of physical, chemical and microbiological barriers, including epidermal surfaces, mucus or cilia that prevent pathogens from entering the body. In addition it harbors cellular effectors mainly of the myeloid lineage and serum components like the complement system and acute phase proteins. The innate immune system is highly conserved across different species and is characterized by a rapid response towards pathogenic agents based on the recognition of specific patterns ${ }^{4}$.

Innate immune cells are strategically positioned in many tissues and exert crucial functions in the initial response towards pathogenic insults and tissue injury ${ }^{5}$. They are comprised by a multitude of cells from both the lymphoid lineage, like natural killer (NK) cells and innate lymphoid cells (ILC) as well as the myeloid lineage, including monocytes, macrophages (MF), dendritic cells (DC) and granulocytes 
such as neutrophils (NP), basophils and eosinophils (Figure 1) ${ }^{3}$. NK cells are armed with potent cytotoxic effector functions that are initiated based on integration of signals from activating and inhibitory receptors ${ }^{6}$. Recognition of self major histocompatibility complex (MHC) class $\mathrm{I}$ is one of the main inhibitory signals, which makes NK cells potent killers of cells that down-regulate self-MHC-I due to viral infections or tumorigenic modifications ${ }^{1}$. Neutrophils, macrophages and monocytes are the major phagocytic cells of the innate immune system, they engulf pathogenic microbes and destroy them in vesicles called phagolysosoms by toxic effector molecules, such as nitric oxide (NO), superoxide, and degradative enzymes. This process can be enhanced significantly by the opsonisation of the particles with complement molecules or specific antigens ${ }^{1,4}$. With the uptake and processing of microbial antigens specialized antigen presenting cells (APC) like DCs, play a key role in adaptive immunity by presenting processed antigen peptides to $\mathrm{T}$ cells in a context of $\mathrm{MHC}$ molecules ${ }^{1}$. Additionally innate immune cells modulate innate and adaptive immune responses by their secretion of specific immune-modulatory cytokines, like interleukins (IL), chemokines, colonystimulating factors (Csf) or interferons (IFN).

As mentioned before innate immune cells are able to discriminate invading pathogens and damaged cells from commensal microorganisms and normal self using so-called pattern recognition receptors (PPR). Their recognition is based on conserved pathogen-associated molecular patterns (PAMP) or damage-associated molecular pattern (DAMP) respectively and is crucial for host defense and tissue remodeling ${ }^{7}$. There are three main classes of PPRs, namely Toll-like receptors (TLR), RIG-I-like receptors (RLR) and NOD-like receptors (NLR), as well as C-type lectin receptors (CLR), AIM2-like receptors (ALR) and a family of enzymes functioning as intracellular sensors of nucleic acids all together covering the broad variety of molecular patterns ${ }^{8}$. TLRs are expressed on the plasma membrane of innate immune cells and are able to recognize a wide range of extracellular pathogens. TLR1, TLR2, TLR4, TLR5, TLR6 and TLR11 are specialized to recognize components of bacterial and fungal cell walls, such as lipopolysaccharides (LPS), bacterial lipopeptides, flagellin or glycolipids. TLR3, TLR7, TLR8, TLR9 and TLR13 on the other hand are specialized on the recognition of viral nucleic acids in endosomal compartments such as CpG-DNA or 
free single- or double-stranded RNA ${ }^{9}$. Upon ligation of PAMPs to TLRs a signaling cascade centered around the nuclear factor $K B(N F-K B)$ is initiated leading to the expression of pro-inflammatory cytokines and type I IFN ${ }^{7}$. RLRs are a family of cytoplasmic RNA helicases important for the recognition and control of viruses including their replication and dissemination. Among those PPRs is the eponymous retinoic acid-inducible gene I (RIG-I) as wells as the melanoma differentiationassociated protein 5 (MDA5; also known as IFIH1). Engagement of RLRs also results in the production of type I IFN ${ }^{7}$. The third main group of PPRs, NLRs, are essential for host defense against bacterial infections. They are comprised of a large group of intracellular proteins including the nucleotide-binding oligomerization domain proteins (NODs) and NOD-, leucine-rich repeat (LRR) and pyrin domain (PYD)- containing proteins (NLRP) that recognize peptidoglycan motifs from bacterial cells, bacterial toxins or stress induced danger molecules like extracellular adenosine triphosphate ATP and uric acid crystals. NLR signaling also leads to the activation of NF-KB and Mitogen-activated protein kinases (MAPK) resulting in the production of pro-inflammatory cytokines. Furthermore by the activation of Caspase-1 NLRs are involved in the formation of so called inflammasomes inducing pro-inflammatory cascades ${ }^{10}$. Even though the immune response initiated by PPRs is crucial for immediate host defense and the instruction of the adaptive immune system it needs to be tightly regulated in order to avoid excessive tissue damage and immunopathology. The regulatory network includes a multitude of enzymes, transcription factors and epigenetic molecules modulating distinct steps of the PPR signaling cascade in a cooperative or antagonistic manner ${ }^{7}$.

Apart from cell mediated responses serum factors also play a central role in innate immune reactions. Among those the complement system is the most prominent player comprising a complex network of more than 30 plasma and membraneassociated serum proteins that induce highly efficient inflammatory and cytolytic immune responses ${ }^{11}$. The complement proteins are organized in a hierarchy of proteolytic and assembly cascades that mount in the formation of a C3 convertase, cleaving the central complement component $\mathrm{C} 3$ into its active subunits (C3a, C3b). There are three types of complement activation pathways, namely the classical, the lectin and the alternative pathway depending on the initiating factor. While the classical pathway is activated by antigen-antibody complexes of the adaptive 
immune response, the other two pathways are initiated by means of the innate immune defense. The lectin pathway hereby relies on the recognition of carbohydrates by PPRs like mannose binding lectin (MBL) or ficolins, whereas the alternative pathways is activated independently of PPRs by direct binding of $\mathrm{C} 3$ to hydroxyl or amine groups on the cell surface of microorganisms ${ }^{12}$. Regardless of the initiation pathway, activation of the complement system converges to three main effector pathways. These include direct lysis of targeted surfaces by the assembly of the membrane attack complex (MAC), alerting and priming of the immune system by means of pro-inflammatory anaphylatoxins (C3a, C5a), and opsonization and clearance of target surfaces through recognition of complement opsonins (C4b, C3b, C3bi) by complement receptors on phagocytic cells, such as macrophages and neutrophils ${ }^{11}$.

\subsection{The adaptive immune system}

The adaptive immune system is specific to higher animals and is characterized by a slower but much more specific immune response and the ability to form a longlived immune memory (Figure 1). Adaptive immunity is primarily based on the use of clonally diverse antigen-specific receptors on B and T cells. These receptors are encoded by genes that assemble through somatic DNA rearrangement and random chain pairing to permit the formation of millions of different antigen receptors ${ }^{1}$. The effector response is divided into two steps, first the priming, activation and differentiation of the respective $B$ or $T$ cells after engagement with its specific antigen, which usually occurs within lymphoid tissues. And second, the effects that are mediated by the extravasation of activated $T$ cells to the site of infection or the release of antibodies from activated B cells, so called plasma cells, into the blood and lymph system ${ }^{4}$.

T cells develop from a common lymphoid progenitor in the bone marrow that migrates to the thymus for further differentiation, thus the name $T$ cell. There are two main classes of T cells, namely $\alpha \beta$ and $\gamma \delta T$ cells, depending on the $T$ cell receptor (TCR) chains that are used, with the vast majority bearing an $\alpha \beta$ TCR. Within specialized compartments of the thymus the individual chains of the TCR are assembled from variable $(\mathrm{V})$, diversity $(\mathrm{D})$ and joining $(\mathrm{J})$ gene elements by somatic rearrangement to generate mature $V J \alpha$-chains and VDJ $\beta$-chains. The 
assembly of these gene elements is initiated by the lymphoid-specific recombinase-activating gene (RAG) 1 and RAG 2 proteins, and further diversity is introduced by the insertion additional nucleotides into some of the VDJ junctions by the enzyme terminal deoxynucleotidyl transferase (TdT) ${ }^{1}$. The TCR forms a complex with the co-signaling molecule $\mathrm{CD} 3$ and the thymocytes start to express the co-receptors $\mathrm{CD} 8$ and $\mathrm{CD} 4{ }^{13}$. The $\mathrm{CD} 4^{+} \mathrm{CD} 8^{+}$double-positive cells undergo positive selection in the thymic cortex to assure sufficient affinity of the TCR to selfMHC molecules. During this step the immature T cells become single-positive in accordance with the MHC class they were selected on, namely $\mathrm{CD} 4^{+}$cells are restricted to antigen recognition via MHC-II while $\mathrm{CD} 8^{+}$cells are restricted to $\mathrm{MHC}$ I related antigen presentation. Another important regulatory step during $\mathrm{T}$ cell development is the negative selection in the thymic medulla, where pre-mature $T$ cells are screened for auto-reactivity to tissue specific self-proteins under the control of the autoimmune regulator gene (AIRE) ${ }^{1}$. More than $95 \%$ of immature T cells undergo apoptosis during thymic selection due to low MHC-affinity or autoreactivity. Both $\mathrm{T}$ cell linages exert distinct effector function, in general $\mathrm{CD} 4^{+} \mathrm{T}$ cells are designated $\mathrm{T}$ helper cells that activate both humoral and cellular immune responses by the production of cytokines, whereas $\mathrm{CD} 8^{+} \mathrm{T}$ cells possess cytotoxic activities against infected or tumorigenic cells ${ }^{4}$. In addition to effector $T$ cells a portion of $\mathrm{CD}^{+}{ }^{+} \mathrm{T}$ cells are designated regulatory $\mathrm{T}$ cells (Tregs) that downregulate immune responses and maintain systemic tolerance. Tregs are characterized by the expression of the transcription factor (TF) forkhead box protein 3 (Foxp3), which is important for their development, and the surface marker CD25 (IL-2R). Two subsets of Tregs can be distinguished, namely the natural Tregs (nTregs), that develop their regulatory phenotype already in the thymus, while the adaptive or induced Tregs (iTregs) only differentiate in the periphery from naïve $\mathrm{CD} 4^{+} \mathrm{T}$ cells in the presence of IL-10, transforming growth factor $\beta$ (TGF- $\beta$ ) or retinoic acid $(\mathrm{RA})^{14}$. 
B cells are bone-marrow derived lymphocytes that are defined by their production of immunoglobulin ( $\mathrm{lg}$ ), which make up the $B$ cell receptor $(B C R)$ in a membranebound form and are secreted as antibodies in a soluble form. Ig molecules are composed of two identical heavy ( $50 \mathrm{kDa})$ and light $(25 \mathrm{kDa})$ chain pairs resulting in the formation of two identical antigen-binding site on each molecule. The assembly of the individual heavy and light chains occurs similar to that of TCRs by the somatic rearrangement of VJ (light chain) or VDJ (heavy chain) gene elements ${ }^{1}$. The variable region of each heavy and light chain pair is located at the aminoterminal portion of the $\mathrm{lg}$ molecule and creates the antigen-binding site. Each variable region includes 3 hypervariable sequences that allow for somatic mutations in the process of affinity maturation and clonal expansion of $B$ cells in the germinal centers of secondary lymphoid tissues after immune activation ${ }^{15}$. The carboxy-terminal portion of the heavy and light chains is constant within each antibody subclass. In line with that, the constant regions of the heavy chains form the $\mathrm{Fc}$ domain of the $\mathrm{lg}$ molecule that exerts most of the effector functions, including Fc receptor engagement and complement activation ${ }^{1}$. In a naïve state $B$ cells only express $\lg \mathrm{M}$ and $\lg \mathrm{D}$ molecules. However upon immune activation, under the influence of helper $T$ cells, isotype switching occurs by means of alternative splicing leading to a change in the constant region of the $\mathrm{lg}$ molecule while the previously rearranged variable region and thereby the antigen specificity remains unchanged. This allows the $B$ cell to produce antibodies with the same antigenic specificity but different effector functions ${ }^{15}$.

Both $B$ and $T$ lymphocytes are able to form long-lived memory cells that reside within the tissues and are able to mount a much faster adaptive immune response upon re-encounter of a known pathogen. 


\subsection{The mononuclear phagocyte system}

The mononuclear phagocyte system (MPS) represents one of the main cellular components of the innate immune system, including monocytes, macrophages and dendritic cells. Historically the MPS has been classified as a pool of functionally related myeloid cells that derive from a common progenitor in the bone marrow and are constantly replenished ${ }^{16}$. This traditional view has been largely revised today appreciating ontogenic and functional differences between the individual cell subsets. Recent efforts have been made to imply a uniform nomenclature based on two levels, first the cellular origin and second the function, location and/or morphology (Figure 2) ${ }^{17}$. A clear separation however remains challenging due to historical views, large heterogeneity within each population and plasticity among subpopulations.
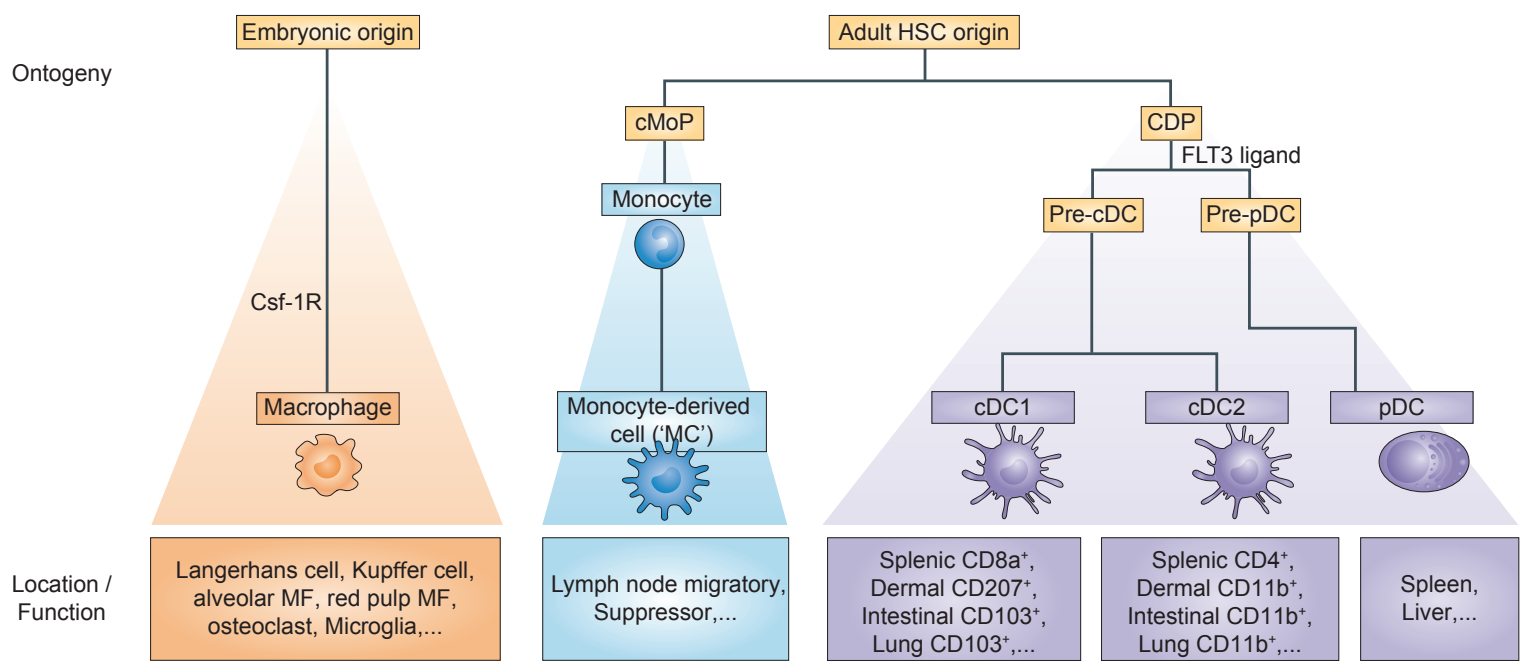

Figure 2 | Classification of mononuclear phagocytes. Mononuclear phagocytes can be defined on the basis of their ontogeny (level one nomenclature), followed by their function, location and/or morphology (level two nomenclature). This classification yields three main groups of cells namely, embryonic-derived macrophages, monocyte-derived cells and dendritic cells (DCs). Resident macrophages can be found in all body tissues and fulfil crucial homeostatic functions. They can be named either by location or historical discovery and depend on Csf-1R signaling for their development. Monocytes derive from BM precursors and are mainly found circulating in the blood stream. Upon inflammatory stimuli they can infiltrate body tissues and give rise to moncyte-derived cells (MC) with properties of macrophages and DCs. Bona fide DCs are dependent on Flt3 ligand for their development and can be further subdivided into 'classical' DCs (cDCs) and plasmacytoid DCs (pDCs) which arise from discrete committed precursors. Distinct DC subset are commonly distinguished by their location and surface marker profile. (adapted from ${ }^{17}$ ) 
Monocytes have long been classified merely as the definitive precursors of MFs and some DCs despite their unique functions as mononuclear phagocytes ${ }^{16}$. Nowadays they are appreciated as an individual cell lineage within the MPS based on their origin from a so-called common monocyte progenitor (cMoP) ${ }^{18}$. Two subsets of monocytes can be distinguished namely the classical monocytes, characterized by their Ly6 $\mathrm{C}^{\text {hi }}$ (mouse) or $\mathrm{CD} 14^{+} \mathrm{CD} 16^{-}$(human) marker expression, and non-classical monocytes being Ly6 $\mathrm{C}^{\text {low }}$ (mouse) or $\mathrm{CD} 14^{\text {low }} \mathrm{CD} 16^{+}$(human) ${ }^{17}$. Classical monocytes can be found in the blood as well as in several tissues during steady state. They are the definitive precursors of several mononuclear phagocytes in adult tissues including gut, heart and dermis that rely on the constant replenishment by circulating monocytes. Most likely classical monocytes also give rise to the non-classical monocytes circulating and patrolling the blood vessels, even though this is still a matter of debate ${ }^{19}$.

Under inflammatory conditions there is a dramatic increase of classical monocytes in the inflamed tissues, which in turn differentiate to monocyte-derived cells (MC) including so called 'inflammatory macrophages' or 'monocyte-derived DCs' (MoDCs) with distinct phenotypes and functions depending on the environmental context. These include similarities to DCs, including migration to lymph nodes and activation of $T$ cells, or to macrophages, including phagocytosis of pathogens and tissue repair, as well as immune-suppressive properties. Overall, monocytederived cells are often highly heterogeneous even within one inflamed organ and can outnumber the resident DCs and MFs ${ }^{17}$. 
DCs are considered the most specialized APCs as they express high levels of MHC-II on their surface to present processed antigens to T cells, prime those and initiate adaptive immune responses. In addition, DCs also promote peripheral tolerance during steady state conditions by capturing and presenting self-antigens and chronically found environmental proteins ${ }^{20}$. Developing from a common DC progenitor in the bone marrow all DCs depend on the cytokine FMS-like tyrosine kinase 3 ligand (FLT3L) while additional specific transcription factors are required to form individual subsets. The three main subsets comprise two lineages of classical or conventional DCs (cDCs), namely $\mathrm{CDC} 1$ and $\mathrm{CDC} 2$, as well as plasmacytoid DCs (pDCs) (Figure 2).

$\mathrm{cDCs}$ originate from an intermediate pre-cDC precursor that migrates from the $\mathrm{BM}$ to peripheral organs and differentiates locally into $\mathrm{CD}^{+} \mathrm{a}^{+} / \mathrm{CD} 103^{+}$(mouse) or $\mathrm{CD} 141^{+}$(human) CDC1s or CD11 $\mathrm{b}^{+} / \mathrm{CD} 172 \mathrm{a}^{+}$(mouse) or $\mathrm{CD} 1 \mathrm{c}^{+}$(human) $\mathrm{CDC} 2 \mathrm{~s}$ that then reside in the tissue ${ }^{17}$. In their immature state $\mathrm{CDCs}$ are highly phagocytic to take up antigens, process them and load them on MHC-II molecules within the lysosomal compartment. Upon maturation, cDCs lose some of their phagocytic activity and in turn increase their capacity to activate $T$ cells by externalization of the antigen MHC-II complex together with the up-regulation of co-stimulatory molecules such as CD80 and CD86 and adhesion molecules ${ }^{20}$.

pDCs generally derive from committed pre-pDCs in the BM and are relatively long lived but only have a limited capacity to populate non lymphoid organs under steady-state conditions ${ }^{21} 22$. As the name suggests pDCs morphologically resemble plasma cells and are less potent as APCs. However, in response to viral infections detected via TLRs pDCs characteristically release large amounts of type I IFN ${ }^{23}$. 
Tissue resident macrophages form the third entity of the MPS primarily being distinguished by their embryonic origin. In contrast to DCs that focus on initiating adaptive immune responses, the main function of macrophages is to ensure tissue homeostasis and integrity ${ }^{22}$. In line with this, macrophages have a high phagocytic potential and degradative function to clear damaged cells, cell debris or foreign pathogens during innate immune responses. They are also able to present antigen to $\mathrm{T}$ cells via MHC-II molecules, however their priming capacity of $\mathrm{T}$ cells is much less efficient compared to that of DCs ${ }^{22}$. In contrast to earlier concepts, it is now clear that the majority of tissue resident macrophages derive from embryonic precursors early during development. Several waves of hematopoietic precursors are produced in mammalian embryos that finally lead to the establishment of hematopoietic stem cells (HSC) in the $\mathrm{BM}^{24}$. During the first wave around embryonic day (E) 7.5 to E8.0, extra embryonic yolk sac (YS) precursors initiate the primitive hematopoiesis by giving rise to erythro-myeloid progenitors (EMPs) that seed all embryonic tissues. In the so called aorta-gonad-mesonephros (AMG) region EMPs start to differentiate into pre-HSC and mature HSC that colonize the fetal liver around E10.5 and establish the definitive hematopoiesis ${ }^{24,25}$. Even though EMPs seed all tissues, most tissue-resident macrophages, except microglia (discussed in detail below), are replaced by fetal liver monocytes during definitive hematopoiesis around E12.5 (Figure 3) ${ }^{26}$. Once established tissue macrophages self-maintain locally and independently of circulating monocytes under steady-state conditions ${ }^{27}$. In general, the development and survival of macrophages is highly dependent on the macrophage colony stimulating factor (M-Csf or Csf-1) receptor (Csf-1R). This has nicely been demonstrated in genetically-modified mice lacking Csf-1R, which show a grave reduction of macrophages throughout all body tissues resulting in severe developmental deficit including osteopetrosis and tooth eruption as well as reduced growth rate and increased postnatal mortality ${ }^{28}$. In line with these findings, it was shown that Csf-1R can signal via two ligands namely Csf-1 and IL-34, which have different contributions for the survival of individual macrophage populations ${ }^{29}$. Given the vast diversity of body tissues and their distinct functional requirements, it is not surprising that macrophages are a very heterogeneous population ${ }^{17}$. In addition to their general functions as immune sentinels each resident macrophage population acquires tailored functions 
imprinted by the tissue microenvironment to cope with tissue-specific requirements ${ }^{30,31}$. In the recent years strong efforts have been made to identify the unique genetic signatures of individual macrophage populations to gain insights into their respective function and modulatory pathways ${ }^{30-32}$.

Langerhans cells (LC) in the epidermis of the skin are primarily derived from fetal liver monocytes with a small contribution from YS macrophages. They are recruited to the skin prior to birth and maintain locally in the skin throughout life under homeostatic conditions ${ }^{22}$. Given their ontogeny and their development independently of FLT3L, they are classified here as macrophages even though they share many functional properties with CDCs. Similar to all macrophage populations, they rely on the signaling of Csf-1R for their development and maintenance. However, in contrast to many other macrophage populations, their development is independent of Csf-1 but requires IL-34-mediated Csf-1R signaling 29. LCs represent the first barrier for skin invading pathogens, they are highly phagocytic and important for the cross-presentation of ingested antigens, while their ability to effectively prime $T$ cells strongly depends on the respective pathogen. In addition they have a proposed role in allergenic contact hypersensitivity response ${ }^{33}$.

Similar to LCs, alveolar macrophages (AM) also derive from fetal liver monocytes that seed the fetal lung and fully mature during the first days after birth under the control of the granulocyte-macrophage colony stimulating factor (GM-Csf or Csf-2) and peroxisome proliferator-activated receptor gamma (PPARY). They are located in the alveolar cavities and are crucial for lung development and homeostasis by clearing excess surfactant proteins and pathogens, apoptotic cells or allergens that are bound to these. Loss or dysfunction of AMs leads to the accumulation of surfactant and the subsequent development of pulmonary alveolar proteinosis (PAP)-like diseases ${ }^{34}$.

Liver-resident Kupffer cells and splenic red pulp macrophages share some prominent features related to their surveillance and filtering of the blood stream. They are required to clear senescent or damaged red blood cells and are crucial for iron homeostasis by recovering it through heme metabolization ${ }^{35}$. This unique property is induced by the transcription factor Spi-C, which is also critical for the development of red pulp macrophages ${ }^{36}$. Kupffer cells in addition regulate plasma 
cholesterol levels by removing lipoproteins from the circulation and prevent systemic pathogen distribution by eliminating potentially harmful products such as microbial products, food antigens and xenobiotics ${ }^{37}$.

Furthermore, osteoclasts as fused multinucleated bone-resident macrophages are essential for normal long bone formation during embryogenesis and for bone resorption during the constant remodeling of this tissue ${ }^{38}$. While embryonically derived peritoneal macrophages for example contribute to the IgA production of specialized peritoneal B cells ${ }^{39}$.

Of note, with regards to tumor development as a specific disruption of regular immune function, macrophages play a dual role. On one hand, they can counteract tumor formation by capturing and killing malignantly transformed cells. On the other hand, they can promote tumor growth by their ability to induce angiogenesis and increase immunosuppression ${ }^{22}$.

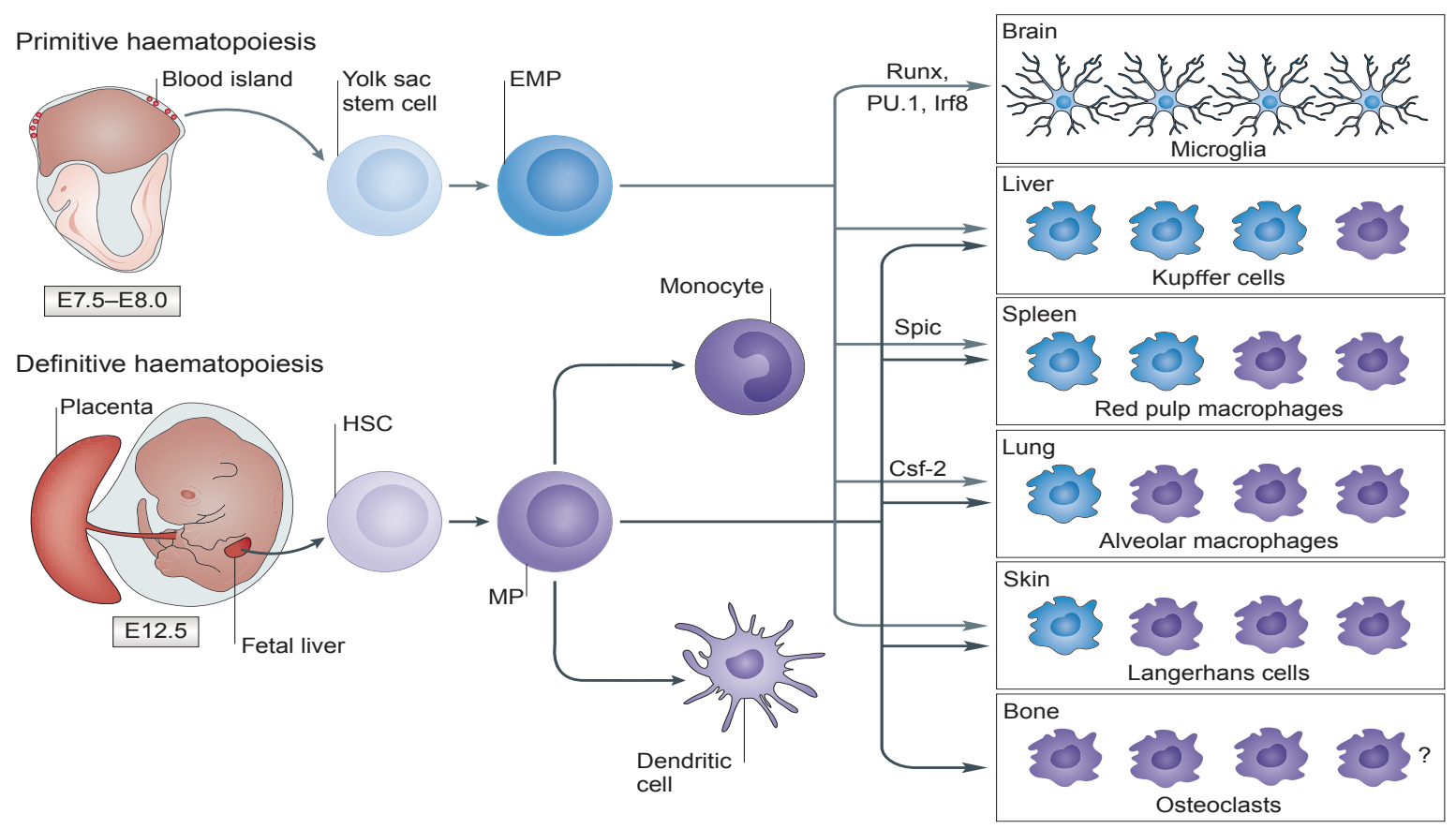

Figure 3 | Development of resident macrophages in different tissues. Several sources of myelopoiesis exist in the mouse. A transient early wave of myeloid cell development called primitive hematopoiesis takes place at embryonic day 7.5 (E7.5)-E8.0. At this time point, cells with stem cell properties develop in blood islands of the yolk sac (YS). Their progeny (erythro-myeloid progenitors (EMPs)) further differentiate into YS macrophages and populate several tissues, including the brain, where they become tissue macrophages that potentially have longevity and a high capacity for self-renewal. Shortly thereafter, myelopoiesis is taken over by progenitors found in the aorta-gonad-mesonephros region (not depicted) and fetal liver (starting at E12.5), where it forms part of the process of definitive hematopoiesis. Maturating myeloid cells derived from definitive hematopoiesis are engrafted in all tissues except the brain, where they at least partially replace the YS-derived cells. HSC, hematopoietic stem cell; MP, myeloid precursor. (adapted from ${ }^{40}$ ) 


\subsection{Immune privilege in the central nervous system}

The classical hallmarks of acute inflammation are calor (heat), rubor (redness), dolor (pain), tumor (swelling) and local loss of homeostatic tissue functions. These characteristics are the result of an active immune response including changes in vascular permeability, cell infiltration and release of immune mediating cytokines. Under normal conditions this does not affect vast body functions and tissue homeostasis is fully restored once the inflammation is resolved. For the central nervous system (CNS) even a temporary disturbance of the tissue homeostasis can have major impacts for the whole organism, which is why the CNS is relatively immune privileged. Similar exceptions are true for the eye, the placenta and the testes. In extension of earlier concepts it is now clear that this privilege is not absolute but demarcated to certain compartments and functions of the CNS ${ }^{41}$. Widely spoken it includes the CNS parenchyma while border areas such as the meninges and the choroid plexus (CP) as well as the ventricles containing the cerebrospinal fluid (CSF) are omitted. A special structural feature supporting the separation of the CNS from the rest of the body is the blood brain barrier (BBB) (Figure 4). It is established by the neurovascular unit, which contains pericytes, astrocyte end feet and cerebral endothelial cells forming extremely firm tight junctions. It also contains a specialized basement membrane formed by extracellular matrix components and harbors unique membrane transporters ${ }^{42}$. With regards to the immune response, it is mainly the afferent arm of the communication between the CNS and the immune system in the periphery that accounts for the privileged position ${ }^{41}$. Even though recent studies have identified a lymphatic system in the CNS that allows the exchange of immune cells and fluid components between the CSF and the deep cervical lymph nodes ${ }^{43}$, the communication between CNS and periphery is still tightly controlled. The emigration of APCs to the draining lymph nodes is widely reduced and these cells accumulate in the inflamed CNS. Furthermore, soluble antigens that drain along perivascular spaces and the subarachnoid space (SAS) into the cervical lymph nodes fail to elicit effective cytotoxic $T$ cell responses or delayed-type hypersensitivity. In contrast, they rather skew the immune effects towards a humoral or T helper type-2 response and even induce tolerizing effects ${ }^{41}$. In addition, the efferent arm of the immune response in the CNS is also specialized. 
Even though effector cells can reach the CSF and the CNS border areas through the cervical lymph nodes and the CNS lymphatic system ${ }^{43}$, their entry into the parenchyma is tightly regulated by the BBB. Once in the CNS, entering T cells for example face a rather pro-apoptotic and anti-inflammatory environment characterized among others by the elevated expression of the apoptosis inducing Fas ligand on CNS resident cells and the high levels of TGF- $\beta$ inducing the conversion of effector $T$ cells to Tregs ${ }^{41}$. Furthermore, also the innate immune system of the CNS is very unique being dominated by microglia as the CNSresident tissue macrophages (discussed in detail below).

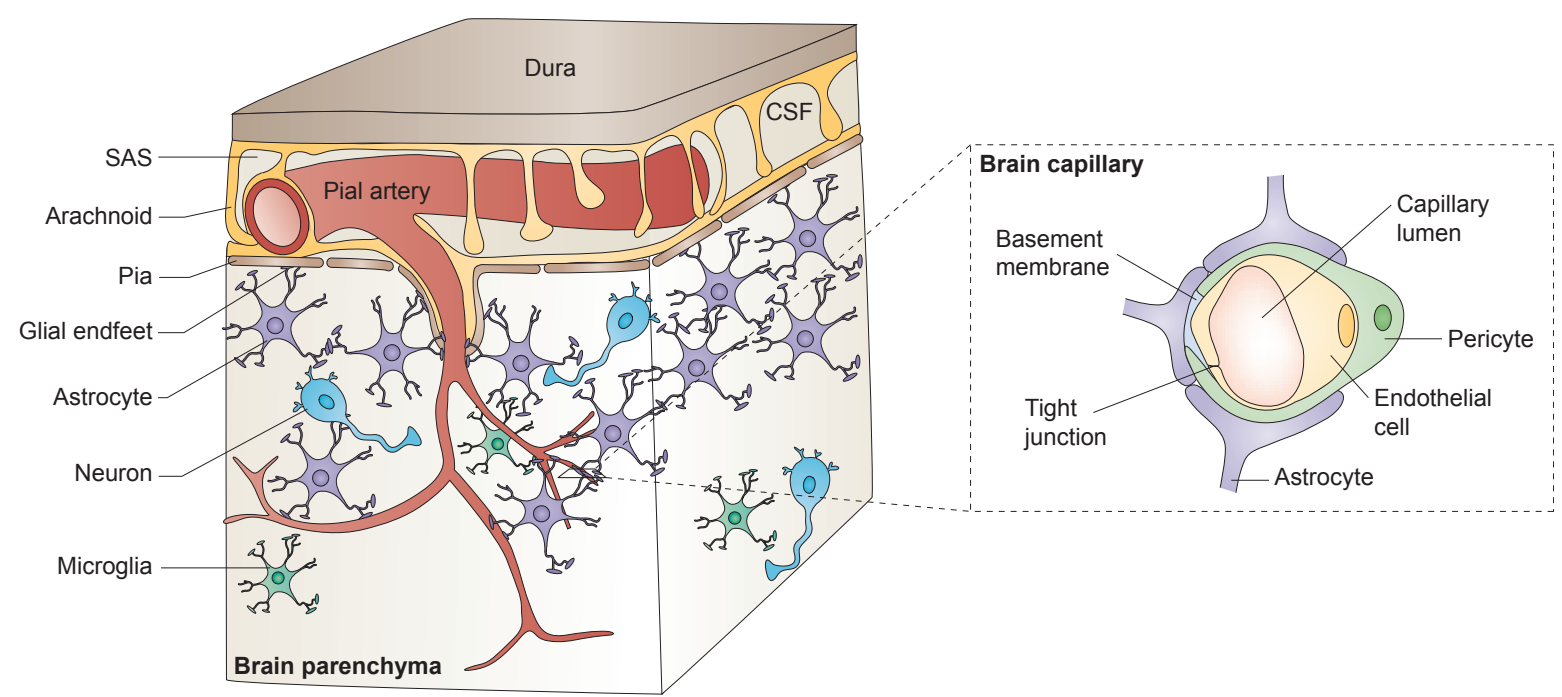

Figure 4 | Cerebral microcirculation and the neurovascular unit. In the brain, pial arteries run through the subarachnoid space (SAS), which contains the cerebrospinal fluid (CSF). These vessels give rise to intracerebral arteries, which penetrate into brain parenchyma and branch into smaller arteries and subsequently arterioles. Intracerebral arteries are separated from brain parenchyma by the pia, the innermost layer of the meninges, and the astrocyte end feet. At the brain capillary level, vascular endothelial cells and pericytes are attached to the basement membrane. Pericyte processes encase most of the capillary wall, and they communicate with endothelial cells directly through synapse-like contacts. Astrocyte end-foot processes encase the capillary wall, which is composed of endothelium and pericytes. (adapted from ${ }^{44}$ ) 


\subsection{Microglia}

\subsubsection{Origin and development}

Microglia have first been described and named by del Río-Hortega in the 1930s as a small population of phagocytic and migratory cells in the brain that are distinct from neurons and macroglia such as astrocytes and oligodendrocytes. Despite extensive research the origin and development of microglia has only been discovered in the recent years (Figure 5). The first wave of hematopoietic precursors originates from the extra-embryonic yolk sac blood islands around E7.5 and induces the primitive hematopoiesis ${ }^{24}$. During that time c-kit ${ }^{+}$erythro-myeloid precursors (EMPs) give rise to primitive yolk sac macrophages that seed the brain rudiment through developing blood vessels around E9.5 ${ }^{45,46}$. The generation these early microglia precursors is dependent on Runt Related Transcription Factor 1 (Runx1) ${ }^{47,48}$. Upon homing to the brain these primitive macrophage develop into CNS resident microglia that show a mature, ramified phenotype as early as E14 ${ }^{45}$. Similar to other macrophages, the development of microglia is strongly dependent on Csf-1R signaling which can be induced by either ligand Csf-1 or IL-34 ${ }^{46}$. Additionally, the myeloid-specific factor PU.1 (encoded by Spi1) and its interaction partner Interferon Regulatory Factor 8 (Irf8) have also been shown to be crucial for the development of microglia ${ }^{45}$. While the transcription factor $M y b$, required during definitive hematopoiesis, is dispensable for yolk sac derived macrophages ${ }^{49}$. Recent studies also propose a critical role for TGF- $\beta$ in microglia development ${ }^{50}$. The exact contribution however needs to be verified in more detail.

Under physiological conditions CNS-resident microglia are long-lived, can resist Yirradiation and maintain themselves locally without any contribution of fetal liver or BM derived monocytes ${ }^{46}$. Furthermore, different mouse models have shown that depletion of microglia leads to a rapid repopulation of the empty niche until normal microglial numbers are re-established. This repopulation is driven by CNS-intrinsic sources and microglia themselves show a high proliferative capacity ${ }^{51}$. Under certain conditions involving BBB disruption due to irradiation or injury, microglia can also be repopulated by BM-derived cells upon depletion. Even though these cells to some extend acquire a microglia phenotype and function, the genetic profile of BM-derived 'microglia-like' cells differs from that of embryonically-derived 
microglia. It remains to be shown if newly engrafted BM-derived cells can maintain the 'microglia' pool over an extended period of time and fully acquire the characteristics of embryonically-derived microglia, including steady state functions and self-maintenance ${ }^{51}$.

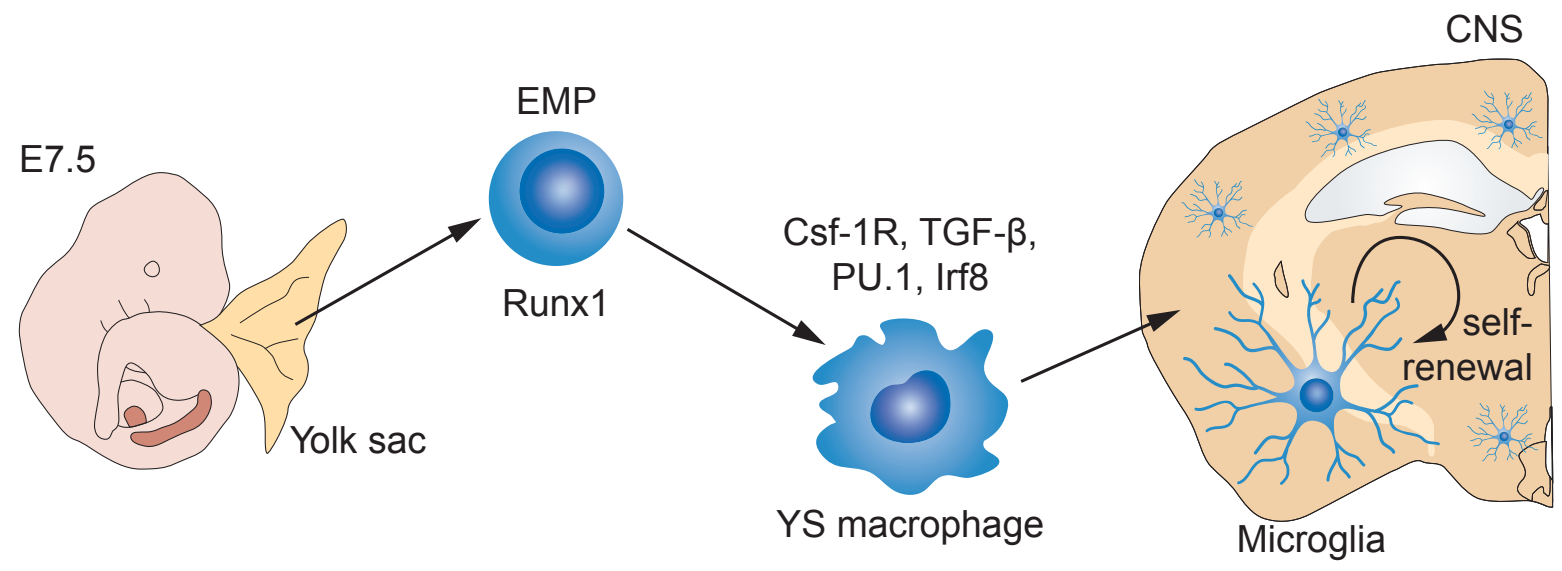

Figure 5 | Schematic for the development of microglia. During primitive hematopoiesis around E7.5 erythro-myeloid precursors (EMPs) give rise to yolk sac (YS) macrophages that seed the brain rudiment through developing blood vessels around E9.5. The generation of EMPs is dependent on Runt Related Transcription Factor 1 (Runx1) while the further development into mature microglia is strongly dependent on the myeloid-specific factor PU.1 and its interaction partner Interferon Regulatory Factor 8 (Irf8) as well as on colony stimulating factor 1 receptor (Csf-1R) and transforming growth factor beta (TGF- $\beta$ ) signaling. Under steady state conditions microglia have the capacity to self-renew and maintain locally without the contribution of circulating monocytes.

\subsubsection{Other CNS resident myeloid cells}

Apart from microglia located in the CNS parenchyma, additional CNS-associated macrophages (CNS-MF) can be found at the CNS borders namely the perivascular (pv) space, the meninges and the choroid plexus (CP). Recent studies have shown that these border areas are also seeded early during embryonic development by macrophage progenitors ${ }^{52}$, even though the specific origin of these progenitors from YS or fetal liver remains to be identified. Similar to microglia, the development of CNS-MF is highly dependent on Csf1r and PU.1 but independent of Myb. In addition, genetic deletion of Ifr8 resulted in a reduction of meningeal MFs (mMF) while the numbers of CP MF remained unchanged indicating distinct dependences 52 . Along the same lines, fate mapping studies showed that pvMF and mMF retain their embryonic origin throughout adulthood whereas CP MF were replaced over time by circulating monocytes. Despite their longevity under steady state conditions 
unlike microglia all CNS-MF are sensitive to $\mathrm{y}$-irradiation and also display a more amoeboid morphology ${ }^{52}$. The different contributions of microglia and CNS-MF to CNS homeostasis and neuroinflammation will be discussed in the following chapters.

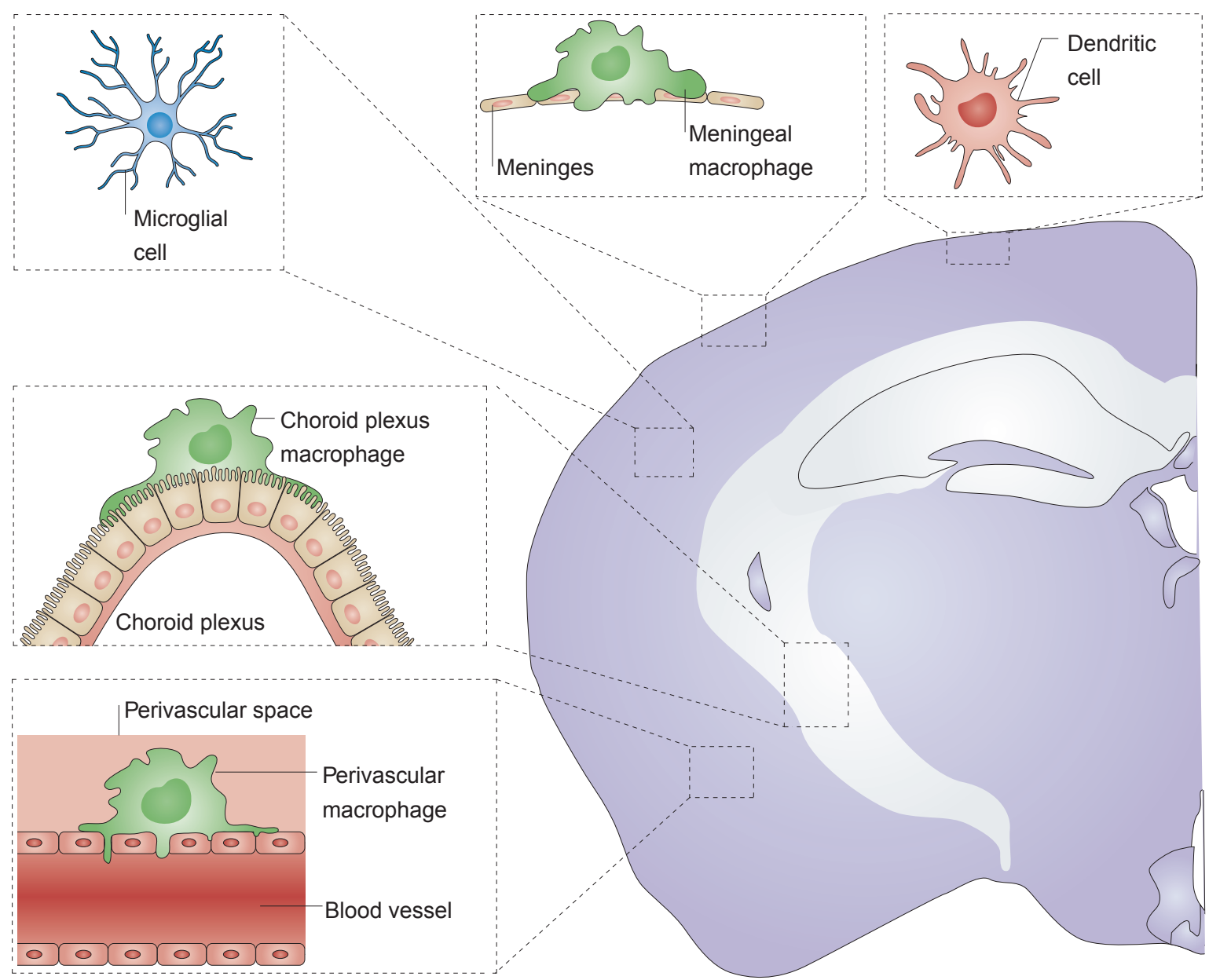

Figure 6 | Myeloid cell types in the CNS. Under homeostatic conditions, the brain hosts several heterogeneous populations of myeloid cells that are located at distinct sites, where they execute homeostatic and surveillance tasks. Within the brain parenchyma, microglia with small delineated processes actively screen the intraneuronal space for incoming threats, whereas macrophages can be found in the outer boundaries of the brain, such as the choroid plexus, perivascular space, and in the meninges. DCs are present at low numbers in the same locations as macrophages. (adapted from ${ }^{40}$ ) 


\subsubsection{Homeostatic functions}

Despite intensive investigations it remains difficult to properly define the homeostatic functions of microglia within the CNS. Doubtlessly they are the local innate immune cells and represent the first line of defense against invading pathogens and damaging insults. Microglia constantly scan the CNS parenchyma with their highly motile processes and clear cellular debris and accumulated products to restore tissue homeostasis ${ }^{53}$. In addition to their immune functions, it has been shown that microglia play a key role during brain development and proper formation of neuronal circuits by a process called synaptic pruning and the engulfment of synaptic material as well as apoptotic neurons ${ }^{54}$. Even more so, in the cerebellum microglia can actively induce apoptosis of developing Purkinje neurons by so called respiratory burst that is characterized by the rapid release of reactive oxygen species (ROS) ${ }^{55}$. A transient reduction of microglia numbers and/or decreased recognition of synapses before and during engulfment e.g. by the depletion of the fractalkine receptor $\left(\mathrm{CX}_{3} \mathrm{CR} 1\right)$ results in an excess of dendritic spines and an increase in synaptic release sites indicative of immature synapses 54. Along those lines, mice lacking $\mathrm{CX}_{3} \mathrm{CR} 1$ show abnormalities in social interactions and repetitive behaviors as a result of neurodevelopmental changes ${ }^{56}$. The cytokine receptor $\mathrm{CX}_{3} \mathrm{CR} 1$ on the cell surface of microglia represents one of the main interaction pathways between microglia and neurons. Under physiological conditions $C X_{3} C R 1$ responds to its ligand fractalkine $\left(C X_{3} C L 1\right)$ mainly produced by neurons to restrain microglia activity and maintain their surveying phenotype ${ }^{57}$. Similar crosstalk to inhibit excessive microglia activation is established through the CD200R - CD200, CD172 - CD47 and CD45 - CD22 axes, in which microglia always carry the receptors for the respective neuronal ligands ${ }^{58}$. In addition to cellular interactions, soluble factors secreted by microglia critically influence neuronal development and function. Among those brain-derived neurotrophic factor (BDNF) is one of the best studied so far and has been shown to modulate neuronal plasticity, synaptic remodeling and neurotransmission especially in glutamatergic neurons assigning an important role during learning and memory formation to microglia ${ }^{59}$. Interestingly the crosstalk between neurons and microglia is not onedirectional but neurons also tune microglia activities by means of their own activity levels, the response to visual stimuli and the strength of their synapses ${ }^{60,61}$. 
Furthermore, recent evidence suggests that microglia are crucially involved in adult neurogenesis in the subgranular zone (SGZ) of the hippocampus by phagocytosis of excessively generated, apoptotic neurons without promoting inflammation ${ }^{62}$. In fact, it has been shown that pro-inflammatory stimuli have a negative impact on neurogenesis ${ }^{63}$.

\subsubsection{Role during neuroinflammation}

Upon inflammatory stimuli microglia change their gene expression profile and phenotypic appearance, morphologically indicated by switch from a ramified cell body with long, dynamic processes and a small cell soma to a rather amoeboid phenotype. With regards to neurodegenerative diseases such as Alzheimer's disease (AD) and multiple sclerosis (MS) as well as aging, activation of microglia may lead to both protective but also pathogenic effects. Protective functions of microglia include phagocytosis of cellular debris, secretion of growth factors and initiation of regenerative processes to restore tissue homeostasis ${ }^{64}$. In contrast, promotion of pathogenic microglia functions result in the production of neurotoxic factors such as ROS or NO as well as the release of pro-inflammatory cytokines and chemokines including IL-1 $\beta$, tumor necrosis factor alpha (TNF $\alpha$ ) and CCL2 (Figure 7). These immune mediators may lead to the activation of astrocytes resulting in astrogliosis and promote the infiltration of BM-derived monocytes into the CNS parenchyma that differentiate into effector cells, such as monocytederived MF or DCs ${ }^{64}$. Additionally, several mutations in genes related to microglia function including DNAX-activating protein of $12 \mathrm{kDa}$ (DAP12) mediating synaptic signaling via BDNF and the PRRs triggering receptor expressed on myeloid cells 2 (TREM2) promoting phagocytosis or Nod-like receptor protein 3 (NLRP3) forming the inflammasome have been proposed to correlate to the pathogenesis of neurodegenerative diseases ${ }^{65}$.

CNS demyelination such as in MS and the related mouse model experimental autoimmune encephalomyelitis (EAE), is mainly initiated by the infiltration of autoreactive $\mathrm{CD} 4^{+} \mathrm{T}$ cells ${ }^{66}$. However, the effector mechanisms leading to disease progression are provided by CNS-resident microglia and infiltrating monocytederived cells. These myeloid cells promote the encephalitogenic potential of infiltrating $T$ cells by the secretion of pro-inflammatory cytokines such as IL-12 and 
IL-23 ${ }^{67,68}$. Furthermore, they produce chemokines, attracting additional effector cells from the periphery, and ROS, increasing the neuronal damage ${ }^{69}$. Recent studies claim that it is mainly the infiltrating monocyte-derived MF that are highly activated and have a strong disease promoting effect by initiating demyelination preferably at the nodes of Ranvier ${ }^{70}$. Whereas the resident microglia show a rather repressed gene expression profile, appear relatively inert and merely clear debris of damaged neurons ${ }^{70}$. More studies will be needed to clearly decipher the differential roles of resident microglia vs. CNS-associated myeloid cells or infiltrating myeloid-derived cells. A similar pattern seems to be true for models of amyotrophic lateral sclerosis (ALS) where the progressive recruitment of monocytes to the spinal cord promotes neuronal loss. Interestingly this recruitment seems to be mediated by microglia producing the chemoattractant CCL2 while they themselves succumb to apoptosis with advanced disease progression ${ }^{71}$.

One of the characteristics of $A D$ is the deposition and aggregation of amyloid $\beta$ $(A \beta)$. It has been shown that $L y 6 C^{\text {low }}$ monocytes patrolling the blood vessels and pvMF located at the borders are able to clear $A \beta$ from these sites and reduce the plaque burden in the $C N S^{72,73}$. The role of microglia in the clearance of $A \beta$ plaques is more controversial. Even though microglia are initially able to engulf deposited $A \beta$, increasing plaque burden in the CNS seems to inhibit their phagocytic capacity and the motility of their processes towards the site of deposition. Along the same lines, transient depletion of microglia in murine $A D$ models did not rescue plaque burden or neuritic dystrophy ${ }^{74}$. Moreover, in vitro studies suggest that microglia might contribute to $A \beta$ induced neurotoxicity by releasing ROS and NO (Figure 7) 75,76 


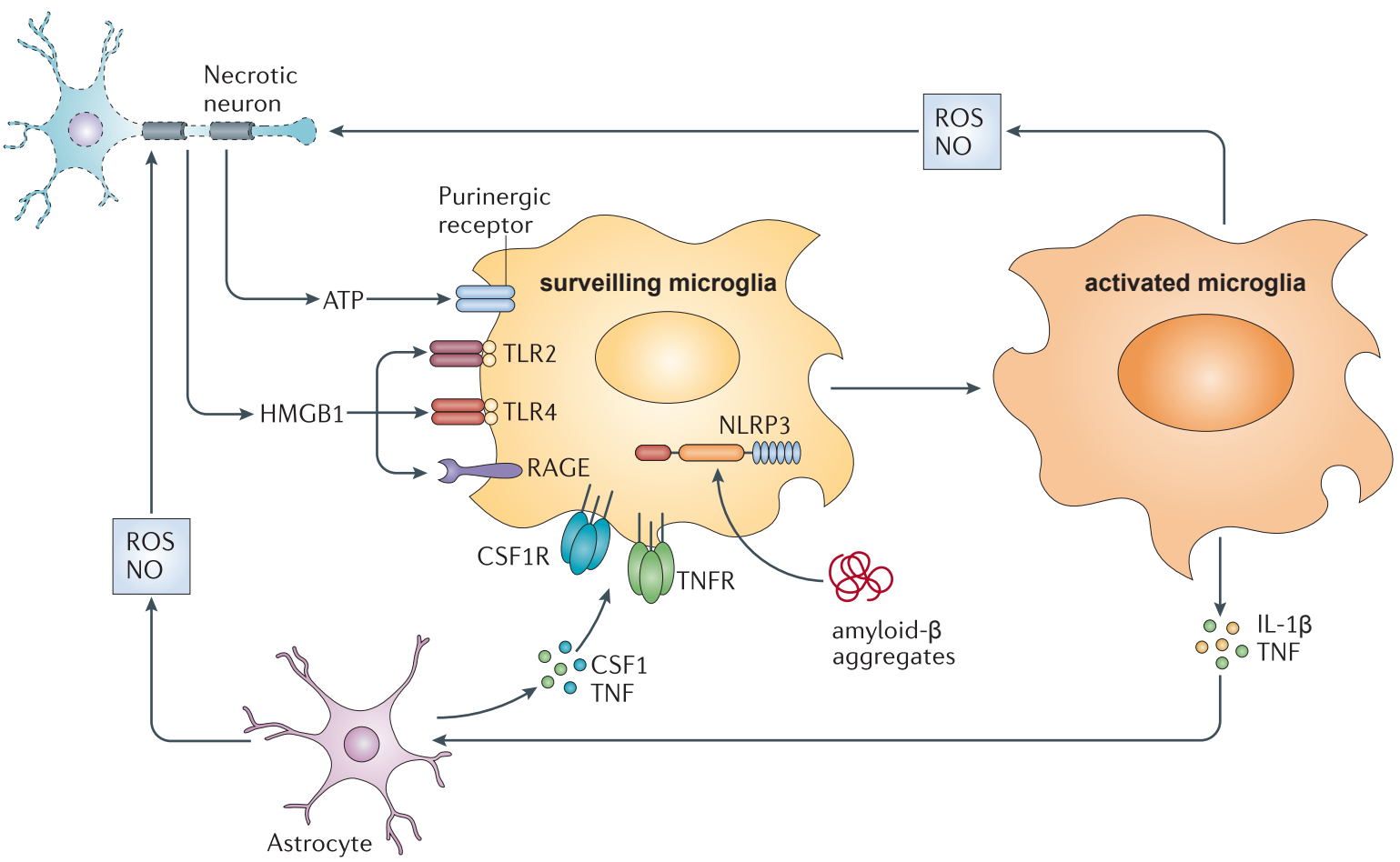

Figure 7 | Activated microglia in neurodegenerative disease. Various disease-associated factors can activate microglia through pattern-recognition and purinergic receptors to establish an activated phenotype. Such factors include, damage-associated molecular patterns (DAMPs; such as high-mobility group box 1 protein (HMGB1), histones and ATP), as well as neurodegenerative disease-specific protein aggregates (such amyloid- $\beta$ ). Pro-inflammatory mediators produced by activated microglia activate astrocytes, and the products released by activated microglia and astrocytes may exert neurotoxic effects. Activated astrocytes also release cytokines, including colony-stimulating factor 1 (Csf-1) and tumor necrosis factor (TNF), that further induce the activation and proliferation of microglia. Communication between microglia and astrocytes may therefore amplify pro-inflammatory signals initially sensed by microglia and contribute to the pathology of neurodegenerative disease. (adapted from ${ }^{64}$ )

Also for acute CNS injury it has been shown that monocyte-derived MF infiltrating the CNS promote functional recovery ${ }^{77}$. Contradicting studies however claim detrimental roles for infiltrating monocytes during spinal cord injury. One explanation for this discrepancy could be that the role of infiltrating cells depends on the time point and/or port of CNS entry, which seems to promote either a pro- or anti-inflammatory phenotype ${ }^{77}$. Similarly, microglia that accumulate in the core of the lesion and the surrounding penumbra play dual roles by contributing to the demise of neurons and exacerbation of the lesion size through synthesis of proinflammatory mediators on the one hand. And on the other hand forming the prerequisite for synaptic regeneration by scavenging cellular debris ${ }^{78}$. 
In addition to disease settings, aging has an important influence on microglia function and CNS homeostasis as it is characterized by increased oxidative stress and low-grade inflammation ${ }^{79}$. Aged microglia display a more activated phenotype indicated by morphological changes and constitutive secretion of TNFa and IL-6. Furthermore, a decline in lysosomal and mitochondrial functions in microglia during aging results in the excessive generation of ROS and an increased cytokine response to insults ${ }^{80}$.

Taken together, these examples show that microglia, CNS-associated MF and monocyte-derived cells play distinct, non-redundant roles in settings of CNS injury and degeneration. These functions are highly regulated and can be both detrimental and protective depending on the specific inputs. Further studies will be needed to fully understand the underlying mechanisms regulating myeloid cell functions in the CNS and to allow for beneficial manipulation.

\subsection{Transcription factors and genetic regulation}

Genetic regulation is the most fundamental ways of inducing, controlling, regulating and coordinating all processes in the body. Even though it follows similar concepts, genetic regulation varies between species, individuals and even single cells. Exemplarily, this becomes clear with regards to the previously mentioned differences between individual tissue resident macrophage populations, where the specific identity and function is imprinted by a unique genetic signature ${ }^{30-32}$. In contrast to functional specialization, alterations in the genetic signature e.g. by mutations in regulatory sequences or transcriptions factors can also be the cause of many different diseases including cancer, autoimmunity and neurological disorders. 


\subsection{Concepts of gene regulation}

The deoxyribonucleic acid (DNA) consists of a huge variety of genes that are transcribed into protein-coding and noncoding ribonucleic acid (RNA). The proteincoding RNA, encoded on exon sequences, will be translated into amino acid (AA) sequences by ribosomes, which undergo further post-translational modifications before they finally get folded into functional proteins. Each step of this protein biosynthesis pathway is tightly regulated and the main concepts of this regulation on a genetic level will be described as follows.

Transcriptional regulation generally occurs on two interconnected levels, namely transcription factors and the transcriptional apparatus itself, as wells as on the chromatin level including the DNA-packaging histones (Figure 8) ${ }^{81}$.

Transcription factors (TF) form a complex network of genetic regulations and can be divided into initiation or elongation controlling elements with most TF contributing to transcription initiation. They typically bind to enhancer elements in the promoter regions of genes together with other TF and recruited cofactors in a cooperative fashion. The cofactors have modulating functions themselves and can be either activating or repressive but they are unable to bind DNA directly. For each TF complex located to the core promoter region of a gene, all inputs of the individual participants will be combined and define the regulatory outcome on the initiation of the DNA transcription by the enzyme RNA polymerase II (Pol II). After transcribing a short distance Pol II is stopped by pause control factors and additional TF are required to release Pol II from these pause sites ${ }^{81}$.

The second level of modulation is related to the basic chromatin unit, the nucleosome that consists of a DNA segment wrapped around a core of eight histone proteins. Chromatin remodeling processes, partially induced by TF, lead to the mobilization and accessibility of nucleosomes and the recruitment of histone modifying enzymes resulting in an open euchromatin vs. condensed heterochromatin structure. Common histone modifications are acetylation, methylation and ubiqutinylation, which are induced and removed in a highly dynamic fashion. The activation state of a specific gene can be identified by its characteristic chromatin modifications, which allows the differentiation not only 
between active and repressed genes but also between genes that are temporary repressed but poised for later activation and those that are fully silenced ${ }^{81}$.

In addition to the two major levels of transcriptional modulation there is also noncoding RNA (ncRNA) that is usually transcribed from intron sequences of genes. ncRNA contributes to the control of gene expression through the modulation of transcriptional and post-transcriptional processes. There are different classes of ncRNA, the best studied one being microRNAs, which are only about 22 nucleotides of length and modulate gene expression at the level of messenger RNA (mRNA). Additionally, there are long ncRNAs with contribute to transcriptional modulation by recruitment of chromatin regulators ${ }^{81}$. Furthermore, so-called enhancers are genetic elements that increase the transcription of genes independent of their orientation and distance relative to the promoter region. They can be located within intron sequences of the same gene, several kilo base pairs (kb) upstream or downstream of the promoter and due to chromosome pairing even on a different chromosome ${ }^{82}$.

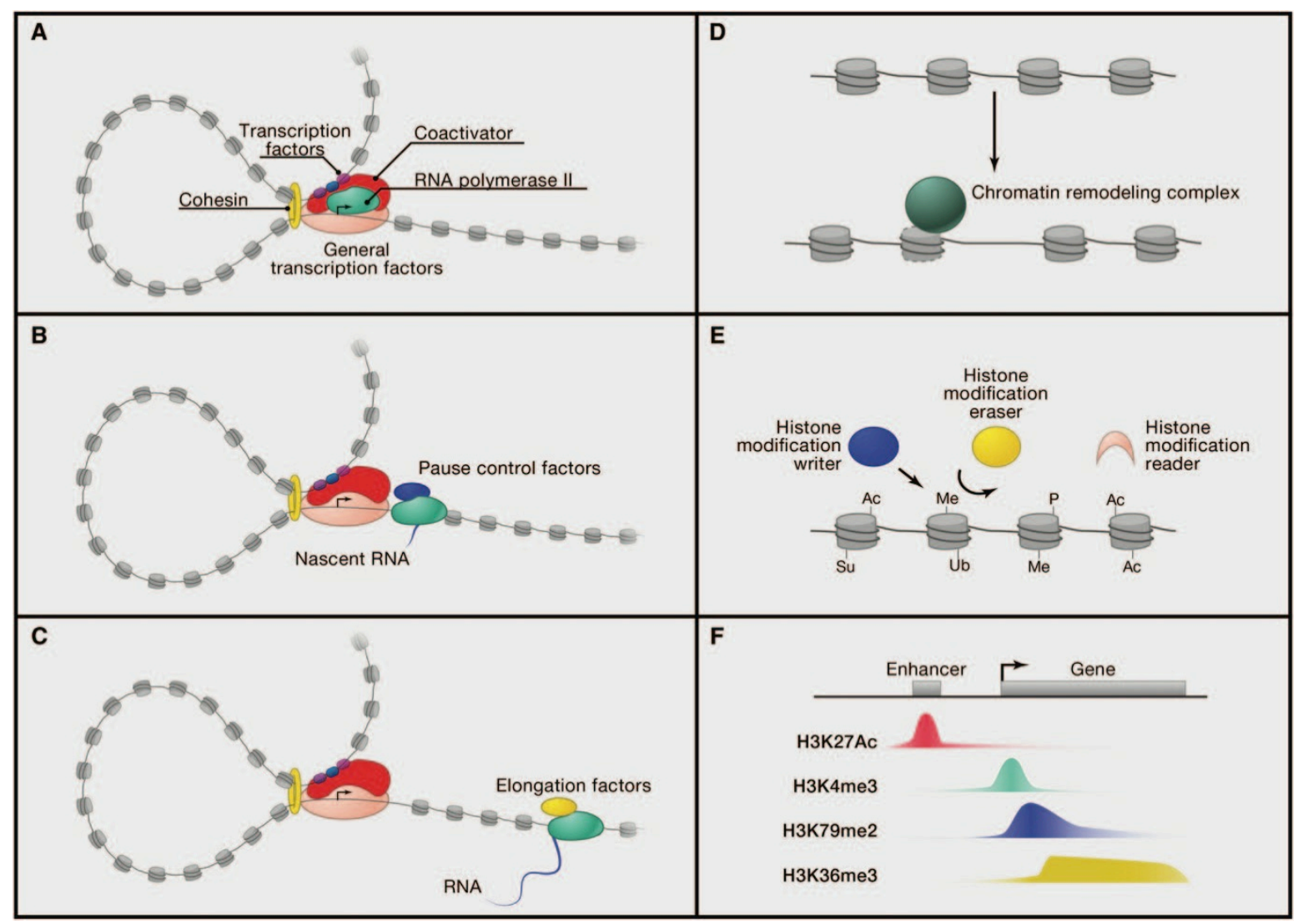

Figure 8 | Transcriptional regulation 
(A) Formation of a pre-initiation complex. Transcription factors bind to specific DNA elements (enhancers) and to co-activators, which bind to RNA polymerase II, which in turn binds to general transcription factors at the transcription start site (arrow). The DNA loop formed between the enhancer and the start site is stabilized by cofactors such as the Mediator complex and cohesin. (B) Initiation and pausing by RNA polymerase II. RNA polymerase II begins transcription from the initiation site, but pause control factors cause it to stall some tens of base pairs downstream. (C) Pause release and elongation. Various transcription factors and cofactors recruit elongation factors, which phosphorylates the pause release factors and polymerase, allowing elongation to proceed. (D) Chromatin structure is regulated by adenosine triphosphate (ATP)-dependent remodeling complexes that can mobilize the nucleosome, allowing regulators and the transcription apparatus increased access to DNA sequences. (E) Transcriptional activity is influenced by proteins that modify and bind the histone components of nucleosomes. Some proteins add modifications (writers), some remove modifications (erasers), and others bind via these modifications (readers). The modifications include acetylation (Ac), methylation $(\mathrm{Me})$, phosphorylation $(\mathrm{P})$, sumoylation $(\mathrm{Su})$, and ubiquitination $(\mathrm{Ub})$. (F) Histone modifications occur in characteristic patterns associated with different transcriptional activities. As an example, the characteristic patterns observed at actively transcribed genes are shown for histone $\mathrm{H} 3$ lysine 27 acetylation (H3K27Ac), histone H3 lysine 4 trimethylation (H3K4me3), histone H3 lysine 79 dimethylation (H3K79me2), and histone $\mathrm{H} 3$ lysine 36 trimethylation (H3K36me3). ${ }^{81}$

\subsection{Zinc finger transcription factor Sall1}

As mentioned before, tissue-specific identity of macrophages is tightly regulated by unique signature genes throughout development and adulthood. Regarding microglia, several signature genes have recently been proposed, among those Sall1 ${ }^{32,50,70,83,84}$.

Sall1 is one of the four murine members of the spalt (spalt like, Sall) genes, that have originally been identified in Drosophila and are evolutionarily highly conserved from $\mathrm{C}$. elegans to vertebrates ${ }^{85}$. Spalt genes are a family of zinc finger transcription factors carrying several conserved zinc finger domains mostly clustered in duplex or triplets as well as a glutamine-rich (polyQ) region (Figure 9). The exact functions of individual domains remain to be established, some are probably involved in DNA binding while others account for protein-protein interactions and transactivation ${ }^{86}$. Sall1 for example has been shown to mediate transcriptional repression by the recruitment of the nucleosome remodeling and deacetylase (NuRD) complex ${ }^{87}$. In contrast to that Sall1 has also been shown to synergistically activate canonical, $\beta$-catenin-dependent Wnt-signaling via binding to heterochromatin within the cell nucleus and altering its structure ${ }^{88}$. This could imply a role of Sall1 in Wnt-related processes such as embryogenesis but also cancer development. In addition to transcriptional regulations Sall1 might also be involved in post-translational modifications by modulating sumoylation of proteins. Sumoylation, in contrast to the closely related process of ubiquitinylation, does not 
mark proteins for degradation but rather alters protein affinity, localization or function and possibly even protects from ubiquitin-mediated proteolysis ${ }^{89}$.

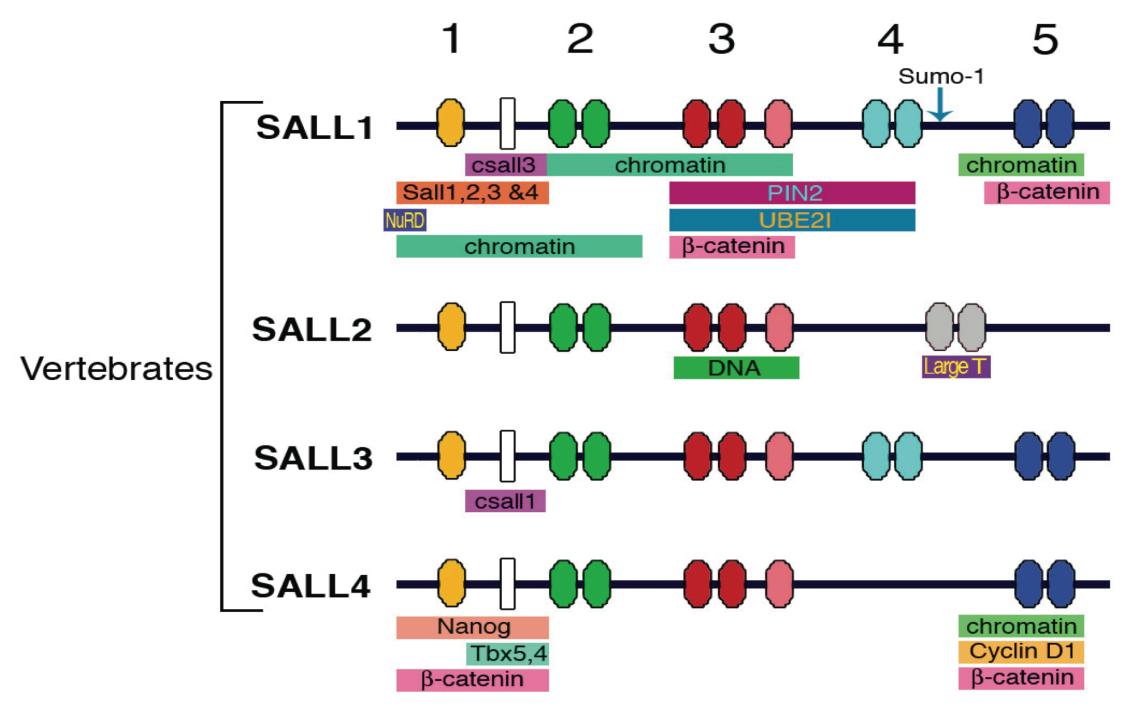

Figure 9 | Schematic representation of the main conserved domains present in Sall proteins. Colored ovals numbered 1 to 5 represent the zinc finger domains from vertebrate Sall homologues. White rectangles represent the poly-glutamine regions. The arrow in Sall1 indicates the sumoylation site described for this protein. Colored horizontal bars below each protein indicate the Sall-interaction domains with other proteins. Vertebrate data were collected from human, mouse, chicken and frog homologues. (adapted from ${ }^{90}$ )

As already indicated, spalt genes have been shown to play a crucial role during embryogenesis where they are required for the normal development of different organ systems including limbs, kidney, CNS and heart. Even though very little is known about the exact regulations and interactions of spalt genes, a common concept is the involvement in patterning and co-ordination during organogenesis. This has been nicely demonstrated for the kidney where Sall1 is essential for uteric bud invasion as the initial step of metanephros development ${ }^{86}$. In the developing embryo Sall1 is expressed in the metanephric mesenchyme surrounding the uteric bud and induces its outgrowth into the adjacent tissue. Reciprocal interaction of both tissues and branching of the uteric bud induces tubule formation and forms the basis for normal kidney development. In Sall1 knockout mice the key inducing signal is missing, which eventually leads to the apoptosis of the mesenchyme and the mice die within the first $24 \mathrm{~h}$ after birth due to severe kidney dysgenesis or agenesis ${ }^{86}$. Similar to the kidney, Sall1 also seems to take over crucial inducer functions during limb and heart development ${ }^{85}$. Spalt genes are also expressed 
during CNS development across species. In mice Sall1 shows a robust expression in early progenitor cells and promotes terminal neurogenesis ${ }^{91}$. In the cerebral cortex this is achieved first by the generation of radial glial cells from symmetric division of progenitor cells and later by the asymmetric division of these radial glial cells into intermediate progenitors that ultimately give rise to mature neurons. Sall1 seems to regulate all these steps preferentially promoting proliferation over differentiation ${ }^{91}$. Further investigations are needed to elucidate the spatio-temporal regulation of Sall1 and other spalt genes to better understand their role in tissue development and patterning.

\subsection{SALL1-deficiencies in human}

In humans, heterozygous mutations of SALL1 lead to Townes-Brocks syndrome (TBS), an autosomal dominant developmental disorder. Characteristic features of TBS are anomalies of hand and feet, anorectal abnormalities, deformities of the outer ear, along with hearing impairments and in several cases also malformations of the kidney. Less common symptoms can also be heart anomalies and mental retardation ${ }^{92}$. These symptoms, especially the kidney phenotype, correlate to some extend to what has been observed in mice ${ }^{86}$. The mutations of the SALL1 gene leading to TBS often cause a premature stop codon in the gene sequence terminating the transcription before the functionally relevant zinc finger domains. Furthermore, the truncated SALL1 protein interferes in a dominant-negative manner with the function of its full-length counterpart, rendering both proteins unfunctional ${ }^{85}$. In contrast to TBS, homozygous, autosomal recessive mutations in SALL1 can lead to 'multiple congenital anomaly-mental retardation syndrome', characterized by prenatal onset of chronic renal failure and severe CNS involvement ${ }^{93}$. As indicated earlier, SALL1 could also play a role in tumorigenesis by its ability to enhance Wnt signaling and has been reported at high levels in Wilms tumor ${ }^{85}$. Firm evidence for these implications is still missing and given the diverse functions of SALL1 it could possibly also act as a tumor suppressor. 


\section{Specific Aims}

Microglia are the resident macrophages of the central nervous system. In addition to their function as innate immune cells in this immune privileged organ, microglia also play a crucial role in tissue homeostasis and during inflammation. Despite intensive research over the past decades, their exact contributions remain to be defined. One of the reasons that our understanding of microglia biology is still relatively limited is the fact that conditional gene-targeting of microglia often leads to inadvertent targeting of other members of the mononuclear phagocyte system, including monocytes and macrophages. Recent efforts have been made to identify unique signature genes of individual resident tissue macrophage populations. In line with this the transcription factor Sall1 has been proposed to be specifically high expressed in microglia compared to other MPS members. Within the scope of this thesis the following aims will be addressed:

We want to verify that Sall1 is specifically expressed by microglia but not by any other member of the MPS or hematopoietic system.

Utilizing the tamoxifen-inducible Sall $1^{\mathrm{CreER}}$ mouse strain, we aim to modulate and target microglia in vivo.

Establishing this novel targeting scheme, our goal is to exploit Sall1 to better understand microglia biology and function under different conditions.

Given the high expression levels of Sall1 in microglia, we aim to determine the role of this transcription factor in microglia during development, steady state and inflammation. 
Specific Aims 


\section{Results}

\subsection{Microglia-specific expression of Sall1}

Microglia are the resident tissue macrophages of the CNS and pose a specialized sub-population of the MPS ${ }^{94}$. Given their highly specialized functions depending on the tissue environment they reside in, each macrophage subset has a unique gene expression signature. In the case of microglia this signature includes several transcription factors, among them Sall1 ${ }^{32}$. In order to validate that Sall1 was specifically expressed by microglia but not by other members of the hematopoietic system, we used Sall $1{ }^{\mathrm{GFP} / \mathrm{+}}$ reporter mice ${ }^{95}$. Using flow cytometry, we analyzed the CNS of Sall ${ }^{\mathrm{GFP} /+}$ mice where we detected a population of $\mathrm{GFP}^{+}$cells among the $\mathrm{CD} 45^{+}$cells, which corresponded to microglia, characterized as $\mathrm{CD} 45^{10} \mathrm{CD} 11 \mathrm{~b}^{+}$ cells. Furthermore, by gating first on microglia we could show that all of these cells express GFP (Figure 10a). To investigate whether other hematopoietic cells also express Sall1 we sorted different leukocyte populations from peritoneum, spleen, lung and BM and compared their Sall1 expression levels to that of microglia using qPCR. We did not detect Sall1 expression in any other hematopoietic population apart from CNS resident microglia (Figure 10b). Additionally, we analyzed different lymphoid and non-lymphoid organs of Sall $1^{\mathrm{GFP} /+}$ mice by flow cytometry and failed to detect GFP expression in any hematopoietic $\left(\mathrm{CD} 45^{+}\right)$cells outside of the CNS (Figure 10c). 


\section{Results}

a

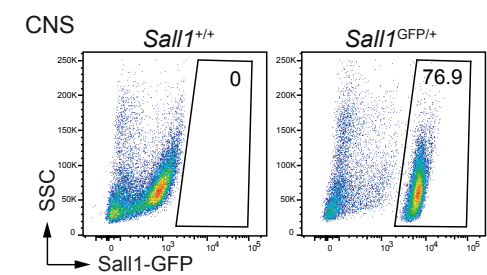

c

Blood

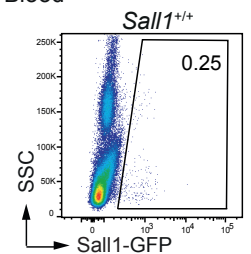

Lymph node

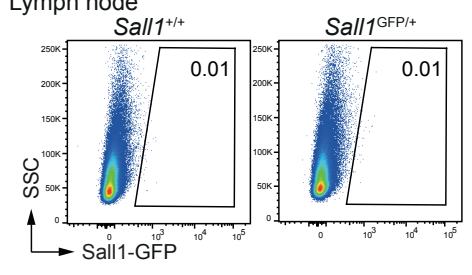

Liver

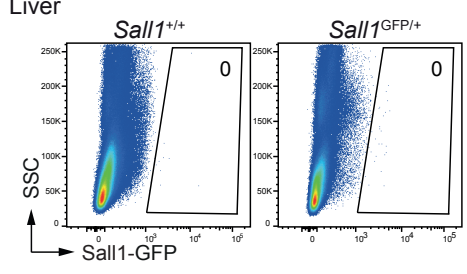

Skin

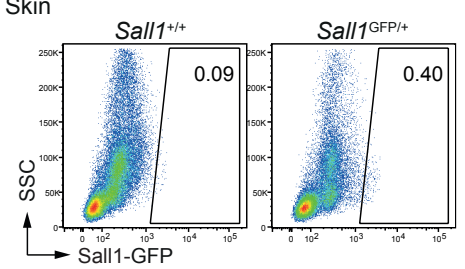

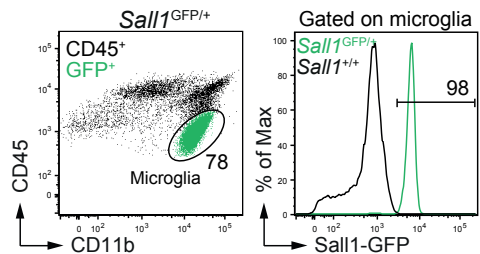

BM

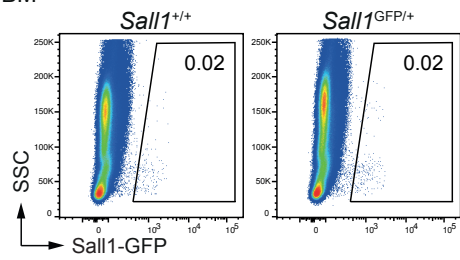

Spleen Sall1 ${ }^{+/+}$

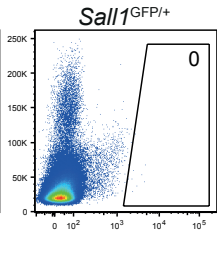

Lung
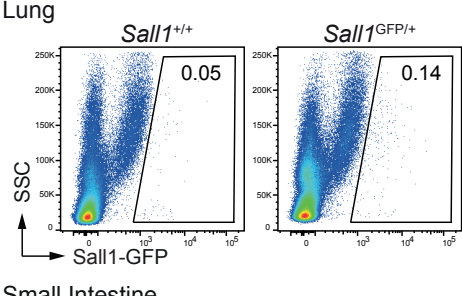

Small Intestine
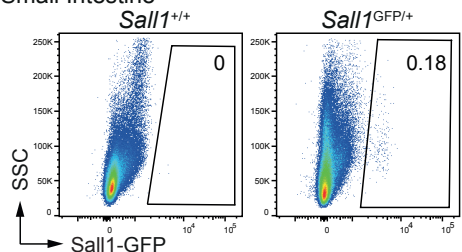

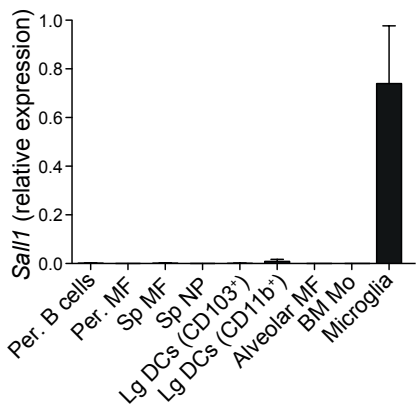

Thymus

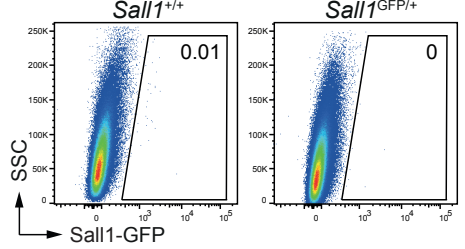

Kidney
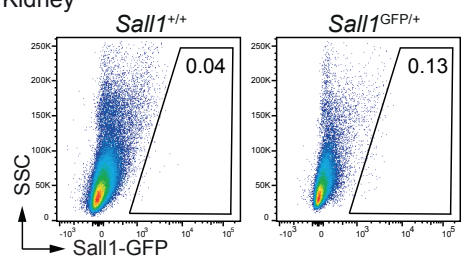

Heart
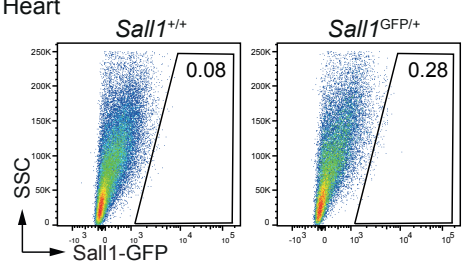

Testis
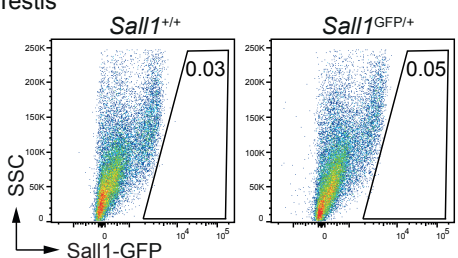

Figure 10 | Sall1 expression is restricted to the CNS within the hematopoietic system. (a) Flow cytometry analysis of GFP (Sall1) expression in CNS of Sall $1^{\mathrm{GFP} /+}$ and Sall1 ${ }^{+/+}$control mice. Cells were pre-gated on CD $45^{+}$cells. Shown are representative flow cytometry plots of at least 5 independent experiments $(n>5)$. (b) Quantitative RT-PCR of Sall1 mRNA expression ( \pm SEM) of sorted cell populations derived from WT mice.

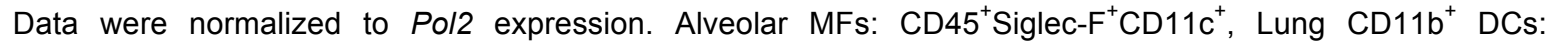

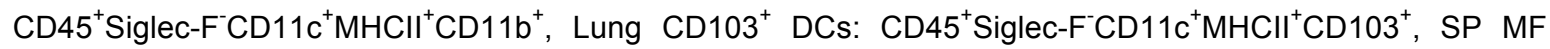
(spleen macrophages): $\mathrm{F} 4 / 80^{\mathrm{hi}} \mathrm{CD} 11 \mathrm{~b}^{+}$, SP NPs (neutrophils): Ly6G ${ }^{+} S S C^{\text {hi }}$, BM Monocytes (Mo): LinCD11 $\mathrm{b}^{+}$Ly6C $\mathrm{C}^{+} \mathrm{CD} 115^{+}$, microglia: CD45 ${ }^{10}$ Ly6C $\mathrm{Cy}^{-} \mathrm{GG}^{-} \mathrm{CD} 11 \mathrm{~b}^{+} \mathrm{F} 4 / 80^{+}$, Per. B cells (peritoneal B cells): B220 ${ }^{+}$, peritoneal MFs: $C D 115^{+} \mathrm{CD} 11 \mathrm{~b}^{+} \mathrm{F} 4 / 80^{+}$. $(n=2-3)$. (c) Flow cytometry analysis of GFP (Sall1) expression in different organs of Sall $1^{\mathrm{GFP} /+}$ and Sall $1^{+/+}$control mice. Cells were pre-gated on $\mathrm{CD} 45^{+}$cells (shown are representative flow cytometry plots, $n \geq 2$ ). 
It has been described previously that Sall1 is expressed in kidney cells where it plays an essential role during organogenesis ${ }^{86}$. Indeed, when we analyzed different whole tissue lysates of lymphoid and non-lymphoid organs from WT mice by qPCR, we found detectable levels of Sall1 not only in the brain and spinal cord, but also in kidney, liver and heart (Figure 11a). Similar results where obtained by flow cytometry in Sall1 ${ }^{\mathrm{GFP} /+}$ mice, where we detected Sall1 expression among nonhematopoietic cells (CD45) in the liver, kidney and to a small extent in heart tissue (Figure 11b,c). In the liver a small number of mainly epithelial cells (Epcam ${ }^{+}$) were found to be GFP ${ }^{+}\left(14 \%\right.$ of $\mathrm{CD}^{-} 5^{-}$cells) and in the heart, the negligible number of GFP $^{+}$cells $(1 \%)$ correlated to endothelial cells $\left(C D 31^{+}\right)$(Figure 11b). We found $12 \% \mathrm{GFP}^{+}$cells (among CD45 cells) in the adult kidney where Sall 1 is expressed in the renal tubular epithelia and in stem cells as previously described ${ }^{96,97}$.

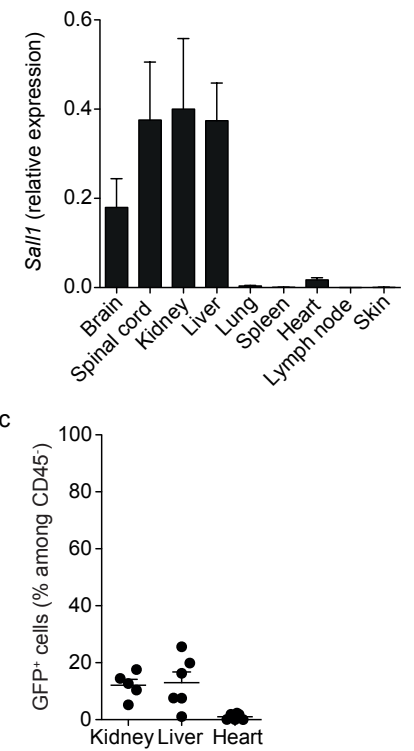

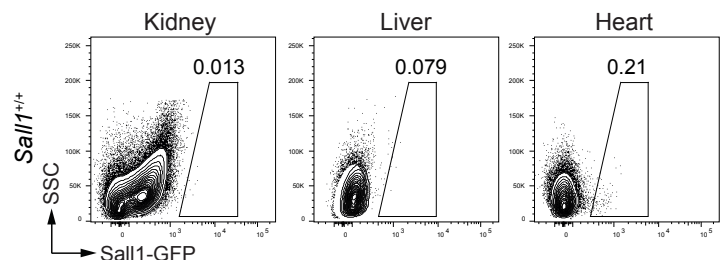
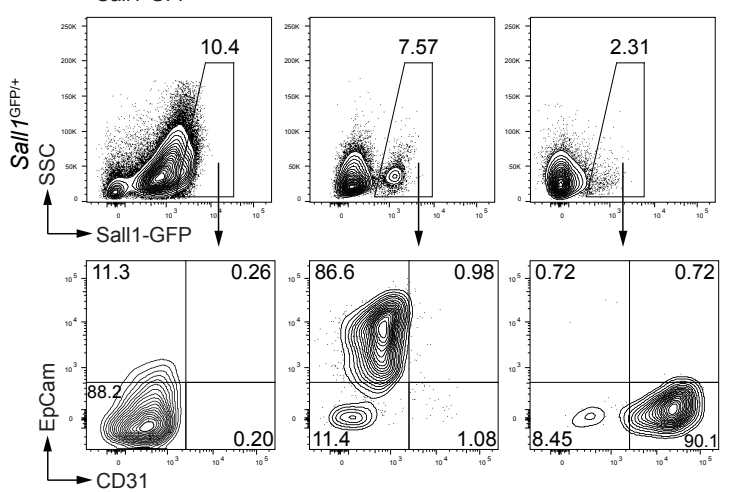

Figure 11 | Sall1 is expressed in a minor fraction of non-hematopoietic cells in kidney liver and heart. (a) Quantitative RT-PCR of Sall1 mRNA expression ( \pm SEM) of total tissue lysates of different organs. (Pooled data of at least 2 individual experiments, $n \geq 4, L N n=3$ ). Data were normalized to Po/2 expression. (b-c) Representative flow cytometry plots (b) and quantification of the frequency ( \pm SEM) (c) of GFP (Sall1) expression in kidney, liver and heart of Sall $1^{\mathrm{GFP} /+}$ and Sall $1^{+/+}$control mice (gated on CD45 cells). (Pooled data of 2-3 individual experiments, $n=5-6$ ). 
It was previously shown that Sall 1 is highly expressed by neuronal and glial progenitors in the CNS during embryogenesis, where it was implicated in cortical neurogenesis during CNS development ${ }^{91}$. To examine more carefully whether other CNS-resident cells apart from microglia express Sall1 in the adult, we analyzed the CNS of Sall $1^{\mathrm{GFP} / \mathrm{t}}$ mice by flow cytometry and immunohistochemistry (IHC). Unsupervised dimensionality reduction of the obtained flow cytometry data using the t-SNE algorithm overlaid with the GFP expression map showed that Sall1 is mainly expressed by microglia and that GFP expressing CD45- cells were scarce (Figure 12a). This observation was verified by thorough analysis of different nonhematopoietic populations, including astrocytes $\left(\mathrm{GLAST}^{+}\right)$, oligodendrocytes $\left(\mathrm{MOG}^{+}\right)$and endothelial cells $\left(\mathrm{CD} 31^{+}\right)$, in conventional two-dimensional flow cytometry plots, showing that only CNS-resident microglia express GFP (Figure 12b,c). Furthermore, within the adult CNS we did not detect notable GFP expression in CNS-resident cells apart from microglia (Iba- $1^{+}$) by $\mathrm{IHC}$ of brain sections from Sall $1^{\mathrm{GFP} /+}$ mice. This includes neuronal precursors (radial-glia-like stem cells, GFAP ${ }^{+}$or neuroblasts, $\left.\mathrm{DCX}^{+}\right)$, neurons $\left(\mathrm{NeuN}^{+}\right.$, Calbindin $\left.{ }^{+}\right)$or other non-neuronal cells such as astrocytes $\left(\mathrm{GFAP}^{+}, \mathrm{S} 100 \mathrm{~B}^{+}\right)$and oligodendrocytes $\left(\mathrm{Olig2}^{+}, \mathrm{MBP}^{+}\right.$) (Figure 12d-f). 
a

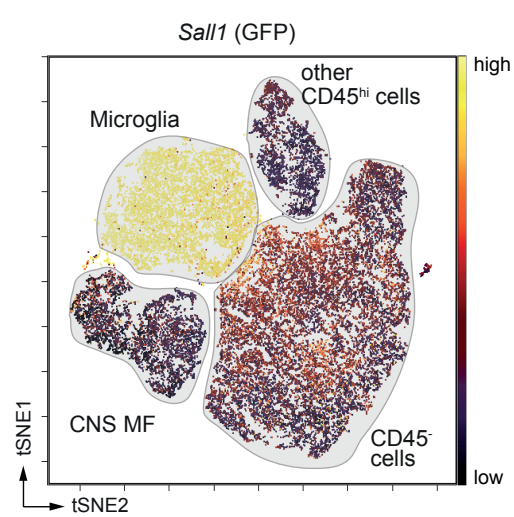

d
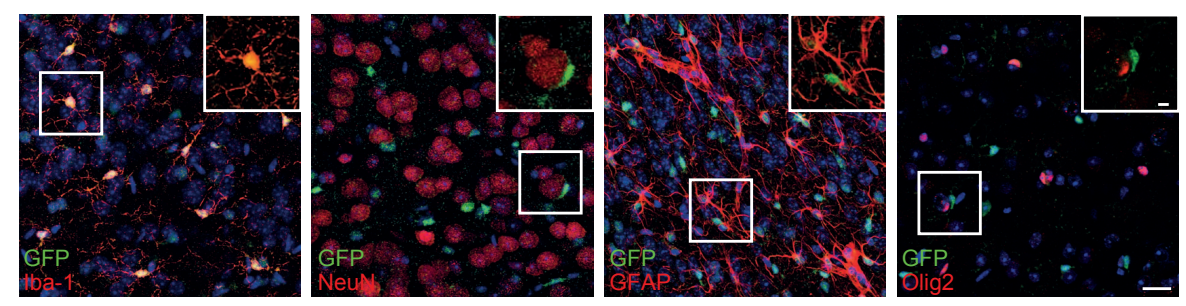

(1)
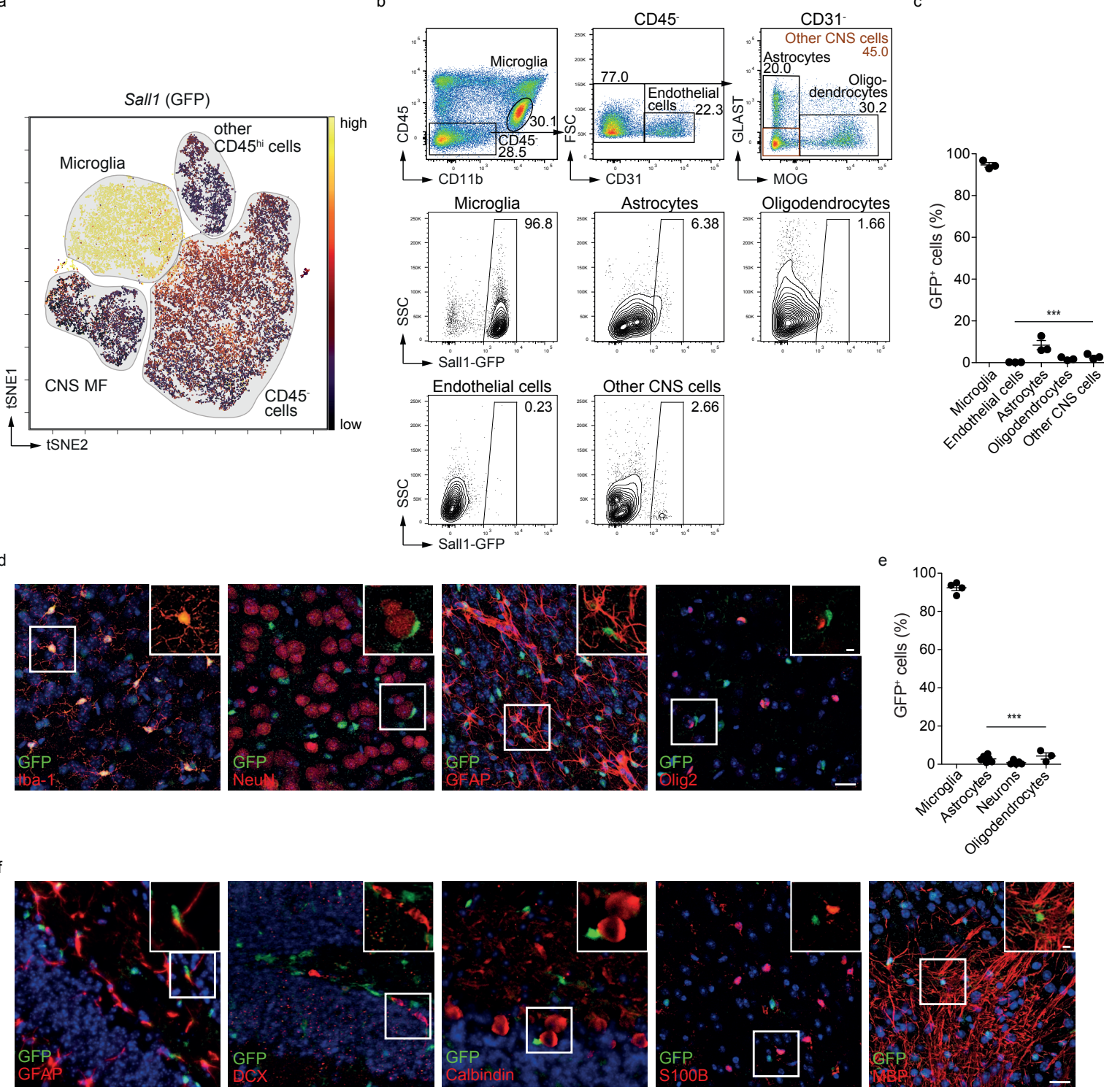

Figure 12 | Sall1 expression is specific to resident microglia within the CNS. (a) Annotated t-SNE map depicts populations of total CNS cells of Sall $1^{\mathrm{GFP} / \mathrm{H}}$ mice and color-coded overlay of Sall1 (GFP) expression. (b) Gating strategy of non-hematopoietic (CD45) CNS-resident cells and representative flow cytometry plots for their GFP (Sall1) expression in Sall $1^{\text {GFP/+ }}$ mice. (c) Quantification ( \pm SEM) of GFP $^{+}$cells by flow cytometry as shown in the gating strategy in b) in Sall $1^{\mathrm{GFP} / \mathrm{t}}$ mice $(\mathrm{n}=3)$. (d) Immunohistochemistry (IHC) of cortical brain sections of Sall ${ }^{\mathrm{GFP} / \mathrm{t}}$ mice for GFP (green), DAPI (blue) and either Iba-1 (microglia), NeuN (neurons), GFAP (astrocytes) or Olig2 (oligodendrocytes and their precursors) (red). Small images (without DAPI) display the magnification of the inset in the overview picture. (Scale bar: $20 \mu \mathrm{m}$ in the overview image and $5 \mu \mathrm{m}$ in the small images). (e) Quantification of d). Shown is the percentage of $\mathrm{GFP}^{+}$cells ( \pm SEM). Counted were 5-15 sections of 3-5 mice. (f) Immunohistochemistry of CNS sections of Sall $1^{\mathrm{GFP} /+}$ mice for GFP (green), DAPI (blue), and in red GFAP (radial-glia-like stem cells), DCX (neuroblasts), Calbindin (Purkinje neurons), S100B (astrocytes) and MBP (oligodendrocytes), respectively. Small images (without DAPI) display the magnification of the inset in the overview picture. (Scale bar: $20 \mu \mathrm{m}$ in the overview image and $5 \mu \mathrm{m}$ in the small images). One-way Anova, ${ }^{* * *} p<0.001$ (c, e). 
In order to secure the immunologically unique status of the CNS, specialized macrophage populations can be found at the boarder areas. Based on a recent publication by Goldmann et al. some of these macrophages, namely meningeal and perivascular macrophages, are embryonically derived and long-lived whereas choroid plexus macrophages are slowly repopulated by circulating monocytes ${ }^{52}$. To investigate whether these 'CNS-associated' macrophages are similar to microglia and also express Sall1 we quantitatively assayed their GFP expression in Sall $1^{\mathrm{GFP} /+}$ mice. As previously proposed ${ }^{52}$, we identified $\mathrm{CD}^{206^{+}}$non-parenchymal macrophages but also CD206- macrophages / dendritic cells by flow cytometry. While macrophages residing in the choroid plexus were essentially negative for GFP, we found approximately $5 \%$ of other CNS-associated macrophages to be positive (Figure 13a-f). To further visualize GFP-expressing cells in the CNS of Sall ${ }^{\mathrm{GFP} /+}$ mice, we used unsupervised dimensionality reduction (t-SNE) of our single cell flow cytometry data, which also revealed that in the CNS, microglia are the prevailing population expressing Sall1 while GFP was detected only in a minor fraction of 'non-microglial' CNS cells (Figure 13b,c). In addition, we visualized these macrophages based on their location and commonly used macrophage markers in $\mathrm{HC}$, showing only little co-localization with GFP-labeled cells (Figure 13e). Of note, this is a major advantage over the commonly used $C \times 3 c r 1^{\mathrm{GFP} /+}$ reporter mouse which targets not only microglia but also the vast majority of 'CNSassociated' myeloid cells (Figure 13f) ${ }^{98}$. Taken together these data demonstrate, within the adult CNS and within the hematopoietic system, Sall1 expression is restricted to microglia. 
a
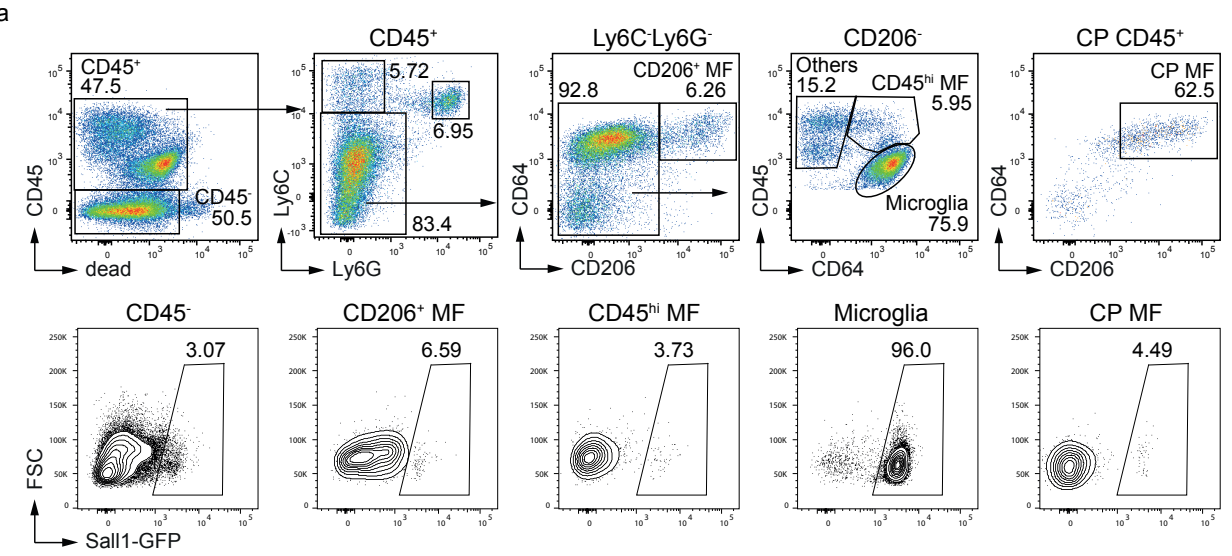

b
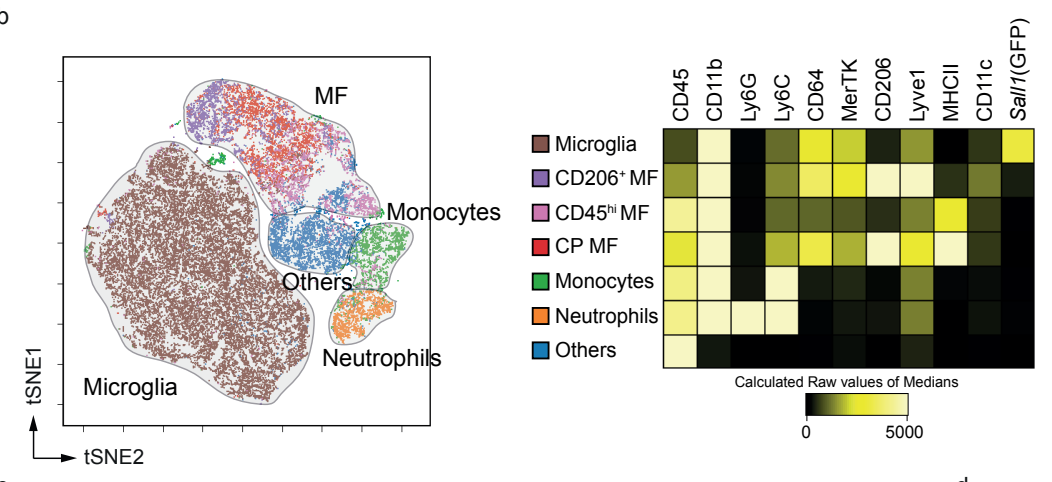

c

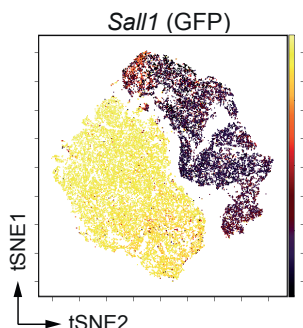

CD45
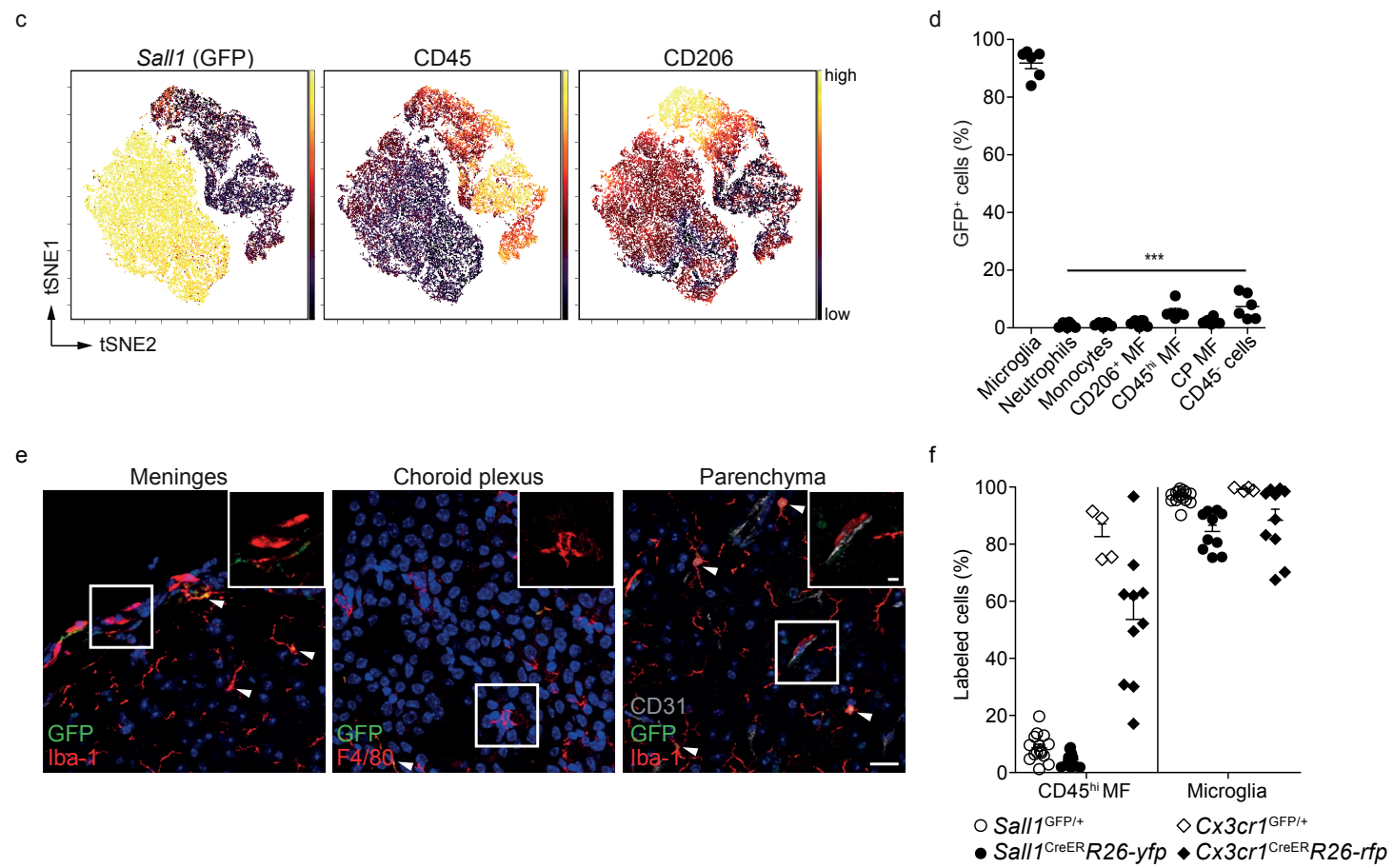

Figure 13 | Sall1 is not expressed by other CNS-associated myeloid cells. (a) Gating strategy of CNSresident myeloid cells and separately isolated choroid plexus (CP) cells. Representative Flow cytometry plots display frequencies of $\mathrm{GFP}^{+}$cells in Sall $1^{\mathrm{GFP} /+}$ mice. MF: Macrophage. (b) Annotated t-SNE map and expression of markers in the identified populations of $\mathrm{CD} 45^{+} \mathrm{CNS}$ cells of Sall $1^{\mathrm{GFP} /+}$ mice. (c) as in b), t-SNE map depicts color-coded overlay of Sall1 (GFP), CD45 and CD206 expression of CD45 ${ }^{+}$cells of Sall $1^{\text {GFP/+ }}$ mice. (d) Quantification ( \pm SEM) of $\mathrm{GFP}^{+}$cells by flow cytometry as shown in the gating strategy in a) $(n=6)$. One-way Anova, ${ }^{* * *} p<0.001$. 
(e) Immunohistochemistry of CNS sections of Sall $1^{\mathrm{GFP} /+}$ mice for GFP (green), DAPI (blue), Iba-1 or F4/80 (microglia and CNS-MF, red) and CD31 (endothelial cells, gray), respectively. Small images without DAPI display the magnification of the inset in the overview picture. Representative pictures are shown. $(n=2-4)$ (Scale bar: $20 \mu \mathrm{m}$ in the overview image and $5 \mu \mathrm{m}$ in the small images, arrowheads indicate lba-1 and GFP or F4/80 and GFP double-positive microglia). (f) Frequency ( \pm SEM) of GFP $^{+}$microglia and CD45 ${ }^{\text {hi }}$ MF of Sall $1^{\mathrm{GFP} /+}$ and $\mathrm{C} \times 3 \mathrm{cr} 1^{\mathrm{GFP} /+}$ reporter mice and of $\mathrm{YFP}^{+}$and $\mathrm{RFP}^{+}$microglia and CD45 ${ }^{\mathrm{hi}}$ MF in tamoxifen treated Sall $1^{\text {CreER }} R 26$-yfp and $\mathrm{C} \times 3 \mathrm{cr}{ }^{\mathrm{CreER}} \mathrm{R} 26$-rfp mice, respectively. (Pooled data of at least 2 individual experiments, $\mathrm{n}=4-13)$. Cells were pregated on CD45 ${ }^{+} \mathrm{CD} 11 \mathrm{~b}^{+} \mathrm{F} 4 / 80^{+} \mathrm{Ly}_{6 C^{-} L y 6 G^{-}}$.

\subsection{The CNS environment alone does not imprint Sall1 expression}

Under physiological conditions, microglia are not replaced by circulating precursors. However upon experimental microglia depletion, the empty microglia niche is reconstituted rapidly. The origin of the newly formed microglia/microglialike cells is a matter of some debate. Recent reports suggest that the ontogeny of newly emerging microglia/microglia-like cells is dependent on the experimental setup and that they can be derived either from circulating precursors (BM-derived) in a BM transplantation setting or from an internal pool of CNS-resident cells ${ }^{51,99-}$ ${ }^{101}$. Given that Sall1 represents a microglia signature gene, we addressed whether newly repopulated microglia/microglia-like cells also expressed Sall1. As a model for CNS-internal repopulation of microglia upon depletion, we treated Sall $1^{\mathrm{GFP} /+}$ and WT mice with Csf-1R inhibitors (PLX5622) for 2 weeks. As recently described by Elmore et al., this treatment leads to a rapid depletion of microglia, which are fully repopulated by the remaining CNS-resident microglia within one week after treatment (Figure 14a) ${ }^{101}$. We found the few remaining microglia of Sall $1^{\mathrm{GFP} /+}$ mice to be $\mathrm{GFP}^{+} 1$ day after PLX5622-treatment (Figure 14b-d). Two weeks after discontinuing PLX5622-treatment, newly formed 'microglia' expressed Sall1 similarly to the untreated control mice (Figure 14b-d). On the other hand, when we used congenic Sall1 ${ }^{\mathrm{GFP} /+} \mathrm{BM}$ chimeras $\left(\right.$ Sall ${ }^{\mathrm{GFP} /+}(\mathrm{CD} 45.1) \rightarrow \mathrm{WT}(\mathrm{CD} 45.2)$ ), we found that after exposure to Csf-1R inhibitors, newly repopulated microglia-like cells were BM-derived (CD45.1) and did not express Sall1 even 4 weeks after repopulation (Figure 14e,f). Also in the recently described $C \times 3 c r 1^{\mathrm{CreER}} i D T R$ model combined with BM chimerism ${ }^{100}$, we could show that the newly BM-derived microglia-like cells failed to express Sall1 (Figure 14g,h ). 
Taken together, new microglia-like cells originated from BM-derived cells after microglial depletion do not initiate Sall1 transcription, whereas new microglia derived from a CNS-resident pool retain Sall1 expression. Previous reports also suggested that BM-derived microglia-like cells have an altered genetic signature compared to yolk sac or internally-derived microglia upon depletion, whereas the genetic profile of the latter two remained largely identical including the expression of Sall ${ }^{100}$. Our data indicate that Sall1 is a marker for bona fide microglia and can be used to discriminate the ontogenically different embryonically-derived microglia from microglia-like cells derived from the BM. 


\section{Results}

a
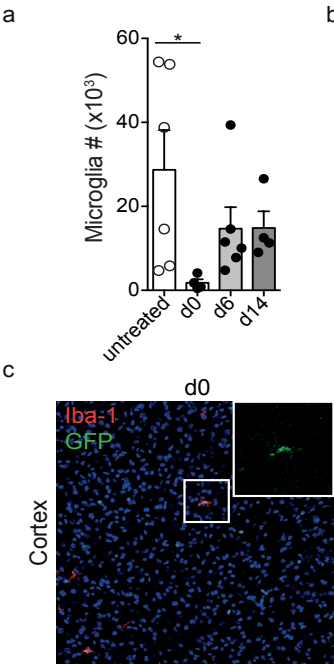

e

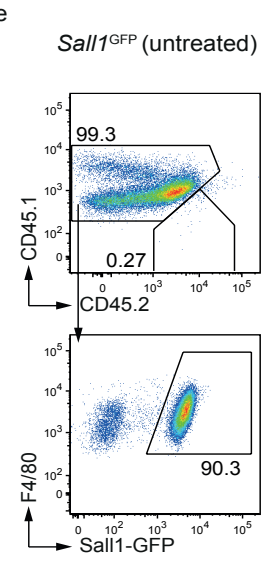

g 11

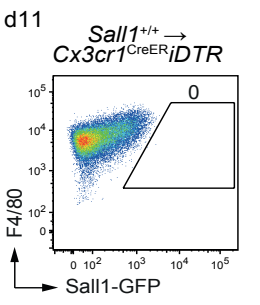

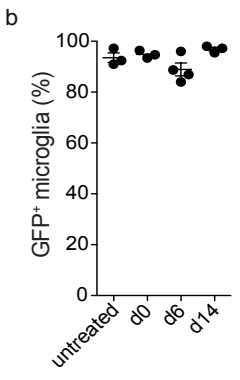
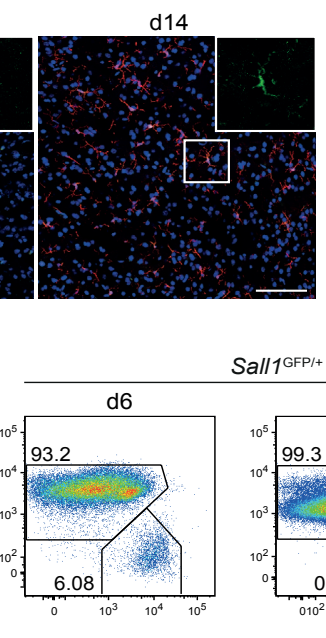
$\mathrm{Sall1}$ GFP/+ $^{\mathrm{G}} \mathrm{WT}(\mathrm{PLX} 5622)$
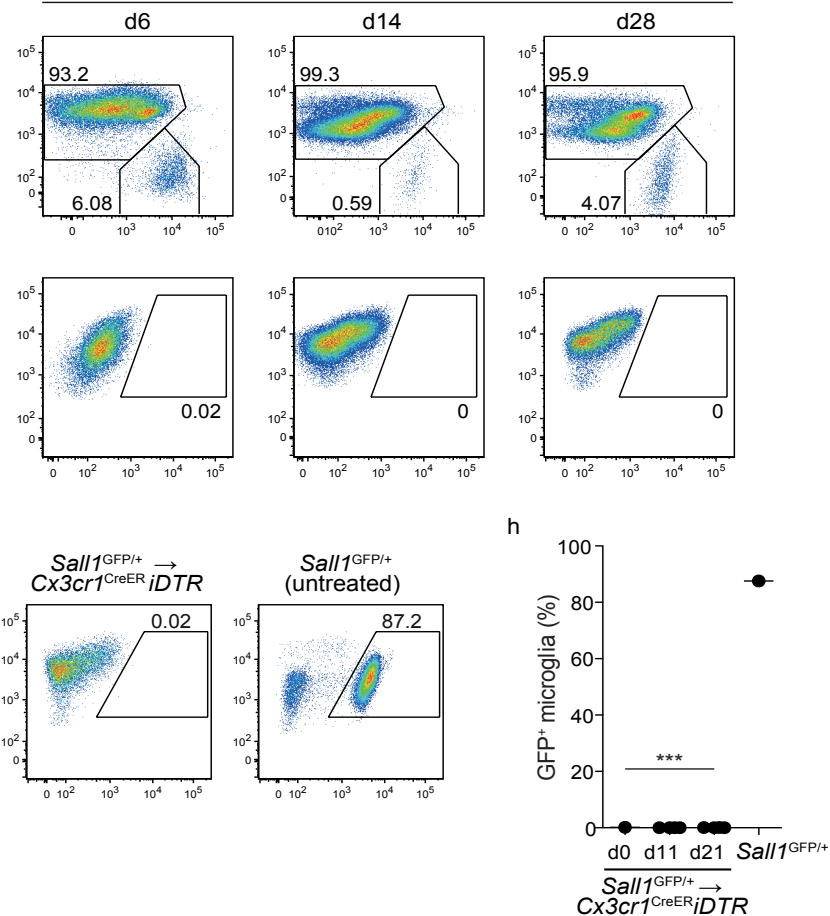
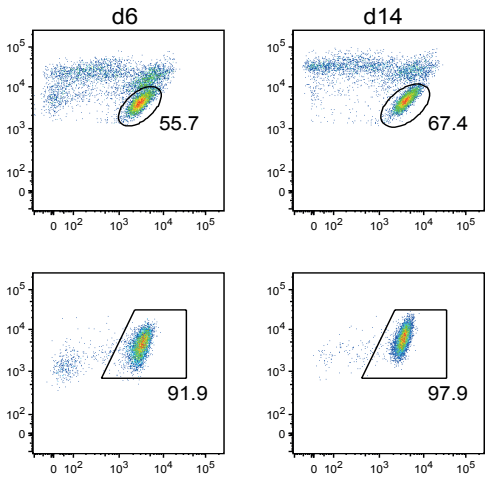

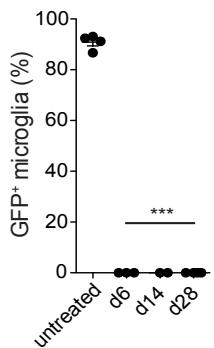

Figure 14 | BM-derived 'microglia-like' cells do not express Sall1. (a-d) Sall1 ${ }^{\mathrm{GFP} /+}$ mice and Sall1 ${ }^{+/+}$ littermates were treated with PLX5622 food for 2 weeks. Bar graphs (a) show microglia numbers at d0, d6 and d14 after treatment stop or in untreated mice. Scatter plots (b) and representative IHC images (c) and flow cytometry plots (d) (pre-gated on $\mathrm{CD}_{4} 5^{+}$cells) show the frequency ( \pm SEM) of GFP ${ }^{+}$microglia at d0, d6 and d14 after treatment stop or in untreated mice. (Pooled data of 2 independent experiments, $n=3-4)$. (Scale bar: $100 \mu \mathrm{m})$. (e-f) Representative flow cytometry plots (e) and scatter graphs (f) show frequency ( \pm SEM) of GFP ${ }^{+}$ microglia (pre-gated on CD11 $\mathrm{b}^{+} \mathrm{F} 4 / 80^{+}$Ly6C $^{-}$Ly6G $^{-}$cells) in congenic Sall ${ }^{\mathrm{GFP} /+}$ (CD45.1) $\rightarrow$ WT (CD45.2) BM chimeras at d6, d14 and d28 after PLX5622 treatment (2 weeks) and untreated Sall1 ${ }^{\mathrm{GFP} /+}$ (CD45.1) controls. (Pooled data of 2 independent experiments, $n=2-3$ for Sall $1^{\mathrm{GFP} /+} \rightarrow$ WT BM chimeric mice and $n=4$ for Sall $1^{\mathrm{GFP} /+}$ control mice). (g-h) Representative flow cytometry plots (g) and scatter plots (h) display the frequency $\left( \pm\right.$ SEM) of GFP ${ }^{+}$microglia of tamoxifen/DTx-treated Sall $1^{+/+}\left(\right.$CD45.1) $\rightarrow C x 3 c r 1^{\text {CreER }}$ iDTR (CD45.2) or Sall $1^{\mathrm{GFP} /+}(\mathrm{CD} 45.1) \rightarrow C \times 3 c r 1^{\mathrm{CreER}}$ iDTR (CD45.2) BM chimeras (pre-gated on CD11 $\mathrm{b}^{+} \mathrm{F} 4 / 80^{+} \mathrm{CD} 45.1^{\mathrm{lo}} \mathrm{Ly}_{6 \mathrm{C}^{-}}$ Ly6G ${ }^{-}$cells) on d0, d11 and d21 after treatment. Untreated Sall $1^{\mathrm{GFP} /+}$ mice served as a GFP control. (Pooled data of 2 individual experiments, $n=4$ for $\mathrm{d} 11$ and $d 21, n=1$ for $d 0$ and untreated Sall $1^{\mathrm{GFP} /+}$ ). One-way Anova, ${ }^{*} p<0.05,{ }^{* * *} p<0.001(a, f, h)$. 
Similarly in the context of neuroinflammation, it has been reported that microglia become activated, yet maintain a signature distinct from invading myeloid cells ${ }^{50}$. Thus, we investigated whether CNS-infiltrating myeloid cells would also acquire Sall1 expression after recruitment to the brain. The neuroinflammatory model of experimental autoimmune encephalomyelitis (EAE), a preclinical model for multiple sclerosis, is characterized by massive infiltration of monocytes and their subsequent differentiation into monocyte-derived cells such as monocyte-derived DCs or inflammatory macrophages ${ }^{102,103}$. In order to distinguish infiltrating immune cells from resident irradiation-resistant microglia, we generated congenic (CD45.1 or CD45.2) Sall $1^{\mathrm{GFP} /+} \mathrm{BM}$ chimeras (Sall $1^{\mathrm{GFP} /+} \rightarrow \mathrm{WT}$ and $\mathrm{WT} \rightarrow$ Sall $1^{\mathrm{GFP} /+}$ ) and immunized them s.c. with $\mathrm{MOG}_{35-55} / \mathrm{CFA}$. At peak disease, microglia continued to express Sall1, while CNS-invading myeloid cells including neutrophils and monocyte-derived cells did not (Figure 15a,b). Similar results were observed using Sall $1^{\mathrm{GFP} /+}$ mice without BM chimerism (Figure 15c). As in parts shown previously ${ }^{84}$, this demonstrates that Sall1 expression is inherent to resident microglia and that the CNS tissue environment does not induce Sall1 expression on myeloid populations under inflammatory conditions.
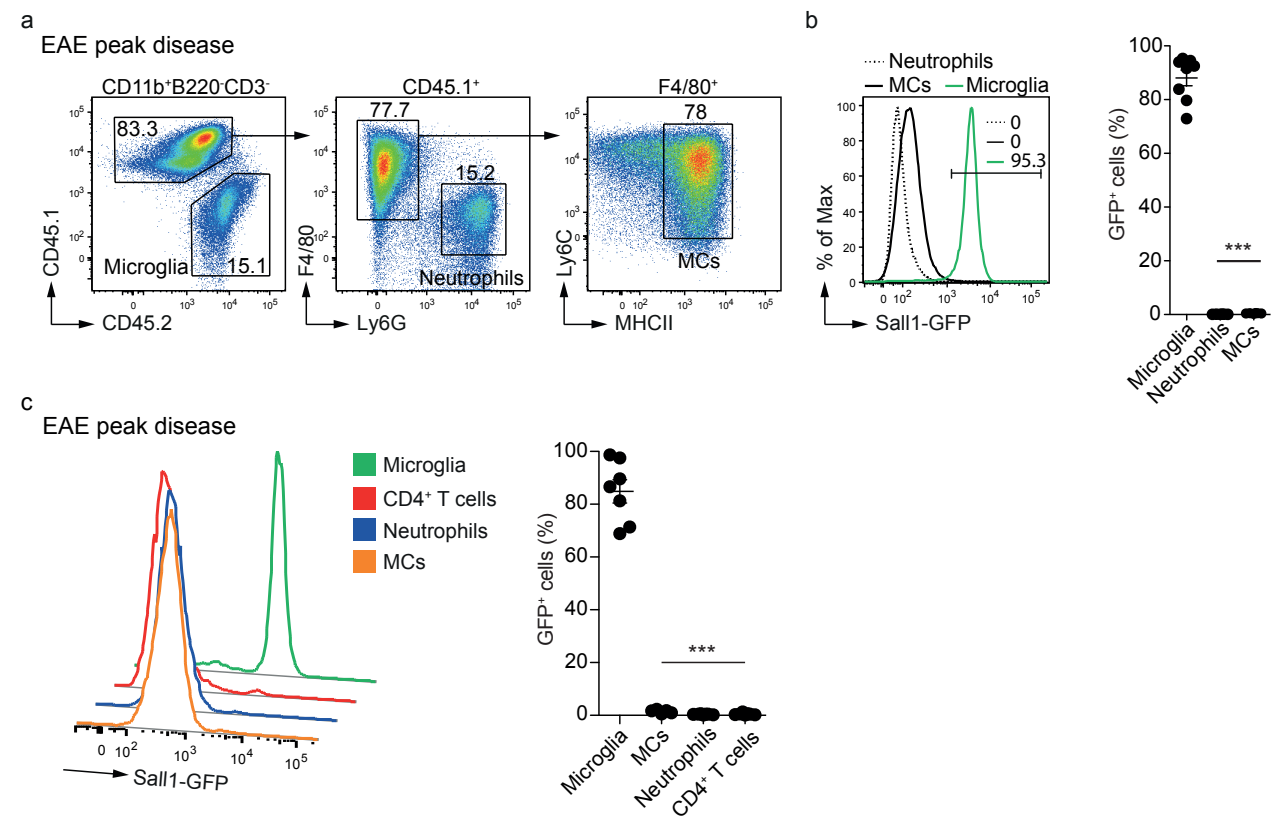

Figure 15 | CNS-infiltrating myeloid cells do not express Sall1. 


\section{Results}

(a-b) CNS of Sall $1^{\mathrm{GFP} /+}(\mathrm{CD} 45.1) \rightarrow \mathrm{WT}(\mathrm{CD} 45.2)$ or $\mathrm{WT}(\mathrm{CD} 45.2) \rightarrow$ Sall $^{\mathrm{GFP} /+}(\mathrm{CD} 45.1)$ BM chimeras were analyzed for GFP expression at peak disease of $\mathrm{MOG}_{35-55} / \mathrm{CFA}$ induced EAE. (a) Gating strategy is shown as an example of a Sall ${ }^{\mathrm{GFP} /+}(\mathrm{CD} 45.1) \rightarrow \mathrm{WT}(\mathrm{CD} 45.2) \mathrm{BM}$ chimeric mouse. (b) Histogram depicts the percentage of GFP expression of MCs and neutrophils (of Sall $1^{\mathrm{GFP} /+}$ (CD45.1) $\rightarrow$ WT(CD45.2) BM chimeras) and microglia (of WT(CD45.1) $\rightarrow$ Sall $1^{\text {GFP/+ }}$ (CD45.2) BM chimeras) as gated in a) and graph shows the quantification ( \pm SEM). (Pooled data of two individual experiments, $n=6-8$ ). (c) Representative histograms and quantification ( \pm SEM) of GFP (Sall1) expression in MCs (gated on CD45 ${ }^{\mathrm{hi}} \mathrm{CD} 11 \mathrm{~b}^{+} \mathrm{CD} 11 \mathrm{c}^{+} \mathrm{MHCI}{ }^{+} \mathrm{Ly} 6 \mathrm{C}^{+}$), neutrophils (gated on

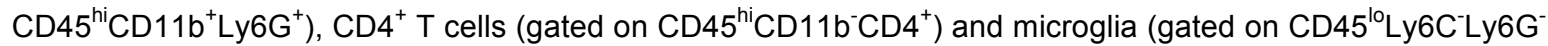

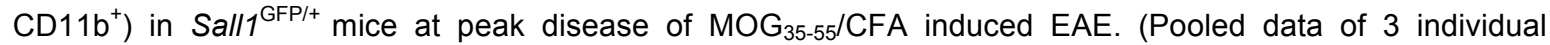
experiments, $n=5-7)$. One-way Anova, ${ }^{* *} \mathrm{p}<0.001(\mathrm{~b}, \mathrm{c})$. 


\subsection{Csf1r and Tgfbr2 have different contributions to microglia homeostasis}

To determine the utility of the Sall 1 promoter for conditional gene targeting in microglia, we used Sall1 ${ }^{\text {CreER }}$ mice, which express the tamoxifen-inducible Crerecombinase under the Sall1 promoter ${ }^{104}$. We found the Sall1 ${ }^{\text {CreER }}$ strain to efficiently recombine in microglia $(>90 \%)$ when crossed to the reporter/fate-map strain (R26-yfp), whereas no other hematopoietic cells or other CNS-resident cells were labeled (Figure 13f, Figure 16a and data not shown).

Csf-1R signaling is critical for the embryonic development of microglia ${ }^{46}$. As described above and suggested previously, in the adult, Csf-1R signaling blockade by Csf-1R kinase inhibitors led to the depletion of microglia ${ }^{101}$. However Csf-1R inhibitor treatment is not only specific for Csf-1R but also blocks other tyrosine kinases such as FLT3, PDGFR and KIT ${ }^{51}$. We could show that upon tamoxifen treatment (Figure 16a), adult microglia were rapidly depleted in Sall $1^{\mathrm{CreER}} \mathrm{Csf1} \mathrm{r}^{\mathrm{fl} / \mathrm{fl}}$ mice, with varying efficiencies between $70-90 \%$ in different brain regions (Figure 16c-g). As described in other microglia depletion models ${ }^{51}$, also in this model they were repopulated within 7 days (Figure 16d,e). To analyze whether the untargeted, remaining microglia could replenish the empty microglia niche, we examined their proliferation by $\mathrm{IHC}$ and flow cytometry and found that more than half of the remaining microglia were $\mathrm{Ki}^{+}$and thus highly proliferative (Figure $16 \mathrm{~g}, \mathrm{~h}$ ). Therefore, in addition to being critical for their development ${ }^{46}$, this specific, inducible approach demonstrates that Csf-1R signaling in microglia is crucial for their homeostasis in the adult brain. 


\section{Results}

a
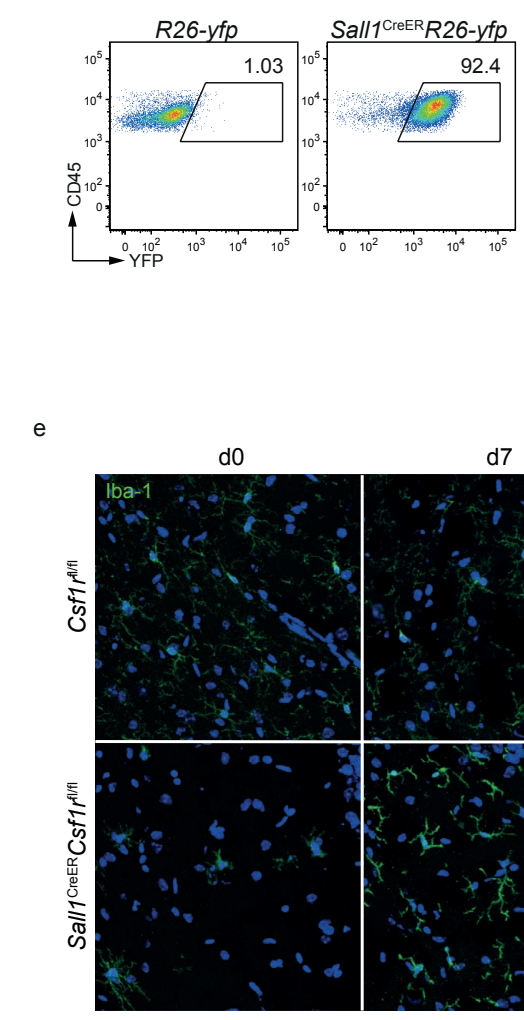

g

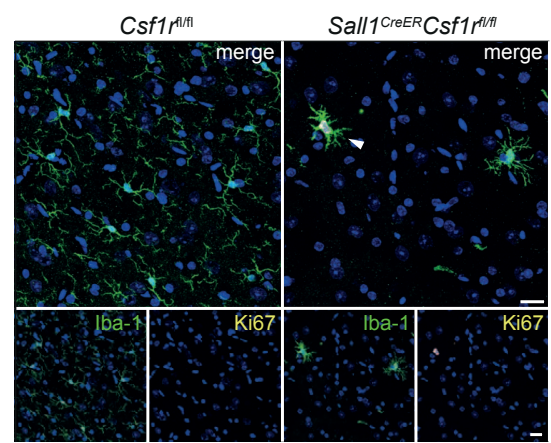

b

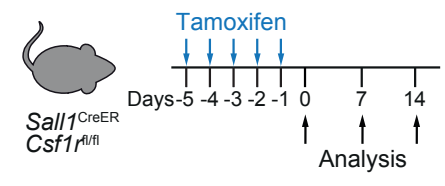

c do

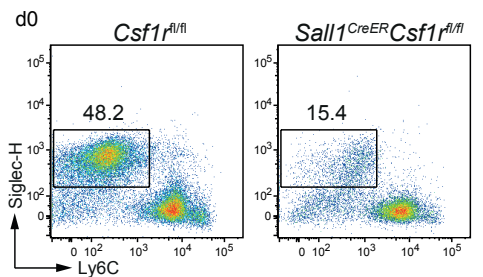

d

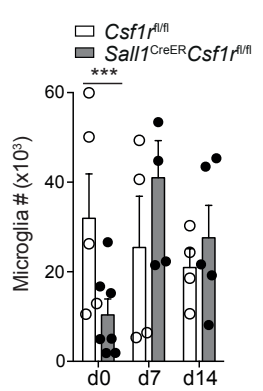

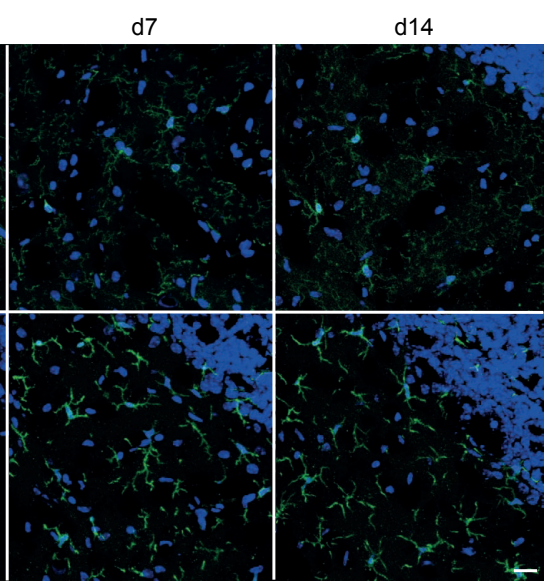

d0

h
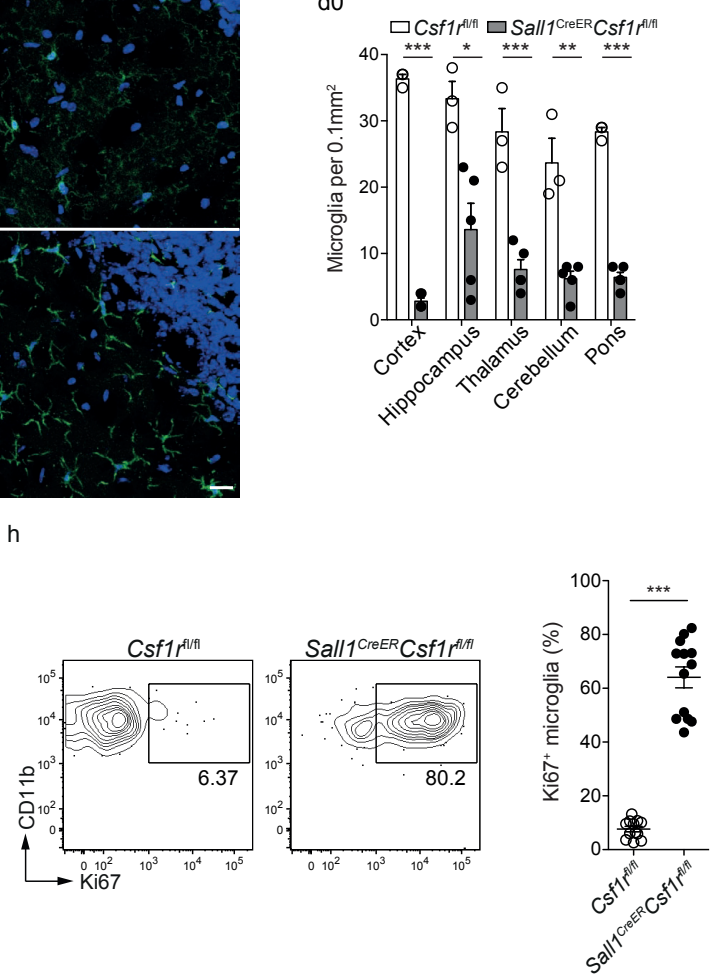

Figure 16 | Csf-1R signaling is crucial for microglia maintenance. (a) Flow cytometry plots display the frequency of $\mathrm{YFP}^{+}$microglia (pre-gated on CD45 ${ }^{\mathrm{lo}} \mathrm{Ly} 6 \mathrm{G}^{-} \mathrm{Ly}_{6 \mathrm{C}} \mathrm{CD}^{-} 11 \mathrm{~b}^{+}$) of Sall $1^{\mathrm{CreER}} R 26$-yfp mice and R26-yfp control littermates on $\mathrm{d} 3$ after tamoxifen treatment. (Representative plots of at least 5 similar individual experiments, $\mathrm{n}=10$ ). (b) Scheme of the experimental setup for c-h. Sall1 ${ }^{\mathrm{CreER}} \mathrm{Csf1} \mathrm{r}^{\mathrm{fl} / \mathrm{fl}}$ mice and Csf1 $\mathrm{r}^{\mathrm{fl} / \mathrm{fl}}$ littermates were treated with tamoxifen $(2.5 \mathrm{mg}$ i.p.) for 5 consecutive days and analyzed at different time points after treatment. (c-d) Representative flow cytometry plots (c) show the percentage of microglia (Siglec$\mathrm{H}^{+}$) (pre-gated on $\mathrm{CD} 45^{+} \mathrm{CD} 11 \mathrm{~b}^{+}$cells) and graph (d) displays the total cell number ( $\pm \mathrm{SEM}$ ). (Pooled data of 2 independent experiments, $n=4-7$ ). (e) $\mathrm{IHC}$ of cortical brain sections of tamoxifen treated Sall1 ${ }^{\mathrm{CreER}}{ }^{\mathrm{Csf}} 1 \mathrm{r}^{\mathrm{f} / \mathrm{fl}}$ and Csf1 $r^{\text {fl/fl }}$ mice for lba-1 (green, microglia) and DAPI (blue) at d0, d7 and d14 after tamoxifen treatment. (Scale bar: $20 \mu \mathrm{m})$. (f) Graph ( \pm SEM) shows microglia counts in different brain areas at d0 after tamoxifen treatment. ( $n=3-5$ sections of 2 mice). (g) Representative IHC images for Iba-1 (green), DAPI (blue) and Ki67 (yellow) in the thalamus at d0 after tamoxifen treatment. (Scale bar: $20 \mu \mathrm{m}$ ). (h) Representative flow cytometry plots (pregated on $C D 45^{10} \mathrm{CD} 11 \mathrm{~b}^{+}$microglia) and graph $\left( \pm\right.$SEM) depict the percentage of Ki67 ${ }^{+}$microglia at d0 after tamoxifen treatment. (Pooled data of 3 independent experiments, $n=12-13$ ). Unpaired $t$-test, ${ }^{* * *} p<0.001(d$, h). 
A recent report suggested a TGF- $\beta$-dependent gene signature in microglia and claimed that TGF- $\beta$ is critical for the development and maintenance of microglia ${ }^{50}$. Utilizing Sall1 ${ }^{\mathrm{CreER}}$ mice, we wanted to confirm the importance of TGF- $\beta$ R signaling in adult microglia. To do so, we crossed the Sall $1^{\text {CreER }}$ mice to the Tgfbr $2^{\text {fl/fl }}$ mice and exposed adult mice to tamoxifen to delete TGF- $\beta$ R on microglia (Figure 17a). Recombination frequency and deletion of Tgfbr2 was verified by qPCR (Figure 17b). The deletion of Tgfbr2 in microglia of tamoxifen treated Sall $1^{\mathrm{CreER}} \mathrm{Tgfbr} 2^{\mathrm{fl} / \mathrm{fl}}$ mice led to a moderate increase in microglial cell numbers on $\mathrm{d} 6$ after treatment, (Figure 17c). Three days after tamoxifen treatment, microglia lacking TGF- $\beta R$ exhibited altered cell morphology including shorter, thicker dendrites and enhanced Iba-1 immunoreactivity characteristically seen in reactive microglia (Figure 17d). Furthermore we observed an increased number of proliferating $\left(\mathrm{Ki} 67^{+}\right)$microglia, which could explain the elevated microglia numbers (Figure 17d). Also by flow cytometry, we could show that TGF- $\beta$ R-deficient microglia present an altered surface phenotype by up-regulation of $\mathrm{CD} 45, \mathrm{~F} 4 / 80, \mathrm{MHCll}$ and intermediate levels of CD11c and Siglec-1 (Figure 17e,f) ${ }^{105}$. Of note, the marker Siglec-H, which was shown to be expressed by microglia but not by other tissue macrophages ${ }^{32}$, was down-regulated upon deletion of TGF- $\beta R$ on microglia.

To exclude the possibility that the CD $45^{\text {int-hi }} \mathrm{F} 4 / 80^{\text {hi }}$ population, which we observed after deletion of microglial Tgfbr2, was derived from circulating monocytes, we crossed the Sall $1^{\mathrm{CreER}} T g f b r 2^{\mathrm{flflf}}$ mice to the reporter/fate-map strain R26-yfp (Sall $1^{\mathrm{CreER}}$ Tgfbr2 $2^{\mathrm{fl} / \mathrm{fl}} R 26-\mathrm{yfp}$ ). Upon tamoxifen treatment, $\mathrm{YFP}^{+}$cells in

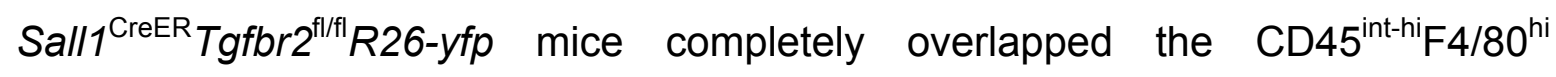
population, whereas other myeloid cells in the CNS or blood were negative for YFP (Figure 17g,h and data not shown). This demonstrates that CD $45^{\text {int-hi }} \mathrm{F} 4 / 80^{\text {hi }}$ cells were indeed bona fide microglia, which have up-regulated CD45. In addition, Tgfbr2-deficient microglia displayed elevated expression levels of II1, Tnfa and Cxc/10 similar to microglia isolated from LPS-challenged mice, which we used as a positive control for reactive microglia (Figure 17i). Taken together these results show that TGF- $\beta$ signaling in microglia is not crucial for their survival but is essential to keep them in a non-reactive state. 


\section{Results}

a

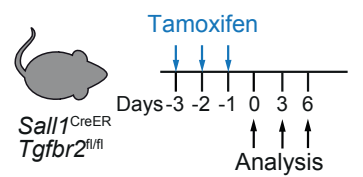

d
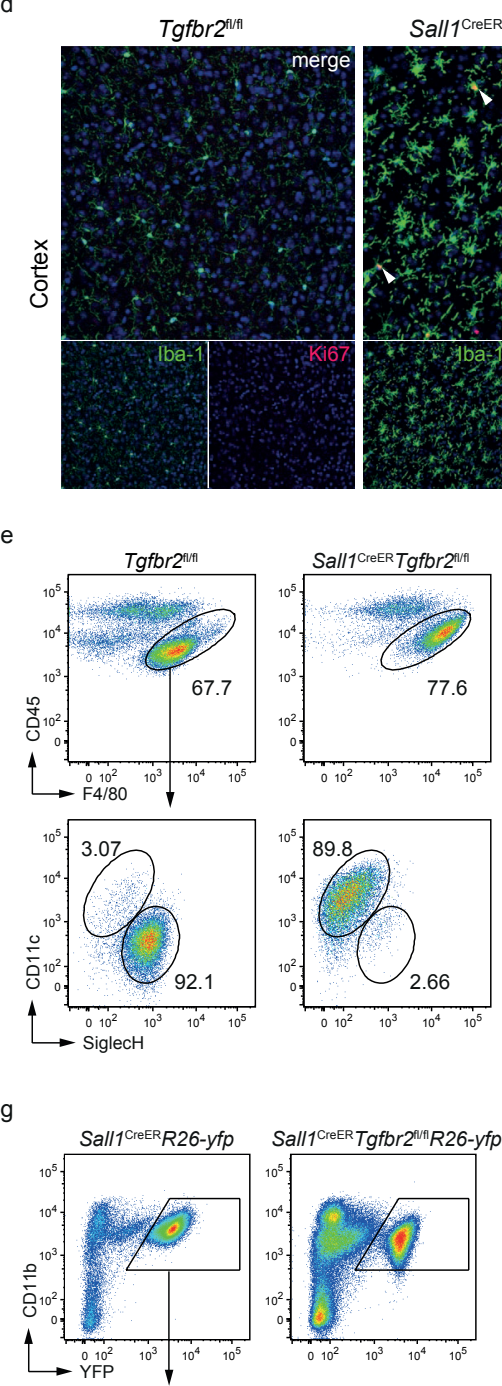

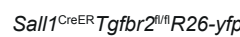
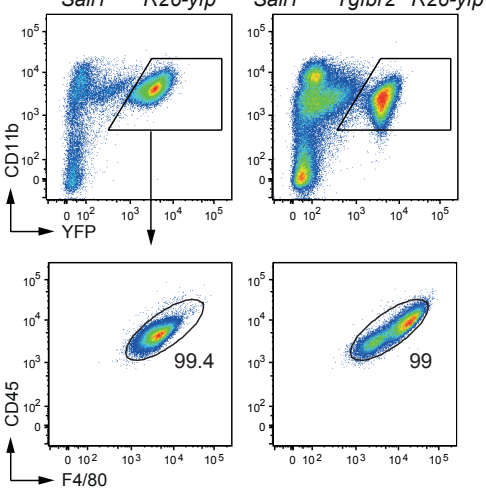

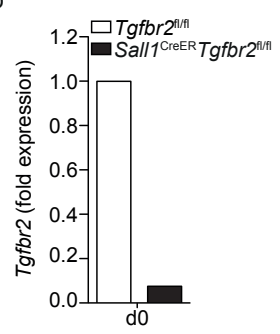

c

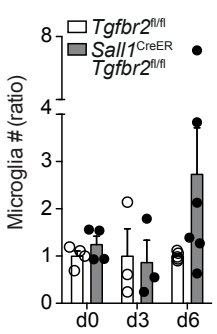

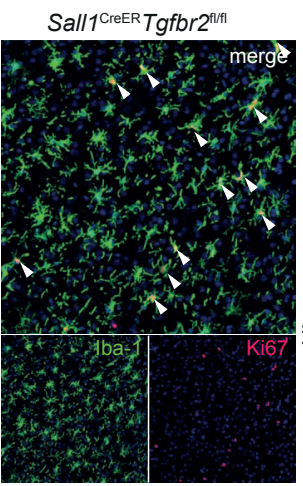

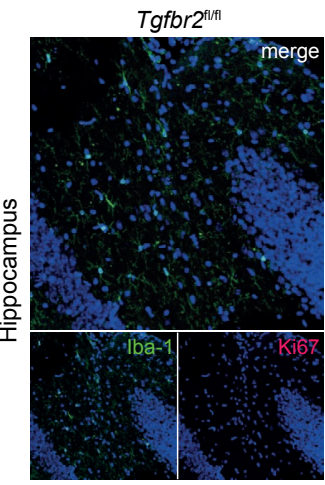

Sall/ ${ }^{\text {CreER }} \operatorname{Tgfbr}^{\mathrm{fl} / \mathrm{fl}}$
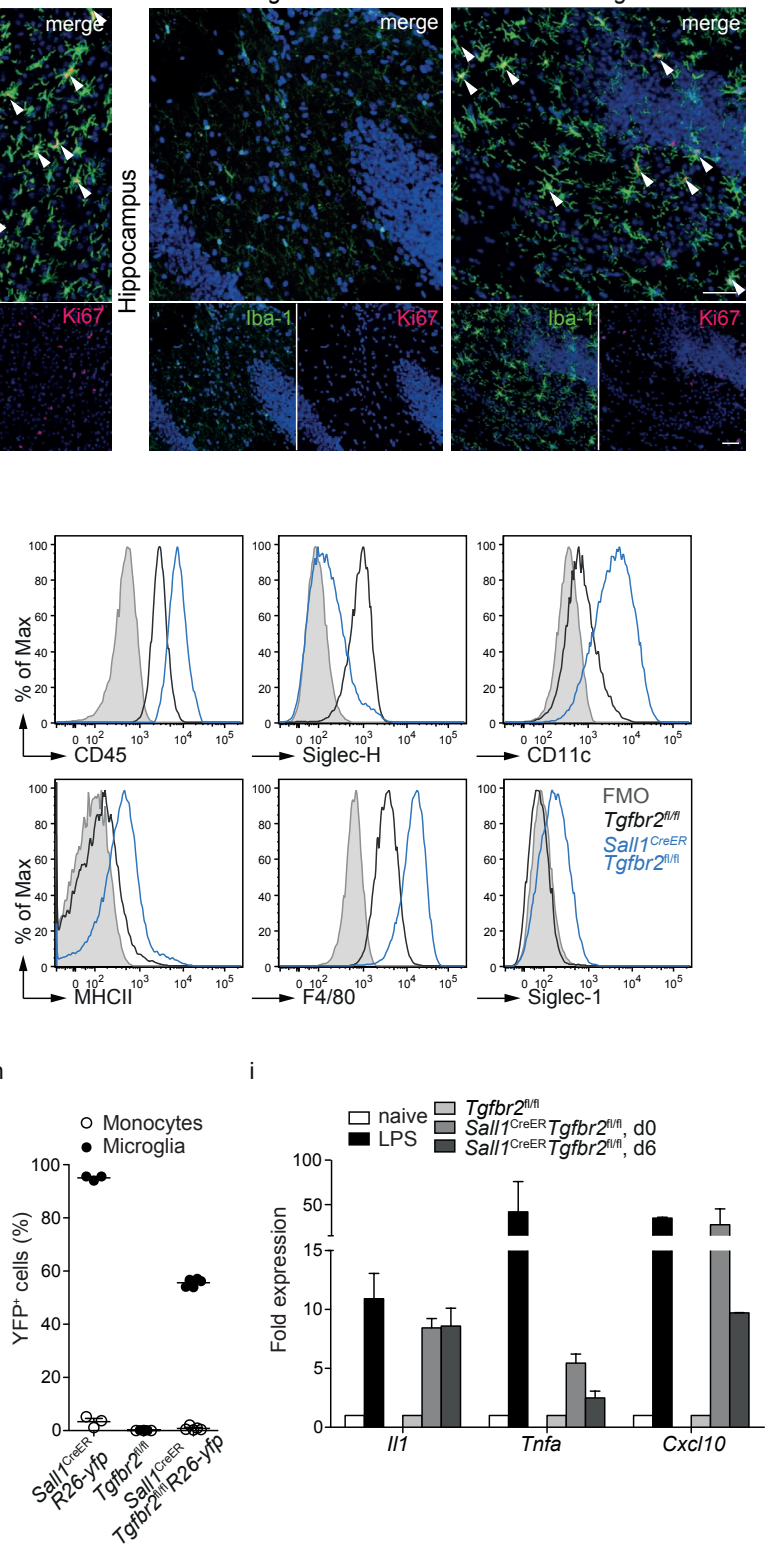

Figure 17 | TGF- $\beta R$ signaling maintains 'resting' microglia phenotype. (a) Scheme of the experimental

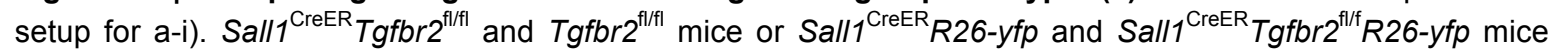
were treated with tamoxifen (2.5 mg i.p.) on three consecutive days and were analyzed at d0, d3 or d6 after treatment. (b) Quantitative qPCR of Tgfbr2 mRNA expression of microglia sorted from Sall ${ }^{\text {CreER }}{ }^{T g f b r} 2^{\mathrm{fl} / \mathrm{fl}}$ and $T g f b r 2^{f / f f l}$ mice on d0 after tamoxifen treatment. Data were normalized to Pol2 expression (pooled sample of 3 4 mice). (c) Quantification ( \pm SEM) of microglia numbers (assessed by flow cytometry analysis gated as in upper row of e) on $\mathrm{d} 0, \mathrm{~d} 3$ and $\mathrm{d} 6$ after tamoxifen treatment. Numbers are displayed as ratios to control $\left(T g f b r 2^{\mathrm{fl} / \mathrm{fl}}\right)$ mice. (Pooled data of 2 individual experiments, $n=3-6$.) 
(d) Representative IHC images of CNS sections of Sall ${ }^{\mathrm{CreER}}{ }^{\mathrm{Tgfb}} 2^{\mathrm{fl} / \mathrm{fl}}$ and $\mathrm{Tgfbr} 2^{\mathrm{fl} / \mathrm{fl}}$ mice on d3 after discontinuing treatment for Iba-1 (green), Ki67 (red) and DAPI (blue). Arrowheads indicate lba-1 and Ki67 double-positive cells. (Scale bar: $50 \mu \mathrm{m}, \mathrm{n}=1$ - 2). (e) Representative flow cytometry plots show frequency of microglia (pre-gated on $\mathrm{CD}_{4} 5^{+} \mathrm{Ly}_{6 C^{-}{ }^{-} \text {Ly6G }}{ }^{-}$cells) and expression of Siglec-H vs. CD11C on d6 after tamoxifen treatment. (2 individual experiments, $n=5-6$ ). (f) Histograms display the expression of different surface markers vs. FMO on microglia (gated as in upper row of e) of Sall1 ${ }^{\mathrm{CreER}} \mathrm{Tgfbr}^{\mathrm{fl} / \mathrm{fl}}$ and $\mathrm{Tgfbr}^{\mathrm{fl} / \mathrm{fl}}$ mice at d6 after tamoxifen treatment. (Plots are representatives of at least 2 individual experiments, $\mathrm{n} \geq 2$ ). (g-h) CNS of

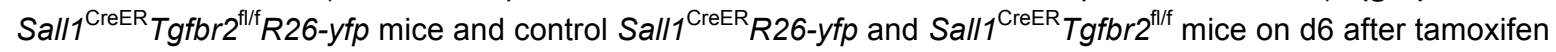
treatment. (g) Representative flow cytometry plots show YFP ${ }^{+}$cells (pre-gated on CD45 ${ }^{+}$cells). (h) Scatter plots show frequency $\left( \pm\right.$ SEM) of YFP $^{+}$microglia $\left(C^{\prime} 45^{+}\right.$Ly6C Ly6G $^{-}$F $\left.4 / 80^{+}\right)$and monocytes $\left(C D 45^{+}\right.$Ly6C $^{\text {hi } C D 11 b^{+} \text {Ly6G }}{ }^{-}$). (Pooled data of 2 individual experiments, $\left.n=3-5\right)$. (i) Quantitative $q P C R$ analysis of $/ 11$, Tnfa and Cxc/10 mRNA expression ( \pm SEM) of microglia (CD45 lo/int $\mathrm{Gr} 1^{-} \mathrm{F} 4 / 80^{+}$) sorted from LPS treated or untreated WT mice, and of tamoxifen treated Sall ${ }^{\mathrm{CreER}} \operatorname{Tgfbr}^{\mathrm{fl} / \mathrm{fl}}$ and $\mathrm{Tgfbr} 2^{\mathrm{fl} / \mathrm{fl}}$ mice on d0 and d6 after tamoxifen treatment. Data were normalized to Pol2 expression. ( $n=2$ replicates, 3 - 4 mice were pooled for each sample).

\subsection{Role of Sall1 and Tgfbr2 during microglia development}

As discussed in the introduction, microglia derive from primitive yolk sac progenitors that invade the developing CNS around E9 ${ }^{46}$. To assess whether these early progenitors already express Sall1 we set up timed matings of Sall $1^{\mathrm{GFP} /+}$ reporter mice and analyzed the CNS of the offspring at different embryonic and postnatal time points. We did not observe expression of Sall1 in yolk sac precursors or early embryonic microglia in the CNS at E11.5 (Figure 18a,b). We observed a the down-regulation of CD45 and F4/80 as well as the up-regulation of $\mathrm{CD} 11 \mathrm{~b}$ in microglia as they mature (Figure 18c). Sall1 is expressed in the majority of these cells starting at E14.5 (Figure 18a-c), when microglia also obtain their typical ramified morphology ${ }^{45}$ Sall 1 expression further increases so that all mature microglia $\left(C D 45^{10} \mathrm{~F} 4 / 80^{10} \mathrm{CD} 11 \mathrm{~b}^{\mathrm{hi}}\right)$ express Sall1 while immature precursors $\left(C D 45^{\text {hi }} F 4 / 80^{\text {hi }} C D 11 b^{\text {lo }}\right)$ do not express this signature gene (Figure 18a,b). Of note, $C D 11 \mathrm{c}$ expression is inversely regulated to $\mathrm{CD} 11 \mathrm{~b}$ and down-regulated with the maturation of microglia (data not shown). We set up timed matings of Sall $1^{\mathrm{GFP} /+}$ mice, to analyze homozygous Sall1 ${ }^{\text {GFP/GFP }}$ mice just before birth at E18.5. Due to the knock-in of GFP into the endogenous Sall1 locus, these mice have no functional Sall1 expression and would die within the first $24 \mathrm{~h}$ after birth ${ }^{86}$. Interestingly, despite the early expression of Sall1 in microglia it does not seem to be essential for their development, as we did not observe differences in microglia numbers between homozygous Sall $1^{\text {GFP/GFP }}$ mice and control Sall $1^{\mathrm{GFP} /+}$ or Sall $1^{\text {+/+ }}$ mice (Figure 18d). Similar results have also recently been described for Sall1deficient microglia in the retina, which develop in normal numbers but show a more 
activated morphology ${ }^{84}$. Further studies will be required to delineate the impact of Sall1 deficiency in microglia on their morphology, tissue distribution and function as well as the related effects on CNS development.

Based on our findings that TGF- $\beta$ R signaling in microglia is not crucial for their survival but is essential to keep them in a non-reactive state, we wanted to assess the importance of TGF- $\beta R$ signaling during microglia development. To address this, we set up timed matings of $C \times 3 c r 1^{\mathrm{CreER}} T g f b r 2^{\mathrm{fl} / f \mathrm{l}}$ as well as Sall1 ${ }^{\mathrm{CreER}} \mathrm{Tgfbr} 2^{\mathrm{fl} / \mathrm{fl}}$ mice and treated the pregnant mothers with Tamoxifen (5mg o.g.) at E9.5 or E14.5 respectively. Using this approach we were able to delete $T g f b r 2$ in early $\mathrm{CX}_{3} \mathrm{CR} 1^{+}$ yolk sac precursors and microglia ${ }^{46}$ or only in Sall $1^{+}$microglia. In contrast to recent reports ${ }^{50}$, we did not detect a decrease in overall microglia numbers between cre $^{+}$ animals and controls in either model. However we observed an arrest of microglial development in an immature state indicated by inversed ratios of $F 4 / 80^{\text {hi }}$ to $F 4 / 80^{\text {lo }}$ cells (Figure 18e,f). This change was more pronounced in Sall $1^{\mathrm{CreER}} \mathrm{Tgfbr}^{\mathrm{fl} / \mathrm{fl}}$ mice. Given the limited data on this topic so far, we cannot rule out a contribution of fetal liver derived monocytes to the population of $F 4 / 80^{\text {hi }}$ cells observed in conditional TGF- $\beta$ R deleted mice.

Taken together these results show that Sall1 is expressed early during development in CNS-resident microglia but is not essential for their survival. Likewise, TGF- $\beta$ R signaling is not essential for the survival of developing microglia however it is required for a proper maturation/differentiation of these cells. Further studies need to be performed to assess the different aspects of microglia development in more detail and follow manipulated microglia into adult mice. 
a

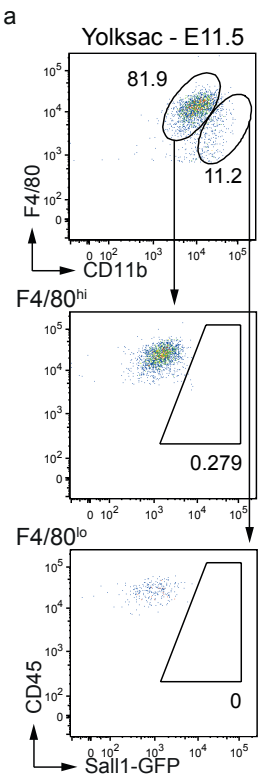

d
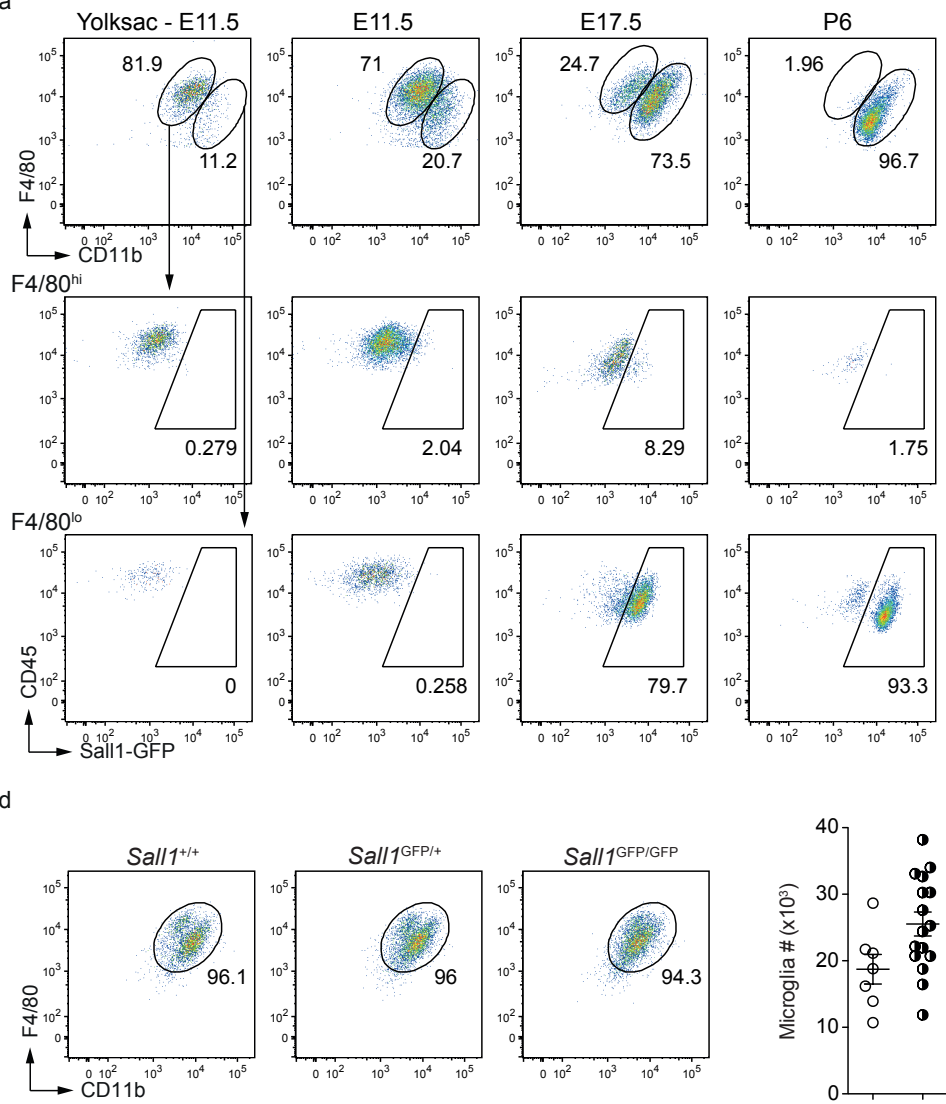

b

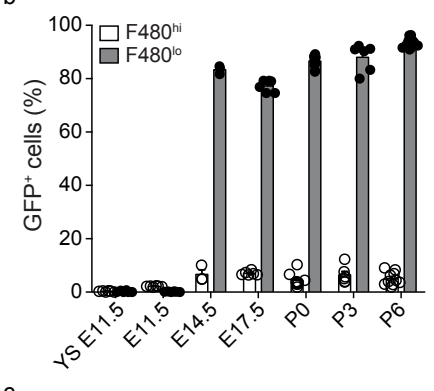

C
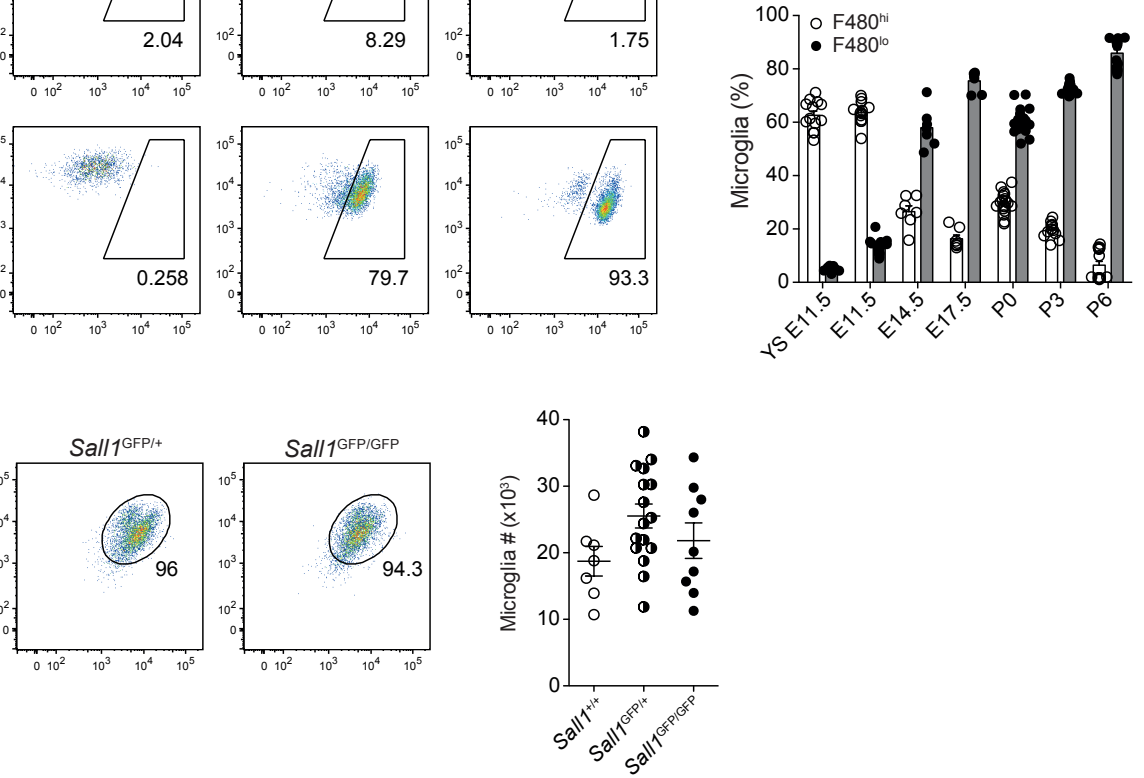

$$
\text { e }
$$

Tamoxifen at E9.5

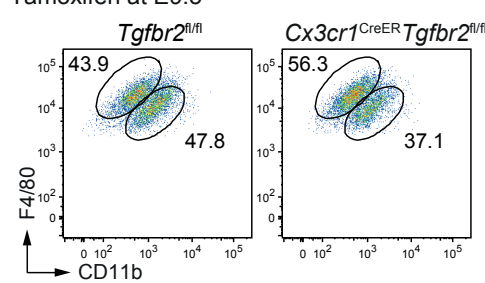

Tamoxifen at E14.5
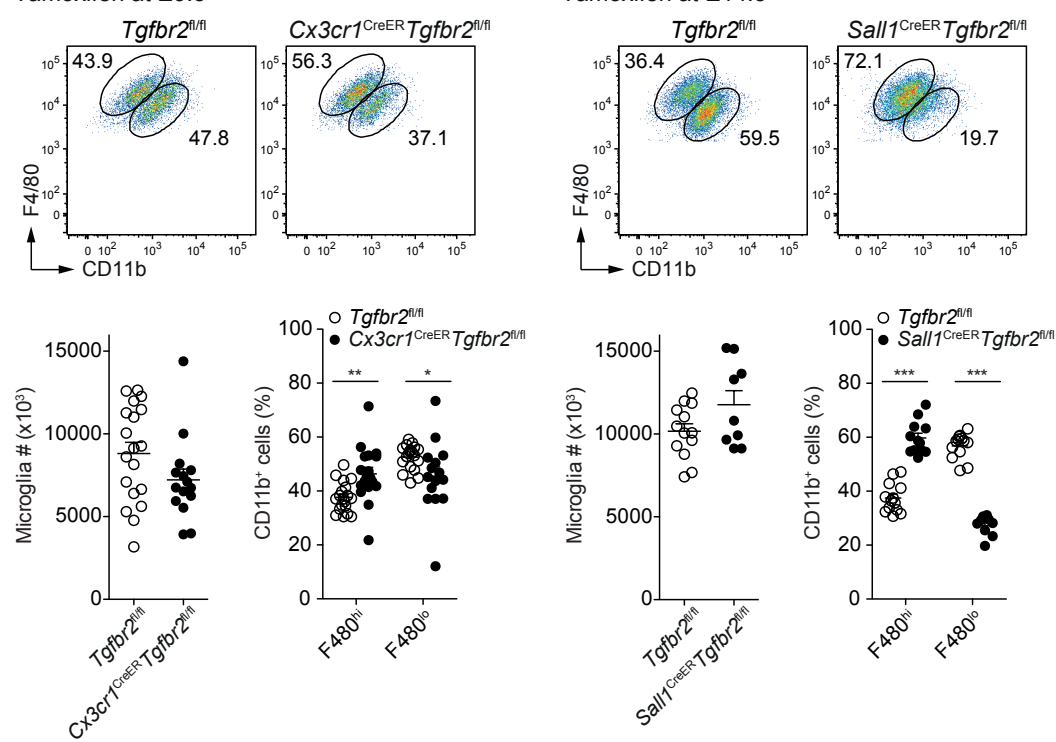

Figure 18 | Expression of Sall1 and Tgfbr2 during microglia development. (a) Representative flow cytometry plots show frequency of $\mathrm{GFP}^{+}$microglia $\left(\mathrm{F} 4 / 80^{\mathrm{hi}}\right.$ and $\mathrm{F} 4 / 80^{\mathrm{lo}}$, pregated on $\mathrm{CD} 45^{+}$Ly6C $\left.\mathrm{CH}^{-} \mathrm{GG}^{-}\right)$in the yolk sac (YS) and CNS of Sall ${ }^{\mathrm{GFP} / \mathrm{H}}$ mice at embryonic days E11.5 and E17.5 and at postnatal day P6. (Pooled data of 2 independent experiments, $n=3-10$. (b-c) Scatter plots show frequencies ( \pm SEM) of GFP ${ }^{+}$microglia (b) and of $F 4 / 80^{\mathrm{hi}}$ and $\mathrm{F} 4 / 80^{\mathrm{lo}}$ populations (c) during different developmental time points in $\mathrm{YS}$ and CNS of Sall $1^{\mathrm{GFP} / \mathrm{t}}$ mice. (Pooled data of 2 independent experiments, $n=7-19$ ). (d) Representative flow cytometry plots and scatter plots display frequencies and numbers ( \pm SEM) of microglia (pregated on CD $45^{+}$Ly6C $^{-}$Ly6G $^{-}$) in 
Sall $1^{+/+}$, Sall $1^{\text {GFP/+ }}$ and Sall $1^{\text {GFP/GFP }}$ mice at embryonic day E18.5. (Pooled data of 3 independent experiments, $\mathrm{n}=7-16)$. (e-f) Representative flow cytometry plots and scatter plots show frequencies and numbers $( \pm$ SEM) of microglia (pregated on CD45 ${ }^{+}$Ly6C $\mathrm{Ly}^{-} 6 \mathrm{G}^{-}$) at embryonic day E18.5 in Cx3cr1 ${ }^{\mathrm{CreER}} \mathrm{Tgfbr} 2^{\mathrm{fl} / \mathrm{fl}}$ and $\mathrm{Tgfbr} 2^{\mathrm{f} / / \mathrm{fl}}$ mice treated with a single dose of Tamoxifen (5mg o.g.) at E 9.5 (e) or in Sall ${ }^{\mathrm{CreER}}{ }^{\mathrm{Tgfbr} 2^{\mathrm{fl} / \mathrm{fl}}}$ and $T g f b r 2^{\mathrm{fl} / \mathrm{fl}}$ mice treated similarly at E14.5 (f). (Pooled data of 2 independent experiments, $n=9-19$ ).

\subsection{Sall1 controls the transcriptional signature of microglia}

Given the high expression of Sall1 in microglia, we sought to identify its function in vivo. The Sall $1^{\text {CreER }}$ allele has been targeted into the Sall1 locus, therefore Sall $1^{\text {CreER/fl }}$ mice (heterozygous for both alleles) allow inducible ablation of Sall 1 expression in microglia after tamoxifen treatment. We performed RNA-sequencing (RNA-seq) to examine gene expression profiles of microglia sorted from tamoxifen treated adult Sall1 $1^{\mathrm{CreER} / \mathrm{fl}}$ mice and Sall ${ }^{\text {fl/fl }}$ control littermates (Figure 19a,b). Microglia were obtained with $>98 \%$ purity and we did not observe differences in the numbers of microglia between Sall $1^{\mathrm{CreER} / \mathrm{fl}}$ and Sall1/1/fl mice (Figure 19b, data not shown). The absence of Sall1 was confirmed in Sall1 ${ }^{\text {CreER/fl }}$ microglia (Figure 19c). Hierarchical clustering of the differentially expressed genes showed a clear segregation between control and Sall1-deficient microglia (Figure 19d). Transcriptional changes assessed by RNA-seq revealed that from a total of 12,673 expressed genes, 847 genes were significantly up-regulated and 608 genes were down-regulated in microglia lacking Sall1 (Figure 19e,f). Among the fifty most significantly altered expression features, genes associated with inflammation and activation were up-regulated in microglia deficient in Sall1 (Figure 19e,g). 
In the past few years it has become clear that not only the ontogeny varies between different macrophage populations but also their gene expression signatures, which can be imprinted by the local tissue microenvironment ${ }^{30,31}$. By comparing differentially regulated genes of control microglia vs. Sall1-deficient microglia, we found that many of the previously described microglia signature genes (as shown in ${ }^{32,106}$ ) such as P2ry12, Serpinf1, or S/c2a5, were downregulated upon Sall1-deletion (Figure 19h). In addition, genes associated with the identity of other tissue macrophages were induced in microglia upon deletion of Sall1 ${ }^{32}$. Among those were Msr1 and Stard13, usually specifically expressed in peritoneal macrophages, Cyp4f18, Itgal and Cd69, typically high in alveolar macrophages and Spic, Vcam1, Igf1 and Ahr normally found at high levels in splenic red pulp macrophages (Figure 19h).

Similar changes in gene expression profiles were found in Sall1-deficient microglia isolated from tamoxifen-treated $\mathrm{C} \times 3 \mathrm{Cr} 1^{\mathrm{CreER}}$ Sall $1^{\mathrm{fl} / \mathrm{fl}}$ mice. In these mice, deletion of Sall1 is targeted to $\mathrm{CX}_{3} \mathrm{CR} 1^{+}$myeloid cells including microglia and CNS-associated macrophages but not to any other CNS-resident cells as previously shown (Figure 19d, Figure 13f) ${ }^{19,107}$. This experiment clearly demonstrates that Sall1 acts in a microglia-intrinsic manner and is not the result of collateral Sall1 loss outside the microglia compartment. Overall these data demonstrate a pronounced change in microglia phenotype due to the deletion of a single transcription factor. 
b
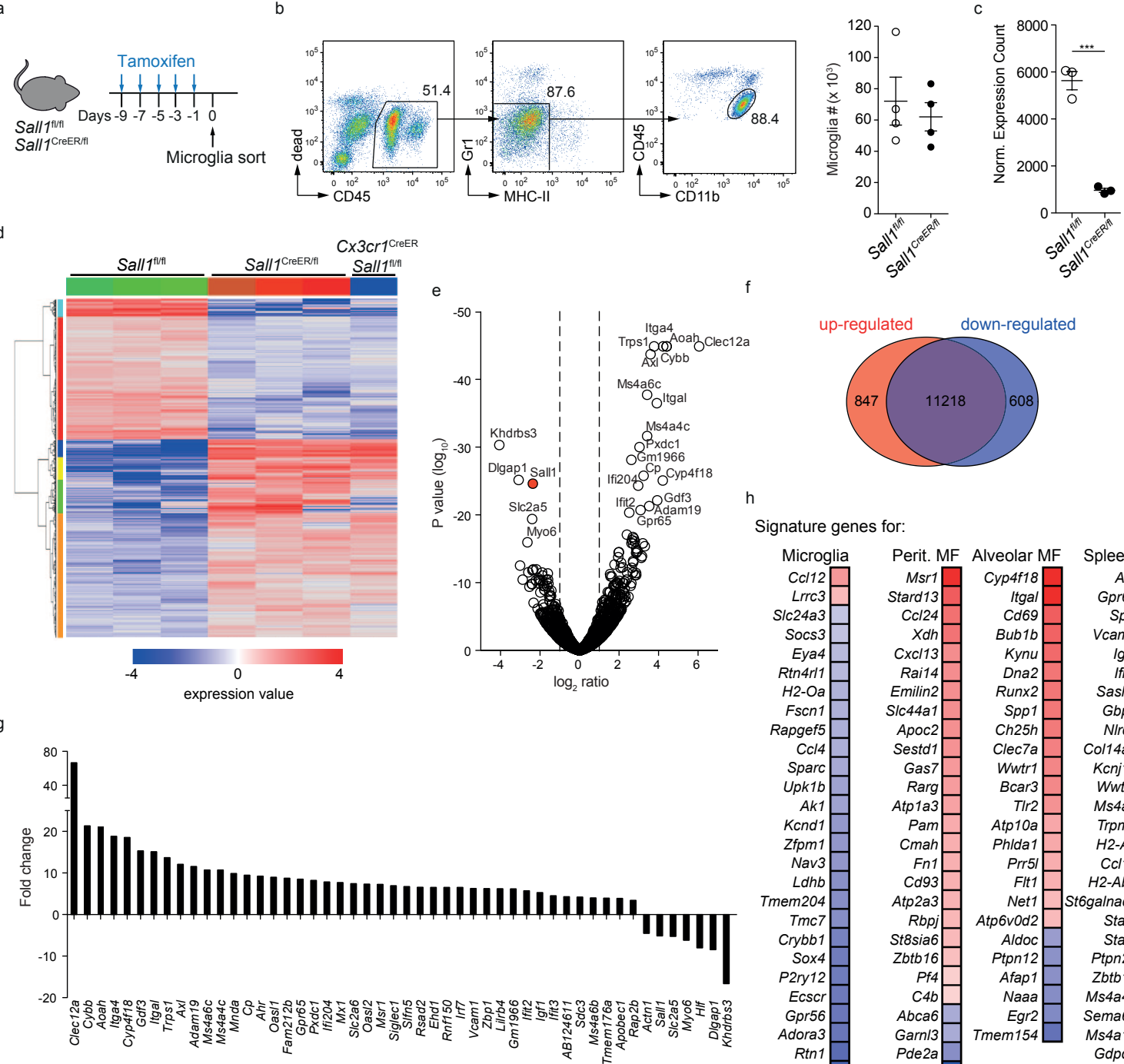

\section{.}

up-regulated down-regulated

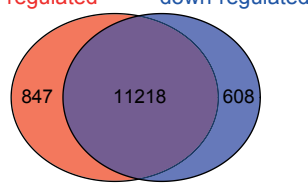

h

Signature genes for:

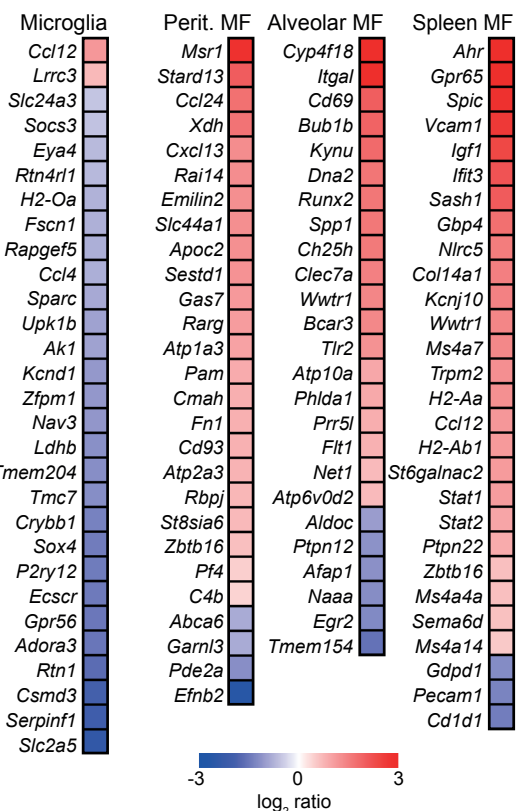

Figure 19 | Gene expression profile of Sall1-deficient microglia. (a) Scheme of the experimental setup. Sall $1^{\text {CreER/fl }}$ and Sall $1^{\text {fl/fl }}$ mice were treated 5 times with tamoxifen $\left(2.5 \mathrm{mg}\right.$ i.p.) every $2^{\text {nd }}$ day and microglia were sorted one day after the last treatment. (b) Gating strategy and cell numbers ( \pm SEM) of sorted microglia used for NGS. $(n=4)$. (c-h) Gene expression data obtained from NGS (3 replicates of Sall $1^{\text {CreER/fl }}$ and Sall/ $1^{\mathrm{fl} / \mathrm{fl}}$ mice, one sample of $\mathrm{Cx} 3 \mathrm{cr} 1^{\mathrm{CreER}}$ Sall $1^{\mathrm{fl} / \mathrm{fl}}$ mice , 3-5 mice pooled per sample). (c) Normalized expression count ( \pm SEM) of Sall1 expression in microglia. Unpaired $t$-test, ${ }^{* * *} p<0.001$. (d) Heat map and cluster analysis of differentially expressed genes. 471 genes were significantly altered to a minimum significance threshold of $p<$ 0.001. (e) Volcano plot showing $\log _{2}$ ratios vs. p values $\left(\log _{10}\right)$ of all 12,673 detected genes. Genes with highest significance values are annotated. (f) Venn diagram of differentially expressed genes. (g) Bar graph displays the fold change of the 50 most significantly altered genes of microglia derived from tamoxifen-treated Sall $1^{\text {CreER/fl }}$ compared to Sall $1^{\mathrm{fl} / \mathrm{fl}}$ mice. (h) Heat maps display $\log _{2}$ ratios of macrophage signature genes regulated by Sall1 clustered according to their tissue residence. 


\subsection{Sall1 regulates the microglia phenotype and function during tissue homeostasis and disease}

Pathway analysis performed by MetaCore ${ }^{\mathrm{TM}}$ demonstrated that genes involved in immune system (IS) responses including cytokines/chemokines, cell cycle, phagocytosis and neurogenesis were most differently regulated in microglia lacking Sall1 (Figure 20a). For example, the pro-inflammatory oxidase Nox2 (Cybb), which is implicated in microglia-mediated neurotoxicity ${ }^{106,108}$, was highly expressed by Sall1-deficient microglia but only at low levels in control microglia. The expression of interferon response and regulatory genes such as Ifit3, Ifit2, Ifitm3, Mx1 and Irf7, which are usually expressed under inflammatory conditions, were greatly enhanced in Sall1-deficient microglia ${ }^{109,110}$. The aryl hydrocarbon receptor (Ahr), usually not present on microglia isolated from healthy brain, was expressed in microglia lacking Sall1. Ahr is a transcriptional regulator critical for innate immunity by promoting macrophage survival and ROS production among other functions 111,112. $A x l$ is part of a phagocytic receptor and expressed at low levels in the healthy CNS but it is up-regulated in microglia in inflammation ${ }^{113}$. Microglia lacking Sall1 also show significantly elevated expression levels of Axl. Along those lines, we assessed the phagocytic capacity of ex vivo isolated microglia from Sall $1^{\text {CreER/fl }}$ mice in comparison to controls using $\mathrm{pH}$-sensitive pHrodo bioparticles. Comparing the frequency of pHrodo $^{+}$particles in microglia after $4 \mathrm{~h}$ of culture we observed a significant increase in phagocytic activity in Sall1-deficient microglia (Figure 20b,c).

Additionally, we wanted to address whether Sall1 deletion in microglia would impact the outcome of neuro-inflammatory disorders. To assess this we immunized Sall $1^{\text {CreER/fl }}$ and Sall $1^{\mathrm{fl} / \mathrm{fl}}$ mice s.c. with $\mathrm{MOG}_{35-55} / \mathrm{CFA}$ as described before to induce EAE. Monitoring the animals until peak disease, we did not observe vast differences in the clinical scores (Figure 20d). Similarly, the day of disease onset and the maximal clinical score did not vary between experimental and control group (Figure 20e). When we analyzed the CNS of immunized animals at peak disease, we observed a significant increase in infiltrating MCs while the number of other myeloid cells present at that time point remained similar (Figure 20f,g). This indicates that the cellular composition in the CNS during neuro-inflammation is altered upon Sall1 deletion in microglia. The effects of this compositional change 
on the clinical outcome and the recovery phase remain to be monitored in more detail. Altogether these data propose that Sall1 is required for microglia to maintain their physiological expression phenotype to fulfill their homeostatic functions in the CNS and protect the sensitive surrounding tissue. Further studies will be necessary to characterize the impact of the loss of Sall1 in microglia in more detail.

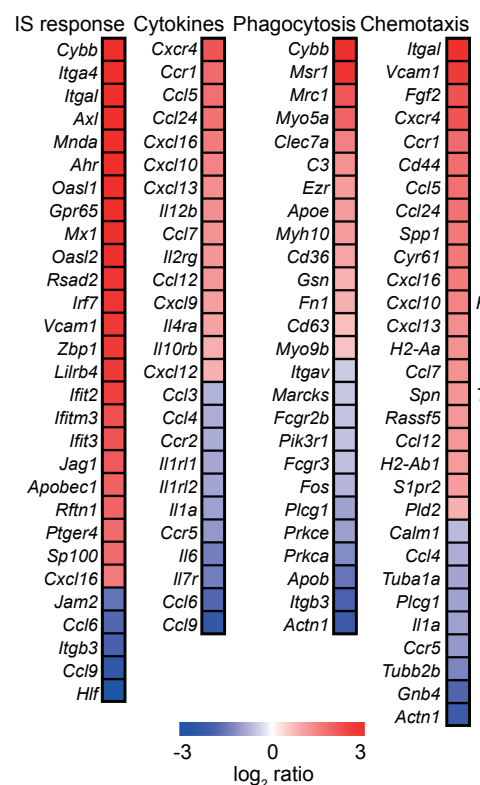

d

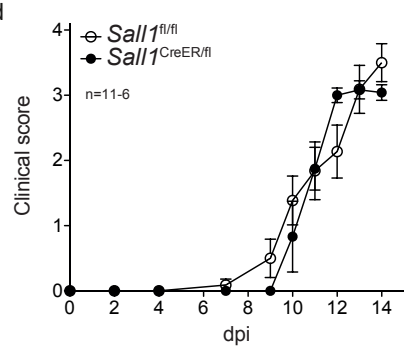

e

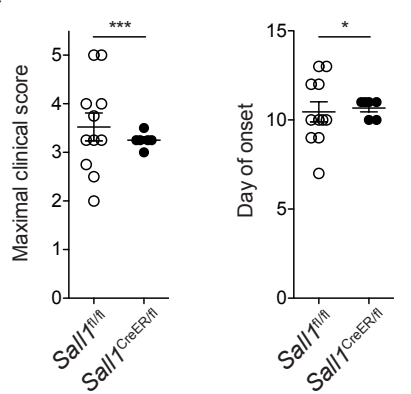

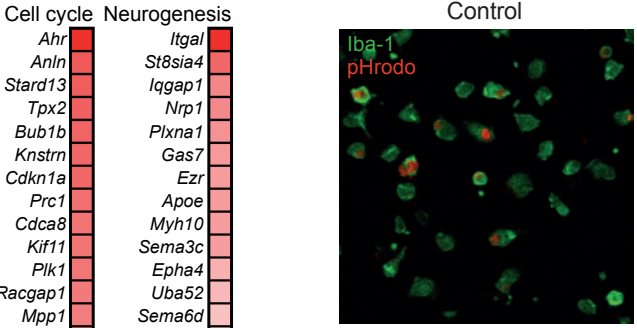

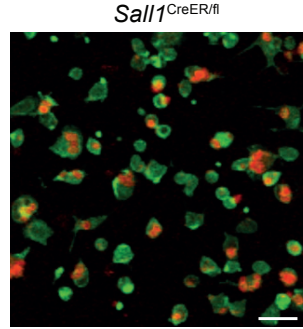

c
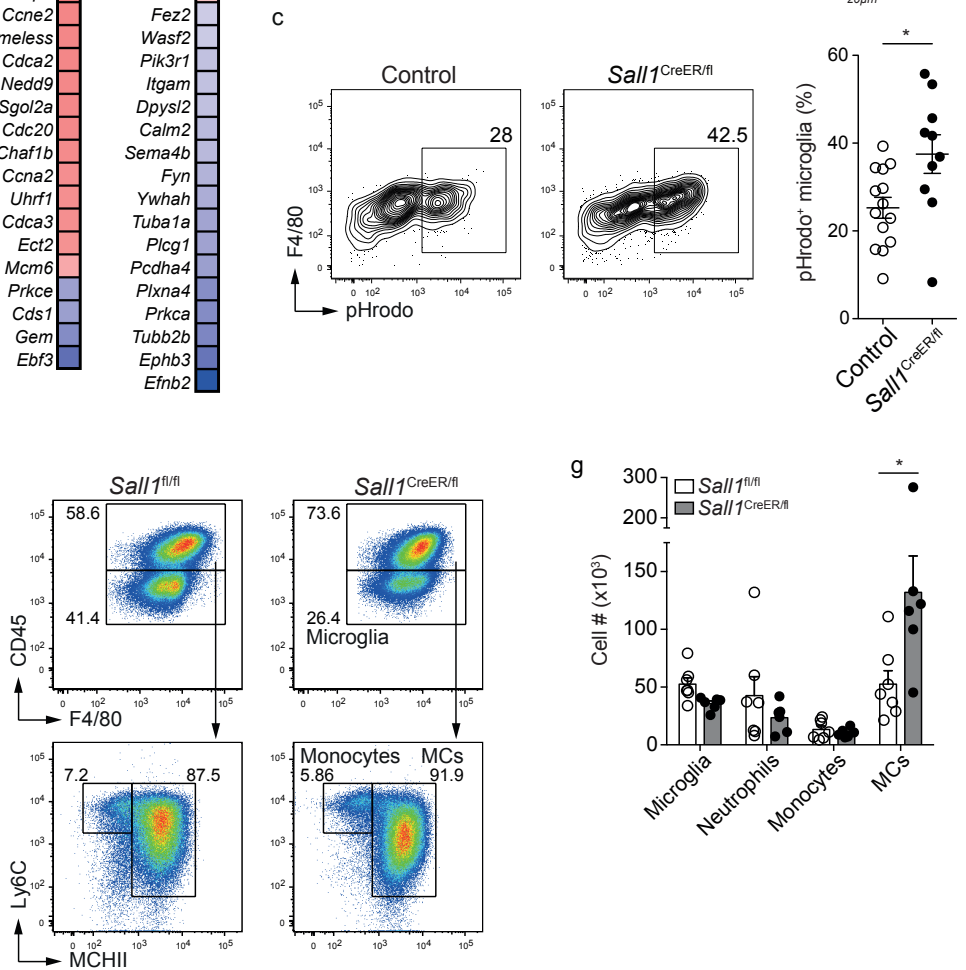

Figure 20 | Sall1-deficient microglia show altered functional properties. (a) Gene expression analysis of microglia derived from tamoxifen treated Sall1 ${ }^{\mathrm{CreER} / \mathrm{fl}}$ and Sall $1^{\mathrm{fl} / \mathrm{fl}}$ mice as described in Figure 19. Heat maps display $\log _{2}$ ratios of Sall1-regulated genes clustered to their indicated GO-pathways. IS: Immune system. (bc) Analysis of phagocytic activity of microglia ex-vivo isolated from tamoxifen treated Sall/ $1^{\text {CreER/fl }}$ and Sall $1^{\text {fl/f }}$ mice. (b) Representative IHC for Iba-1 (green) and pHrodo (red) display phagocytically active microglia. 
(c) Representative flow cytometry plots and scatter plots show frequencies $( \pm \mathrm{SEM})$ of $\mathrm{pHrodo}^{+}$microglia (pregated on $\mathrm{CD} 45^{+} \mathrm{CD} 11 \mathrm{~b}^{+} \mathrm{Gr} 1^{-}$). (Pooled data of 3 independent experiments, $\left.\mathrm{n}=10-14\right)$. (d-g) Tamoxifen treated Sall ${ }^{\mathrm{CreER} / \mathrm{fl}}$ and Sall ${ }^{\mathrm{fl} / \mathrm{fl}}$ mice were analyzed at peak disease of $\mathrm{MOG}_{35-55} / \mathrm{CFA}$ induced EAE. $(n=6-11)$ (d) Graph displays clinical score ( \pm SEM) over days post injection (dpi). (e) Scatter plots show maximal clinical score and day of onset of clinical EAE disease symptoms ( \pm SEM). (f) Representative flow cytometry plots show frequencies of microglia, monocytes and MCs (pregated on CD45 $5^{+}$D $11 \mathrm{~b}^{+} \mathrm{Ly} 6 \mathrm{G}^{-}$). (g) Bar graphs display frequencies $\left( \pm \mathrm{SEM}\right.$ ) of neutrophils (gated on $\mathrm{CD} 45^{+} \mathrm{CD} 11 \mathrm{~b}^{+} \mathrm{Ly} 6 \mathrm{C}^{+}$) and microglia, monocytes and MCs (gated as shown in $\mathrm{f}$ ) in the CNS.

In line with the RNA-seq data showing that Sall1 maintains the transcriptional signature specific to microglia, we also observed significant morphological alterations, like shorter, thicker processes and bigger cell soma, by using 3D reconstruction of individual microglia cells (Figure 21a, b). The morphological change coincided with an increase in microglial cell numbers in the hippocampus (Figure 21c).

Among others adult hippocampal neurogenesis occurs in the subgranular zone (SGZ) of the dentate gyrus, where GFAP-expressing radial-glia-like stem cells give rise to doublecortin $(\mathrm{DCX})^{+}$neuroblasts, which then differentiate into new granule cell neurons ${ }^{114}$. Multiple factors including pathological conditions can influence adult neurogenesis as reflected by altered proliferation, differentiation and survival of precursor cells. We found the number of GFAP-expressing radial-glia-like stem cells and the number of proliferating $\left(\mathrm{DCX}^{+} \mathrm{Ki}^{+} \mathrm{T}^{+}\right)$neuroblasts unchanged in the dentate gyrus of tamoxifen treated Sall $1^{\mathrm{CreER} / \mathrm{fl}}$ mice compared to control mice. However differentiating $\left(\mathrm{DCX}^{+} \mathrm{Ki}^{-} 7^{-}\right)$neuroblasts were significantly decreased in Sall $1^{\mathrm{CreER} / \mathrm{fl}}$ mice (Figure 21d-f). Similar results were obtained in tamoxifen treated $C \times 3 c r 1^{\text {CreER }}$ Sall $1^{\text {fl/fl }}$ mice (Figure $21 \mathrm{~g}$ ) again demonstrating that the phenotype is driven by microglia-intrinsic processes.

Of note, the few Sall1 expressing CD45 cells in the adult liver and kidney (Figure 11c) are also targeted using Sall $1^{\mathrm{CreER} / \mathrm{fl}}$ mice. After tamoxifen treatment, Sall1 deletion in these cells did not result in overt tissue inflammation as assayed by multiplex immunoassay in the serum, spleen, liver and kidney. IL-1, IL-6, TNF- $\alpha$ and IL-10 were detected at low levels but no major differences were found between tamoxifen treated Sall $1^{\mathrm{CreER} / \mathrm{fl}}$ and Sall $1^{\mathrm{fl} / \mathrm{fl}}$ animals (Figure 21h). GM-Csf, IFN-Y, IL2 and IL-12 were below detection limit (data not shown). In addition, after long-term 
tamoxifen treatment, Sall $1^{\text {CreER/fl }}$ mice remained viable and healthy and no apparent clinical phenotype was observed (data not shown).

If the cell-intrinsic loss of Sall 1 leads to microglia activation, we questioned whether extrinsic activation triggers would in turn regulate Sall1. We injected WT mice with LPS i.p. and sorted microglia $24 \mathrm{~h}$ later. Microglia isolated from LPS-challenged mice down-regulated Sall1 compared to untreated control mice (Figure 21i). As demonstrated earlier, loss of TGF- $\beta$ R signaling likewise results in aberrant reactive microglia, which interestingly also showed reduced levels of Sall1 (Figure 21i).

Taken together, these data support that under physiological conditions, the function of Sall1 is to repress 'activatory' genes in microglia and conversely, Sall1 down-regulation / deletion allows microglia activation. In turn this demonstrates, that Sall1-deficiency in microglia results in disturbed CNS homeostasis as demonstrated by altered neurogenesis. 

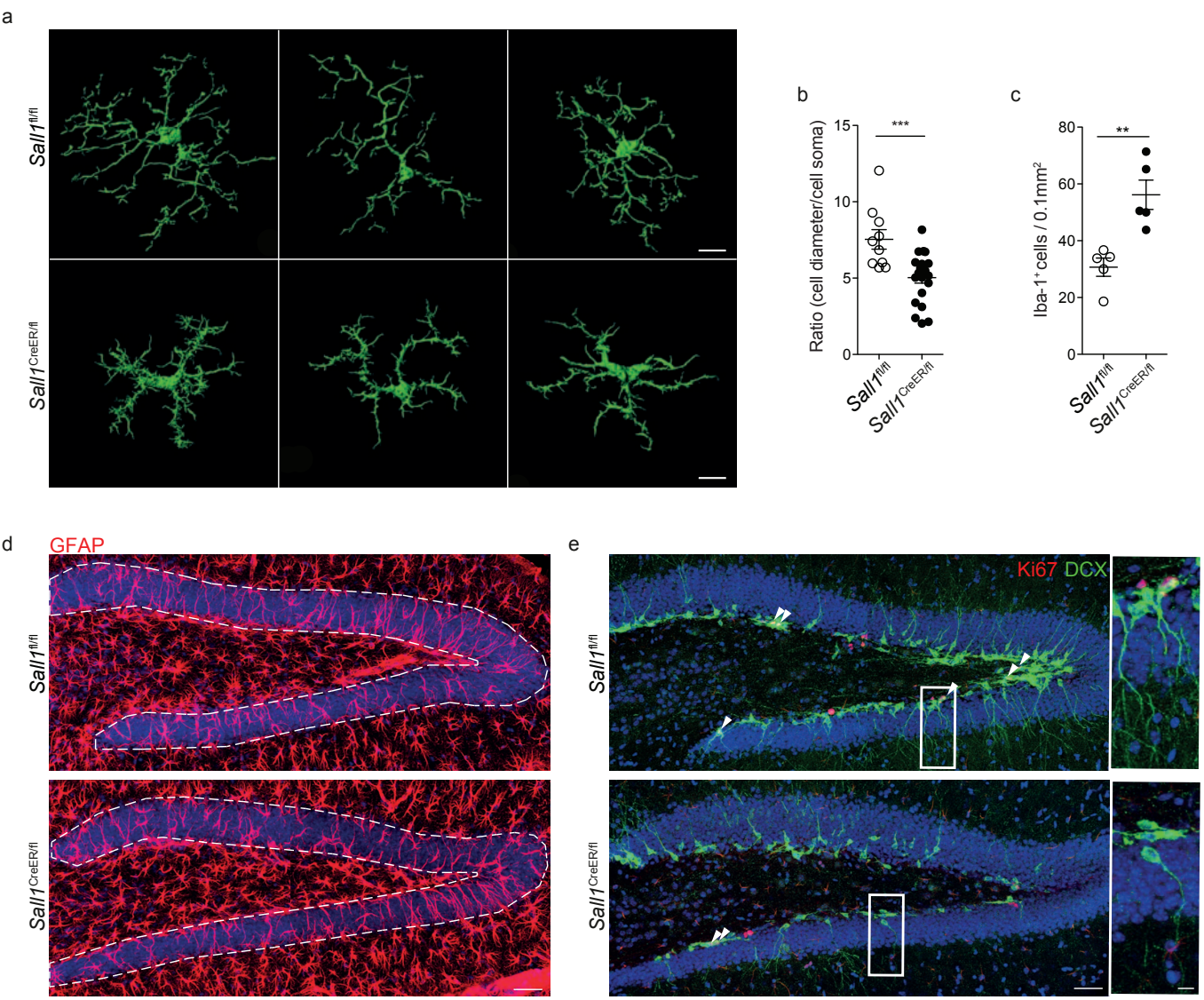

f

g
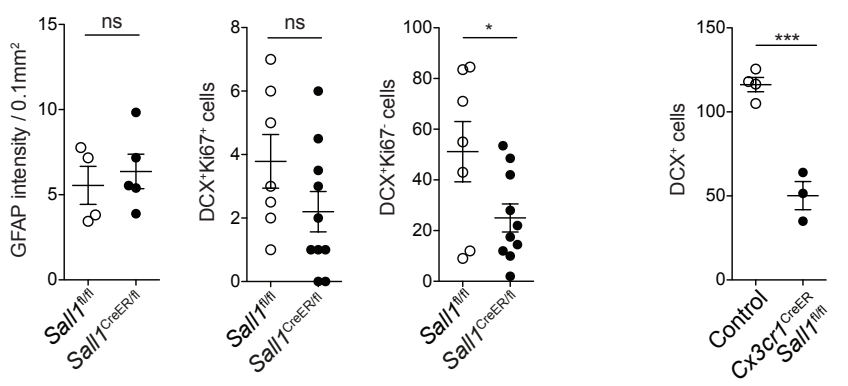

h
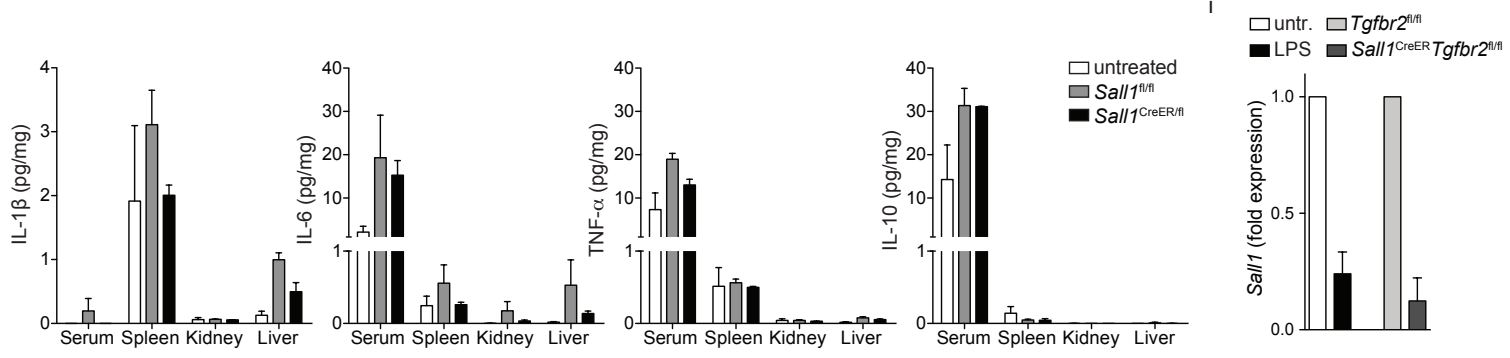

Figure 21 | Sall1 deletion in microglia leads to changes in adult neurogenesis. (a-f) Sall $1^{\mathrm{CreER} / \mathrm{fl}}$ and Sall $1^{\mathrm{fl} / f l}$ mice were treated with tamoxifen food for at least 5 weeks. (a) $3 \mathrm{D}$ reconstruction of Iba- $1^{+}$(green) microglia in tamoxifen-treated Sall $1^{\mathrm{CreER} / \mathrm{fl}}$ and Sall $1^{\mathrm{fl} / \mathrm{fl}}$ mice. Representative images in cortex and hippocampus. (Scale bar: $10 \mu \mathrm{m}$ ). (b) Quantification ( \pm SEM) of the ratio of the microglial cell body diameter at its widest expansion including its processes over the cell soma diameter. ( $n=10-21$ microglial cells in hippocampus, cortex, cerebellum. Pooled data of 3 mice). (c) Graph shows cell counts of lba-1+ cells in hippocampal CNS sections of tamoxifen treated Sall $1^{\text {CreER/fl }}$ mice and Sall $1^{\mathrm{flfl} l}$ littermates. (2-3 sections counted, pooled data of 2 mice). 


\section{Results}

(d-e) IHC of CNS sections (hippocampus) of tamoxifen treated Sall1 ${ }^{\text {CreER/fl }}$ mice and Sall $1^{\mathrm{fl} / \mathrm{fl}}$ littermates for DAPI (blue), GFAP (red, astrocytes and neuronal progenitor cells) (d) or DAPI (blue), doublecortin (DCX, green, neuroblasts) and Ki67 (red, proliferating cells) (e). Arrowheads in e) indicate proliferating neuroblasts. Small images display the magnification of the inset in the overview picture. (Scale bar: $50 \mu \mathrm{m}$, inset: $20 \mu \mathrm{m}$ ). (f) Graphs ( \pm SEM) display GFAP staining intensities in the granule cell layer (indicated by the dotted line in d) as well as cell counts of proliferating $\left(\mathrm{DCX}^{+} \mathrm{Ki} 67^{+}\right)$and differentiating $\left(\mathrm{DCX}^{+} \mathrm{Ki67}\right)$ neuroblasts (as in e). $(\mathrm{n}=4-10$ mice). (g) Graph ( \pm SEM) displays cell counts of $D C X^{+}$neuroblasts in hippocampal brain sections of tamoxifen treated $C \times 3 c r 1^{\mathrm{CreER}}$ Sall ${ }^{\mathrm{fl} / \mathrm{fl}}$ and Cre ${ }^{-}$control littermates $(\mathrm{n}=3-4)$. (h) Graphs ( \pm SEM) display levels of IL-1, IL-6, TNF- $\alpha$ and IL-10 (pg/mg) in serum and whole tissue lysates of spleen, kidney and liver of untreated control mice and tamoxifen treated Sall $1^{\text {CreER/fl }}$ and Sall $1^{\mathrm{fl} / \mathrm{fl}}$ mice at $\mathrm{d} 6$ after treatment start assessed by multiplex immunoassay. ( $n=3$ for Sall $1^{\text {CreER/fl }}$ and Sall $1^{\text {fl/fl }}$ mice, $n=2$ for untreated control mice). (i) Quantitative $q P C R$ analysis of Sall1 mRNA expression of microglia $\left(C D 45^{\text {lo-int }} \mathrm{Gr} 1^{-} \mathrm{CD} 11 \mathrm{~b}^{+}\right)$sorted from LPS treated or untreated WT mice, and of tamoxifen treated Sall $1^{\mathrm{CreER}} \mathrm{Tgfbr}^{\mathrm{fl} / \mathrm{fl}}$ and $\mathrm{Tgfbr}^{\mathrm{fl} / \mathrm{fl}}$ mice on d6 after tamoxifen treatment (as in Fig. 3d). Data were normalized to Pol2 expression. ( $n=2$, 3-4 mice were pooled for each sample). Unpaired $t$ test, ${ }^{*} \mathrm{p}<0.05,{ }^{* *} \mathrm{p}<0.01,{ }^{* *} \mathrm{p}<0.001(\mathrm{~b}, \mathrm{c}, \mathrm{f}, \mathrm{g})$. 


\section{Discussion}

\subsection{Identification of Sall1 as a transcription factor specific to microglia}

Over the past decade, it has become clear that most tissue macrophages have embryonic origins, and are self-maintained throughout adulthood in the absence of inflammatory incidents, rather than being constantly replenished by circulating monocytes ${ }^{115}$. Initially, macrophages were considered to perform primarily immune-related functions. But a new picture is emerging, in which control and regulation of tissue homeostasis are carried out by local macrophage populations. Advances in cell fate mapping and deep transcriptional profiling illustrate a highly specialized expression signature within tissue macrophages, depending on the tissue environment in which they reside ${ }^{30-32}$. Despite the intensive research our understanding of microglia biology is still rather limited due to technical limitations and adverse targeting effects of previous models. The generation of the $C \times 3 c r 1^{\text {CreER }}$ strains, in which successful targeting in microglia can be achieved, have advanced the microglia field significantly ${ }^{19,59}$. However other myeloid cells expressing the fractalkine receptor $\left(\mathrm{CX}_{3} \mathrm{CR} 1\right)$ including kidney and intestinal macrophages are also targeted. Furthermore, $\mathrm{C} \times 3 \mathrm{Cr} 1$ is highly expressed by CNSassociated MF in the meninges, choroid plexus and perivascular space generating an important drawback of this model with regards to dissecting the individual contributions of CNS-resident microglia and CNS-associated MF in settings of CNS homeostasis and inflammation. Here we identified the transcriptional regulator Sall1 as virtually exclusive to microglia within the MPS and wider hematopoietic system as well as within the adult CNS. Interestingly, Langerhans cells, which are partly derived from yolk sac macrophages as well ${ }^{116}$, do not express Sall1. Similarly in the testes, which are also considered partially immune privileged like the CNS, macrophages are negative for Sall1. In addition, Sall1 expression was not detected in BM-derived microglia-like cells repopulating the empty microglia niche after depletion in a chimeric setting. This suggests that microglia identity and their characteristic expression of Sall1 are made up of a combination of ontogeny and imprinting by the local tissue environment. The specificity of Sall1 to bona fide microglia finally allows for microglia specific targeting in vivo via the Sall1 locus. 


\subsection{Targeting microglia in vivo using the Sall1 locus}

Using knockout mice it has been well established that Csf-1R signaling is crucial for the development of microglia ${ }^{46}$. Based on these finding and the data obtained from treatment with Csf-1R inhibitors it has also been claimed that Csf-1R signaling is essential for microglia survival and homeostasis under steady state conditions ${ }^{46,101}$. However this has never been conclusively demonstrated due to drawbacks in the respective models like impaired survival of Csf-1R knockout mice ${ }^{28}$ or targeting of other receptor tyrosine kinases like FLT3 or KIT by the use of Csf-1R inhibitors from Plexxikon ${ }^{51}$. Utilizing the tamoxifen-inducible Sall $1^{\text {CreER }}$ mouse strain, here we were able to show for the first time that Csf-1R signaling is indeed critical for the homeostasis and maintenance of adult microglia. By treating adult Sall1 ${ }^{\text {CreER }}$ Csf1 fl/fl mice with tamoxifen, we observed a pronounced reduction of microglia numbers in all brain areas compared to littermate controls. As described previously ${ }^{101}$, the empty microglia niche is repopulated within 7 days by the CNSintrinsic proliferation of microglia that in all likelihood escaped targeting.

In addition to Csf-1R, TGF- $\beta$ signaling has been claimed to be crucial for microglia development and possibly also homeostasis ${ }^{50}$. By specifically deleting TGF- $\beta R$ from microglia under steady state conditions we show that loss of TGF- $\beta R$ signaling results in the rapid transformation of surveying microglia towards reactive phagocytes while microglia numbers remain largely unchanged. The slight increase in microglia numbers observed at day 6 after tamoxifen treatment can be attributed to the increased proliferation of the activated TGF- $\beta R$-deficient microglia. Furthermore when we deleted TGF- $\beta R$ from microglia during embryonic development, we observed alterations in the maturation status of microglia, while the total cell number again remained unchanged. This suggests that TGF- $\beta R$ signaling is not critical for microglia survival but allows for proper maturation and a homeostatic activation profile. Further studies will be needed to dissect and define the role of TGF- $\beta R$ in microglia in greater detail. Along those lines, there might be a link between TGF- $\beta R$ signaling and Sall1 expression and/or function in microglia. For wing morphogenesis in Drosophila, it has been shown that expression of spalt and spalt-related gene are activated in response to the TGF- $\beta$ homologue decapentaplegic $(d p p){ }^{117}$. Our data show that similar to activated microglia of LPS treated mice, Sall1 expression is strongly reduced in microglia lacking TGF- $\beta R$. 
Taken together, these findings raise the question whether there is an interaction between Sall1 and Tgfb or downstream mediators of TGF- $\beta$ signaling on a genetic level. Further investigations will be needed to identify potential interactions and regulatory sites that influence Sall1 expression.

\subsection{The role of Sall1 in microglia}

It has been shown for kidney and limb organogenesis that Sall1 is required for the correct tissue patterning and is involved in the co-ordination of inducer signals between different tissue compartments ${ }^{85,86}$. Sall1 also seems to be involved in early steps of cortical neurogenesis by promoting proliferation of precursor cells over differentiation into intermediate progenitors ${ }^{91}$. These functions of Sall1 might be related to its interaction with genes implicated in embryogenesis and pluripotency such as $\beta$-Catenin/Wnt or Nanog respectively ${ }^{90}$. So far a short and rather abundant binding sequence has been described for Sall $1{ }^{118,119}$, which can be found in the promoter region of many potentially 'Sall1-regulated' genes. However the direct binding of Sall 1 to these genes and the functional consequences of such binding require further investigations.

In the case of murine microglia it is clear that Sall 1 is highly expressed yet its functions are largely unknown. We propose Sall1 to be a transcriptional master regulator of microglia identity by controlling their unique transcriptional profile and maintaining their non-reactive phenotype. Here we show that deletion of Sall1 in microglia results in their activation on a genomic as well as phenotypic level and leads to alterations in CNS homeostasis. Microglia have been shown to actively participate in adult neurogenesis by clearing excessively generated, apoptotic neurons ${ }^{62}$. Here we show that the phagocytic activity of Sall1-deficient microglia is increased compared to controls. And that microglia specific deletion of Sall1 results in dysregulated hippocampal neurogenesis indicated by decreased numbers of differentiating neuroblasts. It is tempting to speculate about a potential link between increased phagocytosis of microglia and reduced number of neuroblasts, however more detailed studies will be needed to investigate this. Furthermore it would be interesting to address whether the observed changes in neurogenesis due to loss of Sall1 in microglia result in impaired memory formation and impact on cognitive and behavioral function as it has been proposed for other models ${ }^{120}$. 
Even though Sall1 is not essential for microglia development, as Sall1 knockout mice display similar microglia numbers to controls, the functional impact of this deletion during embryogenesis remains to be shown. Since Sall1 knockout mice die within the first $24 \mathrm{~h}$ due to severe kidney dysgenesis or agenesis and given that neuronal precursors also depend on Sall1 during embryonic development, targeted deletion of Sall1 to individual CNS compartments will be required. Mouse strains like Vav1 ${ }^{\text {Cre }}$ or Nestin ${ }^{\text {Cre }}$ could allow for a specific deletion of Sall1 in microglia vs. neurons and glial precursors respectively and provide insights into the individual contributions of each compartment to embryonic neurogenesis.

\subsection{Microglial contributions to CNS damage and inflammation}

In addition to their homeostatic functions, microglia have also been implicated in neurodegenerative diseases, CNS injury and aging related CNS alterations. The exact contributions of microglia to these processes however remain a matter of debate and current concepts attribute them beneficial as well as detrimental functions. In the case of EAE as a model of autoimmune demyelination, we could show that deletion of Sall1 in microglia leads to an increased number of infiltrating monocytes-derived cells at peak disease, while disease onset and clinical score do not differ at that time point. It has been suggested previously that microglia mostly release mediators that promote the encephalitogenic potential of autoreactive $T$ cells, the infiltration and activation of inflammatory monocytes as well as the tissue destruction by ROS ${ }^{67-71}$. It would be interesting to see if previous activation of microglia by the deletion of Sall1 affects their cytokine production, phagocytic activity and inflammatory response and if that in turn leads to alterations in disease progression and/or recovery. This is not only true for tissue destructive actions such as in models of EAE or ALS but also for tissue repair for example by clearance of $A \beta$ plaques in $A D$ and of cellular debris after injury. Furthermore, targeted modulation of microglia via the Sall1 locus offers a new route of therapeutic interventions to reduce tissue injury and promote repair mechanisms. 


\subsection{Translation of the Sall1 specificity to human}

So far functional investigations and genetic profiling of different cell populations are mainly based on mouse models. First gene expression studies suggest that human microglia also express SALL1 ${ }^{121}$. To this day however there are no detailed datasets available comparing distinct human macrophage populations throughout different tissues obviating any claim of SALL1 specificity to human microglia similar to that of mice. One study providing gene expression profiling of human CNS cells with the focus on astrocytes suggested that other CNS resident cells might also express SALL ${ }^{121}$. However, so far this is the only data set of such kind and needs to be verified. We would therefore be reluctant to assign a specific gene expression to human microglia based on this data set only. In addition, transcription and translation are often not identical, due to post-transcriptional, translational, posttranslational and degradative modifications and protein data of human tissues is very sparse. Immunohistochemistry of human brain biopsies using a Sall1-specific antibody could address this question. To this day, based on the limited resources, the specificity of SALL1 expression in human myeloid cells and the CNS stroma remains to be shown. However it might very well be that there are species-specific differences in Sall1 expression.

Collectively, the ability to target microglia and microglia function through Sall1 allows for an unprecedented level of specificity. Moreover, the identification of Sall1 as a microglia fate regulator will better enable us to identify the relevant biological roles microglia play in maintaining proper CNS function. 
Discussion 


\section{Closing remarks}

Microglia-specific conditional gene-targeting has been a challenge for a long time and has hampered our progress in understanding the function of these CNSresident macrophages during development, health and disease. Here, by using Sall $1^{\text {CreER }}$ mice, we demonstrate microglia-specific targeting without targeting of other members of the MPS, wider hematopoietic system and adult CNS. We found Csf-1R signaling to be required for the survival of adult microglia. This has been suggested in the past but until now never conclusively been demonstrated. Likewise TGF- $\beta R$ signaling has also been implicated for the development and maintenance of microglia ${ }^{50}$. Yet, microglia-specific deletion of TGF- $\beta$ R revealed its role in maintaining the homeostatic phenotype of adult microglia, but not their survival.

Sall1 controls microglia fate by regulating their unique gene expression profile, allowing them to exert their 'house-keeping' functions critical for homeostasis of the adult CNS. We show that deletion of Sall1 in microglia specifically leads to a genetic as well as phenotypic activation of microglia that results in a disrupted CNS homeostasis as reflected by dysregulated neurogenesis. Taken together our data provide a new approach of specifically targeting microglia, allowing for detailed analysis of microglia biology and targeted modulation in vivo. 
Closing remarks 


\section{Materials and Methods}

\subsection{Mice and animal experiments}

Mice were housed in individually ventilated cages under specific pathogen-free conditions. Conventional C57BL/6 and C57BL/6-CD45.1 mice (wt) were purchased from Janvier Labs. Sall1 ${ }^{\mathrm{CreER}}$, Sall $1^{\mathrm{fl} / \mathrm{fl}}$ and Sall1 ${ }^{\mathrm{GFP}}$ mice were described previously 95,104,118,122. Cx3cr1 ${ }^{\text {CreER }}$ were kindly provided by Steffen Jung (Weizmann Institute of Science, Israel) ${ }^{19}$. Csf1 $r^{\text {fl/fl }}$ mice were kindly provided by Jeffrey Pollard (Albert Einstein College of Medicine, New York $)^{123}$. Tgfbr2 $2^{\mathrm{fl} / \mathrm{fl}}, \mathrm{Cx} 3 \mathrm{cr} 1^{\mathrm{GFP}}$, iDTR, R26-yfp and R26-rfp mice were bred in-house ${ }^{98,124,125}$.

To perform embryo studies at a specific age, timed matings were set up: male and female mice were mated over night, and the day after was counted as embryonic day $(E)$ 0.5. At the day of analysis pregnant females were euthanized by $\mathrm{CO}_{2}$ inhalation, embryos were removed and euthanized by decapitation.

Experimental autoimmune encephalomyelitis (EAE) was induced as described previously ${ }^{126}$. Briefly, mice were immunized subcutaneously with $200 \mu \mathrm{g}$ of MOG $_{35-55}$ peptide (MEVGWYRSPFS-RVVHLYRNGK; GenScript) emulsified in CFA (Difco) in a total volume of $200 \mu$ land two intraperitoneal injections of $200 \mathrm{ng}$ Pertussis toxin (Sigma) on day 0 and 2. Clinical disease was scored as follows: 0 , no detectable signs of EAE; 0.5 , distal limp tail; 1 , complete limp tail; 1.5 , limp tail and hind limb weakness; 2, unilateral partial hind limb paralysis; 2.5, bilateral partial hind limb paralysis; 3, complete bilateral hind limb paralysis; 3.5, complete hind limb paralysis and unilateral forelimb paralysis; 4 , total paralysis of fore and hind limbs (score $\geq 4$, to be killed); 5 , death.

Bone marrow (BM) chimeras were generated as previously described ${ }^{127}$. In brief, recipient mice were lethally irradiated with a split-dose of $2 \times 550$ rad about $15 \mathrm{~h}$ apart using a RS 2000 Biological Research X-ray Irradiator (Rad Source Technologies) and reconstituted i.v. with $2-5 \times 10^{6} \mathrm{BM}$ cells isolated from the respective gender-matched donor mice. Mice were treated with $0.1 \%$ Borgal (Intervet) in drinking water for two weeks to prevent bacterial infections. Reconstitution of recipient mice was monitored by tail bleeding and flow cytometry at 6 weeks post transfer. 
In order to enable Cre recombination in Tamoxifen-inducible CreER mouse strains, Tamoxifen (Sigma) was dissolved in corn oil to a concentration of $25 \mathrm{mg} / \mathrm{ml}$ and was administered i.p. (2.5 mg) or via o.g. (5 mg). Alternatively, mice were fed ad libitum with a diet containing 400 ppt tamoxifen citrate (Harlan) for 4 - 6 weeks.

The small molecule kinase inhibitor PLX5622 to block Csf-1R signaling was provided by Plexxikon and was administered ad libitum via a diet containing 1,200 $\mathrm{mg} / \mathrm{kg}$.

For systemic inflammation via LPS treatment $100 \mu \mathrm{g}$ LPS (Sigma) were administered i.p. and animals were euthanized $24 \mathrm{~h}$ after the injections.

To induce targeted cell death in iDTR mouse strains $500 \mathrm{ng}$ DTx (Sigma) were administered i.p.

All animal experiments performed in this study were approved by the Swiss cantonal veterinary office (licenses 86/2012 and 100/2015).

\subsection{DNA isolation and genotyping}

Biopsies were collected from toe tips or ears of mice and lysed for approximately 1 h at $95^{\circ} \mathrm{C}$ in $50 \mathrm{mM} \mathrm{NaOH}$ solution. $1 \mathrm{M} \mathrm{TrisHCl} \mathrm{pH} 8.5$ was added to neutralize the reaction. Samples were centrifuged at $10^{\prime} 000 \mathrm{rpm}$ to pellet the remaining tissue debris and the supernatant was used for DNA analysis.

For genotyping $1 \mu \mathrm{l}$ of DNA template and $0.5 \mu \mathrm{l}$ of each primer $(10 \mu \mathrm{M}$, Thermo Fisher Scientific) was used in 1x Hot Fire Pol Blend master mix (Solis BioDyne). PCR amplification was performed on a C1000 Thermal Cycler (BioRad) as indicated below and PCR products were analyzed in a $1.5 \%$ Agarose (Biocompare) gel containing GelRed (Biotium) for DNA visualization using the Alphalmager 2200 with the AlphaEaseFC software. 
PCR protocol:

\begin{tabular}{|l|l|l|l|}
\hline Task & Temperature $\left({ }^{\circ} \mathrm{C}\right)$ & Time $(\mathrm{sec})$ & Cycles \\
\hline initial denaturation & 95 & 900 & \\
\hline denaturation & 94 & 30 & \multirow{2}{*}{35} \\
\cline { 1 - 2 } annealing & 58 & 45 & \\
\hline extention & 72 & 60 & \\
\hline final extension & 72 & 420 & \\
\hline
\end{tabular}

List of primers and gene products:

\begin{tabular}{|c|c|c|}
\hline Gene & Primer sequence ( $5^{\prime}$ to $\left.3^{\prime}\right)$ & PCR product (bp) \\
\hline Sall $1^{f l}$ & $\begin{array}{l}\text { 1: CCT CTG CCC GAG AGA TCG } \\
\text { 2: GGC GCG TCT GAT TTT ATT TC } \\
\text { 3: AGG AAC ACT CAC GAA ATG GG }\end{array}$ & $\begin{array}{l}\text { wt: } 220 \\
\text { mut: } 280\end{array}$ \\
\hline $\begin{array}{l}\text { Sall1 } 1^{\mathrm{GFP}} / \\
\text { Sall } 1^{\text {CreER }}\end{array}$ & $\begin{array}{l}\text { 1: CAA CTT GCG ATT GCC ATA AA } \\
\text { 2: GCG TTG GCT ACC CGT GAT AT } \\
\text { 3: AGC TAA AGC TGC CAG AGT GC }\end{array}$ & $\begin{array}{l}\text { wt: } 288 \\
\text { mut: } 350\end{array}$ \\
\hline $\begin{array}{l}\text { General } \\
\text { Cre }\end{array}$ & $\begin{array}{l}\text { 1: GCG GTC TGG CAG TAA AAA CTA TC } \\
\text { 2: GTG AAA CAG CAT TGC TGT CAC TT } \\
\text { 3: CTA GGC CAC AGA ATT GAA AGA TCT } \\
\text { 4: GTA GGT GGA AAT TCT AGC ATC ATC C }\end{array}$ & $\begin{array}{l}\text { wt: } 324 \\
\text { mut: } 100\end{array}$ \\
\hline $\begin{array}{l}\text { R26-yfp / } \\
\text { R26-rfp / } \\
\text { R26-dtr }\end{array}$ & $\begin{array}{l}\text { 1: AAG ACC GCG AAG AGT TTG TC } \\
\text { 2: AAA GTC GCT CTG AGT TGT TAT } \\
\text { 3: GGA GCG GGA GAA ATG GAT ATG }\end{array}$ & $\begin{array}{l}\text { wt: } 600 \\
\text { mut: } 320\end{array}$ \\
\hline
\end{tabular}




\begin{tabular}{|c|c|c|}
\hline Csf1r ${ }^{f l}$ & $\begin{array}{l}\text { 1: ACC CAG AGC CCC CAC AGA TA } \\
\text { 2: GCC ACC ATG TGT CCG TGC TT }\end{array}$ & $\begin{array}{l}\text { wt: } \quad 445 \\
\text { mut: } \quad 306\end{array}$ \\
\hline Tgfbr $2^{f \mid}$ & $\begin{array}{l}\text { 1: TAT GGA CTG GCT GCT TTT GTA TTC } \\
\text { 2: TGG GGA TAG AGG TAG AAA GAC ATA }\end{array}$ & $\begin{array}{l}\text { wt: } \quad 422 \\
\text { mut: } \quad 575\end{array}$ \\
\hline
\end{tabular}

\subsection{Leukocyte isolation from CNS}

Mice were sacrificed by $\mathrm{CO}_{2}$ inhalation and intracardially perfused with approximately $20 \mathrm{ml}$ PBS. The brain was carefully removed from the skull and the spinal cord was flushed out by hydrostatic pressure. When indicated, choroid plexus was manually separated from the ventricle areas of the CNS using fine Dumont forceps (5) and a binocular. Brain and spinal cord were pooled and cut into small pieces using scissors, followed by a tissue digest in $0.4 \mathrm{mg} / \mathrm{ml}$ collagenase type IV (from Clostridium histolyticum, Sigma) in HBSS (containing $\mathrm{Ca}^{2+} / \mathrm{Mg}^{2+}$, Invitrogen) supplemented with $10 \%$ FCS (Biochrom AG) for $45-60$ min at $37^{\circ} \mathrm{C}$ while agitating. Digested tissues were passed through an $18 \mathrm{G}$ syringe and filtered through a $70 \mu \mathrm{m}$ cell strainer to obtain a homogeneous cell suspension. After washing, the cell suspension was further enriched using a $30 \%$ Percoll (GE Healthcare) continuous gradient, centrifuged at $4^{\circ} \mathrm{C}$ for $30 \mathrm{~min}$ at $10^{\prime} 800 \mathrm{rpm}$ with low brakes. The myelin in the upper Percoll layer was removed and the remaining cell suspension was washed and transferred into $1.5 \mathrm{ml}$ tubes for further analysis.

\subsection{Leukocyte isolation from other organs}

Mice were sacrificed by $\mathrm{CO}_{2}$ inhalation and intracardially perfused with approximately $20 \mathrm{ml}$ PBS. Organs of interest were harvested, cut into small pieces and digested for $45 \mathrm{~min}$ at $37^{\circ} \mathrm{C}$ in collagenase solution as described above. Digested tissues were passed through an $18 \mathrm{G}$ syringe and filtered through a $70 \mu \mathrm{m}$ cell strainer to obtain a homogeneous cell suspension.

For blood, spleen and kidney, red blood cells were lysed in red blood cell lysis buffer (4.15 $\mathrm{g} \mathrm{NH}_{4} \mathrm{Cl}, 0.55 \mathrm{~g} \mathrm{KHCO}_{3}, 0.185 \mathrm{~g} \mathrm{EDTA}$ in $500 \mathrm{ml} \mathrm{ddH} \mathrm{H}_{2} \mathrm{O}$ ) for $5 \mathrm{~min}$ at RT. Samples were washed with PBS before further processing. This step was repeated if necessary. 
Liver samples were enriched in a continuous 30\% Percoll gradient, centrifuged for 30 minutes at $1700 \mathrm{rpm}$ at room temperature without brakes. After fat and supernatant in the upper layers were removed, red blood cell lysis was performed on the pellet containing the leukocytes as described before. Cells were washed with PBS and filtered using a $70 \mu \mathrm{m}$ cell strainer to obtain a single cell suspension.

As skin samples ears were used, which were split into dorsal and ventral parts using forceps and incubated at $37^{\circ} \mathrm{C}$ for $90 \mathrm{~min}$ in $2.4 \mathrm{mg} / \mathrm{ml}$ of Dispase (Sigma) diluted in HBSS (containing $\mathrm{Ca}^{2+} / \mathrm{Mg}^{2+}$, Invitrogen). Afterwards dermis and epidermis were separated and both were cut separately into small pieces and digested in collagenase solution as described above for $2 \mathrm{~h}$. Cells were washed with PBS and filtered using a $70 \mu \mathrm{m}$ cell strainer to obtain a single cell suspension.

\subsection{Flow cytometry and cell sorting}

Single cell suspensions were stained for $20-30 \mathrm{~min}$ at $4{ }^{\circ} \mathrm{C}$ in an appropriate amount of PBS or FACS buffer (PBS with $2 \%$ FCS and $0.01 \% \mathrm{NaN}_{3}$ ). Before analysis the cells were washed once with PBS and filtered through a $70 \mu \mathrm{m}$ cell strainer again. Flow cytometric analysis was performed using a FACSCanto II or LSRII Fortessa flow cytometer and FACSDiva software (BD Biosciences). Cell sorting was carried out using a FACSAria III (BD Biosciences) using an $85 \mu \mathrm{m}$ nozzle, with a purity of $>95 \%$. Data analysis was performed using FlowJo 9.9.x or 10.0.x software (TreeStar). Fluorochrome-conjugated monoclonal antibodies (mAb) specific for mouse I-A/I-E (clone M5/114.15.2), CD11b (clone M1/70), CD11C (clone N418), CD45 (clone 30-F11), CD45.1 (clone A20), CD45.2 (clone 104), CD115 (clone AFS98), Ly6C (clone AL-21 or HK1.4), Ly6G (clone 1A8), Gr1 (clone RB6-8C4), Siglec-H (clone 551), CD3 (clone 17A2), CD45R (clone RA3-6B2), Siglec-1 (clone 3D6.112), CD64 (clone X54-5/7.1), Siglec-F (clone E50-2440), CD103 (clone 2E7), Ki67 (clone SOLA15), GLAST (clone ACSA-1), MOG (clone 818c5), Lyve1 (clone ALY7), MerTK (clone DS5MMER), CD206 (clone MR5D3), CD31 (clone 390), EpCam (clone G8.8), CD4 (clone GK1.5) purchased either from $\mathrm{BD}$ Biosciences, eBioscience, or Biolegend. Anti-F4/80 (clone Cl:A3-1) mAb was purchased from $\mathrm{AbD}$. Prior to detailed analysis cells were always gated on single, live cells using FSC and SSC, dead cells were excluded with the live/dead Fixable 
Aqua staining kit (life technologies). Staining for Ki67 was performed using Foxp3 Staining Buffer Set (eBiosciences) according to the manufacturer's protocol.

\section{6 t SNE dimensionality reduction}

A concatenated FCS file of single, live CNS cells (10'000 cells each) and separately isolated choroid plexus cells (ca 1'500 cells each) from 3 Sall $1^{\mathrm{GFP} /+}$ mice was created using FlowJo $X$ and uploaded to cytobank (http://fluidigm.cytobank.org). t-SNE dimensionality reduction was calculated based on all surface markers in the staining panel, excluding GFP (Sall1). Different CNSresident populations were subsequently gated manually as shown in Figure 13a and overlayed onto the two-dimensional t-SNE map. Expression heat-maps of the respective surface markers and Sall1 (GFP) where overlayed onto the respective tSNE maps.

\subsection{RNA isolation and quantitative rt-PCR}

Cells of interest were sorted into $1.5 \mathrm{ml}$ tubes and centrifuged at $5000 \mathrm{rpm}$ for 10 min at $4^{\circ} \mathrm{C}$. Supernatant was discarded and the cell pellet was lysed in RLT plus buffer (Qiagen) supplemented with $1 \% \beta$-mercaptoethanol (Sigma). RNA isolation was performed using the RNeasy Micro Plus Kit (Qiagen) according to the manufacturer's instructions. RNA concentration was estimated using a NanoDrop 1000 spectrophotometer (Thermo Scientific).

For first strand cDNA synthesis $10 \mu \mathrm{l}$ RNA were added to a $10 \mu \mathrm{l}$ reaction mix containing M-MLV reverse transcriptase (200 U, Invitrogen), dNTPs (0.5 mM, Invitrogen), First strand buffer (1x, Invitrogen), DTT (10 mM, Invitrogen), oligo (dt) primers (10 ng, PeproTech) and RNasin Plus RNase Inhibitor (Promega) recombinant ribonuclease inhibitor (40 U, Thermo Fischer Scientific). Samplereaction mix was incubated $10 \mathrm{~min}$ at $\mathrm{RT}, 50 \mathrm{~min}$ at $37^{\circ} \mathrm{C}$ for reverse transcription, and $15 \mathrm{~min}$ at $70^{\circ} \mathrm{C}$ to inactivate the transcriptase. cDNA was diluted in water depending on the starting amount of RNA.

For quantitative real-time PCR $2.5 \mu \mathrm{l}$ of cDNA template was mixed with $0.25 \mu \mathrm{l}$ of the respective forward and reverse primers (indicated below), $3.25 \mu \mathrm{H} 2 \mathrm{O}$ and $6.25 \mu \mathrm{l}$ iTaq Universal SYBR Green Supermix (BioRad). Each sample was measured in triplicates on a CFX384 Touch Real-Time PCR Detection System 
(Bio-Rad). Cycle threshold ( $\mathrm{Ct}$ ) values of gene of interest were normalized to the house keeping gene RNA polymerase II (Pol2) and relative gene expression was plotted as $2^{-\Delta C q}$.

\begin{tabular}{|l|l|}
\hline Gene & Primer sequence (5' to 3') \\
\hline Sall1 & $\begin{array}{l}\text { Fwd: CCC GTG AGC GGC TGA TGT TTG AG } \\
\text { Rev: TGG GGC GAC TTG GTT GAC CCT }\end{array}$ \\
\hline Csfr1 & $\begin{array}{l}\text { Fwd: GAC ATC CCC AGT TCT GCT CC } \\
\text { Rev: ACC TCG CCG CTA GAT CCT TC } \\
\text { Fwd: CGC CGA AGT GGG ATT CAA CG }\end{array}$ \\
\hline Tgfbr2 & $\begin{array}{l}\text { Fwd: AAC GAC TTG ACC TGT TGC CTG T } \\
\text { Rev: CTT CCG GGG CCA TGT ATC TT }\end{array}$ \\
\hline I 1b & $\begin{array}{l}\text { Fwd: GAT CCA CAC TCT CCA GCT GCA } \\
\text { Rev: CAA CCA ACA AGT GAT ATT CTC CAT G }\end{array}$ \\
\hline Tnfa & $\begin{array}{l}\text { Fwd: CAT CTT CTC AAA ATT CGA GTG ACA A } \\
\text { Rev: TGG GAG TAG ACA AGG TAC AAC CC }\end{array}$ \\
\hline Cxcl10 & $\begin{array}{l}\text { Fwd: CTG CCG TCA TTT TCT GCC TC } \\
\text { Rev: CAC TGG CCC GTC ATC GAT AT }\end{array}$ \\
\hline Pol2 & $\begin{array}{l}\text { Fwd: CTG GTC CTT CGA ATC CGC ATC } \\
\text { Rev: GCT CGA TAC CCT GCA GGG TCA }\end{array}$ \\
\hline
\end{tabular}




\subsection{Next generation sequencing}

For next generation sequencing (NGS) microglia of Sall ${ }^{\text {CreER/fl }}$ and control Sall1 $1^{\mathrm{fl/fl}}$ mice were sorted and total RNA was isolated using the RNeasy micro plus kit (Qiagen). 2 ng RNA were amplified using the Ovation ${ }^{\circledR}$ Single Cell RNA-seq System kit (Nugen) according to the manufacturer's protocol. NGS and bioinformatic analysis was performed by the Functional Genomics Center Zurich (FGCZ) using the HiSeq 2500 v4 System (Illumina). Quality control included fastqc and DESeq2 analysis. GO pathway analysis was performed using the MetaCore ${ }^{\mathrm{TM}}$ software (Thomson Reuters) and visualization was done using the MultiExperiment Viewer (TM4) and Prism (GraphPad).

\subsection{Immunohistochemistry}

Mice were euthanized with $\mathrm{CO}_{2}$ and intracardially perfused with approximately 20 $\mathrm{ml}$ PBS. The CNS was carefully extracted and post-fixed in $4 \%$ paraformaldehyde (Kantonsaptheke, Morphisto) for $24 \mathrm{~h}$. The samples were preserved in $30 \%$ sucrose (Sigma) solution in PBS for $24-48 \mathrm{~h}$ prior to embedding in OCT cryo embedding medium (Medite) on dry ice. Cryo-sections were stored at $-80^{\circ} \mathrm{C}$ until further processing. For immunohistochemistry, cryo-embedded CNS samples were cut on a HYRAX C60 cryostat microtome at $14 \mu \mathrm{m}$ for direct mounting on glass slides or at $40 \mu \mathrm{m}$ for free-floating sections in a 24-well plate with $1 \mathrm{ml}$ PBS per well supplemented with $0.1 \% \mathrm{NaN}_{3}$.

Directly mounted samples were washed once with PBS to remove the remaining OCT medium. CNS samples were blocked for $1 \mathrm{~h}$ at RT with blocking buffer containing PBS, $10 \%$ normal goat serum (NGS, Thermo Fisher Scientific) or BSA (Sigma) and $0.5 \%$ Triton-x100 (Sigma). The primary antibodies were diluted in PBS supplemented with $4 \%$ NGS or BSA and $0.1 \%$ Triton-x 100 as listed below and incubated for $2 \mathrm{~h}$ at RT or overnight at $4^{\circ} \mathrm{C}$. After thorough washing with PBS, samples ere incubated with different fluorochrome-conjugated secondary antibodies (Invitrogen) directed against the host IgG. Samples were washed again and covered with mounting medium containing DAPI (Dianova). 
Materials and Methods

\begin{tabular}{|l|l|l|l|}
\hline Antigen & Host species & Company & dilution $(1: \mathrm{x})$ \\
\hline Calbindin & rabbit & Abcam & 200 \\
\hline CD31 & rabbit & BD Biosciences & 200 \\
\hline DCX & rabbit & Cell Signaling & 200 \\
\hline F4/80 & rat & AbD Serotec & 200 \\
\hline GFAP & rat & Invitrogen & 500 \\
\hline GFAP & mouse & Sigma & 500 \\
\hline GFP & rabbit & Abcam & 5000 \\
\hline GFP & chicken & Aves Labs & 500 \\
\hline Iba-1 & rabbit & WAKO & 500 \\
\hline Ki67 & rat & eBioscience & 200 \\
\hline MBP & mouse & Millipore & 300 \\
\hline NeuN & mouse & Millipore & 300 \\
\hline Olig2 & rabbit & Sigma & 500 \\
\hline S100B & rabbit & Abcam & 200 \\
\hline
\end{tabular}

Images were acquired on a Confocal Laser Scanning Microscope SP5 (Leica) and analysis was performed using the Imaris software (Bitplane) and Fiji (ImageJ, GNU General Public License).

For 3D reconstruction, microglia were recorded with a $63 x$ magnification and 3times zoom in $Z$ stacks of $0.17 \mu$ m steps in $z$ direction, $512 \times 512$ pixel resolution. Images were analyzed using an Imaris-based automatic reconstruction (Bitplane). 


\subsection{Phagocytosis assay}

Primary microglial cells were isolated ex vivo from Sall $1^{\text {CreER/fl }}$ and control Sall1 $1^{\mathrm{fllfl}}$ mice as described above for leukocyte isolation from CNS. Cells were plated into a 48-well plate with PBS supplemented with $5 \%$ FCS and cultured for $4 \mathrm{~h}$ with pHodro Red Zymosam Bioparticles (Thermo Fisher Scientific) at $37^{\circ} \mathrm{C}$. As negative control cells were incubated with Cytochalasin D (Sigma) 30 min prior to the assay to block phagocytosis. For flow cytometric analysis, cells were detached using Accutase (Thermo Fisher Scientific), washed once with PBS and stained as described before. For immunohistochemistry, cells were directly plated on poly-LLysine coated glass coverslips at the beginning of the assay, washed once with PBS and counter stained with Iba-1 as described before. Maximal excitation/emission of pHrodo Red was measured at 560/585 nm.

\subsection{Multiplex immunoassay}

For cytokine measurements, serum as well as CNS, spleen, kidney, liver and heart were isolated from Sall $1^{\mathrm{CreER} / \mathrm{fl}}$ and control Sall $1^{\mathrm{fl} / \mathrm{fl}}$ mice. For whole tissue lysates 80-110 mg tissue were homogenized in Cell Lysis Buffer (Cell Signaling) using the a TissueLyser (Qiagen) for $2.5 \mathrm{~min}$ at $25 \mathrm{~Hz}$. Samples were rested on ice for 30 min, sonicated twice for 10 seconds at $35 \%$ maximal amplitude and centrifuged at 12 '000 rpm for $10 \mathrm{~min}$. Supernatant was used for further analysis. GM-Csf, IFN-y, IL-10, IL-12p70, IL-1 $\beta$, IL-2 IL-6 and TNF- $\alpha$ were measured on a 8-plex electrochemiluminescence detection assay and read on the MSD QuickPlex SQ 120 (both Meso Scale Discovery, Gaithersburg, MD).

\subsection{Statistical analysis}

Mean values, SEM values, and p-values were calculated and plotted with Prism 5 software (GraphPad). Two-tailed unpaired t-test was performed to determine statistical significance between two groups. Welsh's correction was applied in case of significantly different variances. For three or more groups one-way ANOVA with Dunnett or Bonferroni post test was performed. p-Values below 0.05 were considered significantly different $\left({ }^{*} \mathrm{P}<0.05\right.$, $\left.{ }^{* *} \mathrm{P}<0.01,{ }^{* * *} \mathrm{P}<0.001\right)$. Data is displayed as mean \pm standard error of the mean (SEM). 


\section{Acknowledgements}

First and foremost I would like to thank my parents Ute, Thomas and Bertram for their constant love and support throughout all the years. Without them I would not be standing where I am today. They always had my back, believed in me and encouraged me to go my own way. I'm very grateful for their well-meant advice even if I did not want to hear it at times. They taught me to speak up for myself and never forget who I am. And they reminded me of what I already achieved when I lost track.

Along with this, I would like to thank all the rest of my family and my boyfriend for taking great pride in my achievements and supporting me along the way. I am more than grateful to know that I could rely on each and everyone of them when necessary. With their positive spirit and unbreakable believe they helped me build a strong and independent personality. They all make it impossible to lose track of what really counts in life and for that I am infinitely thankful.

Without any doubt my biggest scientific acknowledgement goes to Melanie Greter, who gave me the opportunity to work on this exciting, cutting edge project. This sentence might sound a bit worn out and routine, but it is actually true. Melanie is a brilliant scientist, providing valuable inputs and leading the project in the right way. She managed to see the good in every dataset while at the same time not losing a critical point of view. Melanie was always supportive in scientific as well as personal matters, which I highly appreciate. I definitely learned a lot in the past four years on a wide spectrum that will accompany me throughout my future career and life.

I would also like to thank my $\mathrm{PhD}$ committee members Burkhard Becher, Christian Münz and Frank Heppner for their important support during this $\mathrm{PhD}$ project and the friendly atmosphere they created. They helped a lot in discussing critical aspects of the work and in prioritizing the individual parts of the story. Burkhard particularly put a lot of effort and "selling" support into our publication to push it to the point where it ended up now. I appreciate that a lot.

I am also very grateful for the valuable support by different core facilities at the University of Zurich and the people involved, including the Center for Microscopy and Image Analysis, the Functional Genomics Center and most of all the Flow 
Core Facility with Vinko Tosevski and Florian Mair. Without the support of these facilities the PhD work presented here would not be of such high quality as it is.

No research project could be managed without the support of great colleagues. I would like to thank all our lab technicians, especially Jennifer Jarberg and Sabrina Nemetz, for all the work they did in the background without even being noticed, for the help with experiments, for taking care of "super-special, super-urgent" orders and for always having an open ear and discussing technical issues. A big acknowledgement also goes to the numerous animal caretakers and the coordinators at the Laboratory Animal Service Center, for keeping the animal facility running and providing the basis for all experiments. In addition, I would like to thank Ines Scholz and our additional coordinators for their efficient and reliable work concerning all administrative issues. Along the same lines I would like to acknowledge the Microbiology \& Immunology PhD program of the Life Science Zurich Graduate School for providing a great scientific and social framework to this doctorate thesis.

One of the biggest 'thank you's goes my fellow research colleagues at the Institute of Experimental Immunology, especially Kathrin, Sabi and Flo, for critical discussions of experiments and various technical approaches. But even more so, for sharing ups and downs of the past years, for supporting and understanding me during stressful periods, and for lifting up my spirits when necessary with running, hiking, biking or going out for a drink.

Last but not at all least I would like to thank all my friends who supported me along the way, a way that not just started in Zurich but far before that. I'm very grateful for each and every one of them no matter how close or far they are. They reminded me of what really counts in life, they shared my joy even if they themselves had difficult times and they lifted me up when I was upset. I cannot put in words how much I value having those friends in my life. 


\section{Curriculum Vitae}

Personal data:

Name: Anne Buttgereit

Birth date: 15.02.1989, Suhl, Germany

Nationality: German

Address: $\quad$ Viktoriastrasse 61

8050 Zurich, Switzerland

Phone: $\quad$ +41(0)791758681

E-mail: buttgereit@immunology.uzh.ch

\section{Education:}

2012-2016: PhD studies in the Microbiology and Immunology program of the Life Science Zurich Graduate School (LSZGS), Zurich, Switzerland, Thesis title: "Sall1 is a transcriptional regulator defining microglia identity and function", Supervisor: Prof. Dr. Melanie Greter, Institute of Experimental Immunology, Research Unit of Myeloid Cell Development, University of Zurich, Switzerland

2010-2012: M.Sc. studies in Molecular Medicine, University Ulm, Germany, Thesis title: "Role of PPARס in neuronal function and neurodegeneration", Supervisor: Albert R. La Spada, MD, PhD, Division of Genetics, Department of Pediatrics and Cellular \& Molecular Medicine, University of California San Diego, USA

2007-2010: B.Sc. studies in Molecular Medicine, University Ulm, Germany, Thesis title: "Influence of the PPAR-signaling system on the activation of microglial cells", Supervisor: Prof. Dr. Anke Witting, Department of Neurology, University UIm

1999-2007: High school, Heinrich-Ehrhardt Gymnasium, Zella-Mehlis, Germany 


\section{Academic commitment:}

May 2016: Data Carpentry Workshop in R, University of Zürich and sciCORE Center for Scientific Computing Basel, Switzerland

2015/2016: Supervision of a master student in Biology / Neurosciences, University of Zurich, Switzerland

Nov 2014: Advanced course in flow cytometry, University of Zürich, Switzerland 2013-2016: Teaching of student courses for Immunology and Medical Immunology, University of Zurich \& ETH, Zurich, Switzerland

June 2013: Introductory course in Laboratory Animal Science (FELASA approved), University of Zürich, Switzerland

May 2013: Project management for research, University of Zürich, Switzerland

July 2011: Local organizing team of the FENS/IBRO Summer School "Metabolic Aspects of Chronic Brain Diseases", University UIm, Günzburg, Germany

2008-2011: Organization of the first annual "Day of Molecular Medicine“, founder member of the student body of the faculty of Molecular Medicine, University Ulm, Germany

\section{Language \& technical kills:}

Language: German (native), English (fluent), French (basic knowledge)

Laboratory advanced knowledge in high-dimensional flow cytometry, cell

Techniques: sorting, RNA sequencing and immunohistochemistry; basic skills in CyTOF extensive experience in mouse handling (maintenance and monitoring, disease models, embryonic studies) and in standard laboratory techniques (ex vivo cell isolation, primary tissue culture, qPCR, ELISA, Western Blot)

Analysis \& profound knowledge in Microsoft Office, Adobe Creative Suite,

Presentation: TreeStar FlowJo, GraphPad Prism, Bitplane Imaris; basic skills in R 


\section{Scholarships \& grants:}

2016: MIM Travel Grant supported by the Microbiology and Immunology PhD program, ETH and University Zürich

2015: Congress-scholarship for young researchers awarded by the Swiss Society of Allergology and Immunology (SSAI)

2011/12: Biomedical Sciences Exchange Program (BMEP) funded by the International Academy of Life Sciences (IALS) and the German Academic Exchange Service (DAAD)

2008-2010: Scholarship awarded by the University UIm for students with outstanding performances

\section{International conferences \& meetings:}

Sept $201612^{\text {th }} \& 13^{\text {th }}$ International Society of Neuroimmunology Congress,

\& Nov 2014: Jerusalem, Israel, (oral presentation); and Mainz, Germany, July 2015: $\quad 12^{\text {th }}$ European Meeting on Glial Cells in Health and Disease, Bilbao, Spain;

Apr $201325^{\text {th }} \& 26^{\text {th }}$ Wolfsberg meeting of Swiss PhD students in Immunology \& Apr 2012: Ermatingen, Switzerland; oral / poster presentation

\section{Publications:}

Buttgereit A, Lelios I, Yu X, Vrohlings M, Krakoski NR, Gautier EL, Nishinakamura $\mathrm{R}$, Becher $\mathrm{B}$ and Greter $\mathrm{M}$ : "Sall1 is a transcriptional regulator defining microglia identity and function", Nature Immunology, Nov 2016

Dickey AS, Pineda VV, Tsunemi T, Liu PP, Miranda HC, Gilmore-Hall SK, Lomas N, Sampat KR, Buttgereit A, Manalang Torres MJ, Flores AL, Arreola M, Arbez N, Akimov SS, Gaasterland T, Lazarowski ER, Ross CA, Yeo GW, Sopher BL, Magnuson GK, Pinkerton AB, Masliah E and La Spada AR: "PPAR- $\delta$ is repressed in Huntington's disease, is required for normal neuronal function and can be targeted therapeutically", Nature Medicine, Jan 2016 


\section{References}

1. Chaplin, D. D. Overview of the immune response. J. Allergy Clin. Immunol. 125, S3-23 (2010).

2. Junt, T., Scandella, E. \& Ludewig, B. Form follows function: lymphoid tissue microarchitecture in antimicrobial immune defence. Nature Reviews Immunology 8, 764-775 (2008).

3. Dranoff, G. Cytokines in cancer pathogenesis and cancer therapy. Nat. Rev. Cancer 4, 11-22 (2004).

4. Parkin, J. \& Cohen, B. An overview of the immune system. Lancet 357, 1777-1789 (2001).

5. Gasteiger, G. \& Rudensky, A. Y. Interactions between innate and adaptive lymphocytes. Nature Reviews Immunology 14, 631-639 (2014).

6. Jonsson, A. H. \& Yokoyama, W. M. Natural killer cell tolerance licensing and other mechanisms. Adv. Immunol. 101, 27-79 (2009).

7. Cao, X. Self-regulation and cross-regulation of pattern-recognition receptor signalling in health and disease. Nature Reviews Immunology 16, 35-50 (2016).

8. Iwasaki, A. \& Medzhitov, R. Control of adaptive immunity by the innate immune system. Nat Immunol 16, 343-353 (2015).

9. O'Neill, L. A. J., Golenbock, D. \& Bowie, A. G. The history of Toll-like receptors - redefining innate immunity. Nature Reviews Immunology 13, 453-460 (2013).

10. Franchi, L., Warner, N., Viani, K. \& Nuñez, G. Function of Nod-like receptors in microbial recognition and host defense. Immunological Reviews 227, 106-128 (2009).

11. Dunkelberger, J. R. \& Song, W.-C. Complement and its role in innate and adaptive immune responses. Cell Res. 20, 34-50 (2010).

12. Fujita, T. Evolution of the lectin-complement pathway and its role in innate immunity. Nature Reviews Immunology 2, 346-353 (2002).

13. Germain, R. N. T-cell development and the CD4-CD8 lineage decision. Nature Reviews Immunology 2, 309-322 (2002).

14. Sakaguchi, S., Yamaguchi, T., Nomura, T. \& Ono, M. Regulatory T cells and immune tolerance. Cell 133, 775-787 (2008).

15. LeBien, T. W. \& Tedder, T. F. B lymphocytes: how they develop and function. Blood 112, 1570-1580 (2008).

16. van Furth, R. et al. [Mononuclear phagocytic system: new classification of macrophages, monocytes and of their cell line]. Bull. World Health Organ. 47, 651-658 (1972).

17. Guilliams, M. et al. Dendritic cells, monocytes and macrophages: a unified nomenclature based on ontogeny. Nature Reviews Immunology 14, 571578 (2014).

18. Hettinger, J. et al. Origin of monocytes and macrophages in a committed progenitor. Nat Immunol 14, 821-830 (2013).

19. Yona, S. et al. Fate Mapping Reveals Origins and Dynamics of Monocytes and Tissue Macrophages under Homeostasis. Immunity 1-13 (2012). doi:10.1016/j.immuni.2012.12.001

20. Mellman, I. \& Steinman, R. M. Dendritic cells: specialized and regulated antigen processing machines. Cell 106, 255-258 (2001).

21. Corcoran, L. et al. The lymphoid past of mouse plasmacytoid cells and 
thymic dendritic cells. J. Immunol. 170, 4926-4932 (2003).

22. Hashimoto, D., Miller, J. \& Merad, M. Dendritic cell and macrophage heterogeneity in vivo. Immunity 35, 323-335 (2011).

23. Villadangos, J. A. \& Young, L. Antigen-presentation properties of plasmacytoid dendritic cells. Immunity 29, 352-361 (2008).

24. Orkin, S. H. \& Zon, L. I. Hematopoiesis: an evolving paradigm for stem cell biology. Cell 132, 631-644 (2008).

25. Lin, Y., Yoder, M. C. \& Yoshimoto, M. Lymphoid progenitor emergence in the murine embryo and yolk sac precedes stem cell detection. Stem Cells Dev. 23, 1168-1177 (2014).

26. Hoeffel, G. et al. C-myb(+) erythro-myeloid progenitor-derived fetal monocytes give rise to adult tissue-resident macrophages. Immunity 42, 665-678 (2015).

27. Hashimoto, D. et al. Tissue-Resident Macrophages Self-Maintain Locally throughout Adult Life with Minimal Contribution from Circulating Monocytes. Immunity 38, 792-804 (2013).

28. Dai, X.-M. et al. Targeted disruption of the mouse colony-stimulating factor 1 receptor gene results in osteopetrosis, mononuclear phagocyte deficiency, increased primitive progenitor cell frequencies, and reproductive defects. Blood 99, 111-120 (2002).

29. Greter, M. et al. Stroma-Derived Interleukin-34 Controlsthe Development and Maintenance of Langerhans Cells and the Maintenance of Microglia. Immunity 1-11 (2012). doi:10.1016/j.immuni.2012.11.001

30. Lavin, Y. et al. Tissue-Resident Macrophage Enhancer Landscapes Are Shaped by the Local Microenvironment. Cell 159, 1312-1326 (2014).

31. Gosselin, D. et al. Environment drives selection and function of enhancers controlling tissue-specific macrophage identities. Cell 159, 1327-1340 (2014).

32. Gautier, E. L. et al. Gene-expression profiles and transcriptional regulatory pathways that underlie the identity and diversity of mouse tissue macrophages. Nat Immunol 13, 1118-1128 (2012).

33. Merad, M., Ginhoux, F. \& Collin, M. Origin, homeostasis and function of Langerhans cells and other langerin-expressing dendritic cells. Nature Reviews Immunology 8, 935-947 (2008).

34. Guilliams, M. et al. Alveolar macrophages develop from fetal monocytes that differentiate into long-lived cells in the first week of life via GM-CSF. J. Exp. Med. 210, 1977-1992 (2013).

35. Amit, I., Winter, D. R. \& Jung, S. The role of the local environment and epigenetics in shaping macrophage identity and their effect on tissue homeostasis. Nat Immunol 17, 18-25 (2016).

36. Haldar, M. et al. Heme-mediated SPI-C induction promotes monocyte differentiation into iron-recycling macrophages. Cell 156, 1223-1234 (2014).

37. Dixon, L. J., Barnes, M., Tang, H., Pritchard, M. T. \& Nagy, L. E. Kupffer cells in the liver. Compr Physiol 3, 785-797 (2013).

38. Teitelbaum, S. L. \& Ross, F. P. Genetic regulation of osteoclast development and function. Nat. Rev. Genet. 4, 638-649 (2003).

39. Anjos Cassado, dos, A., Lima, M. R. D. \& Bortoluci, K. R. Revisiting Mouse Peritoneal Macrophages: Heterogeneity, Development, and Function. Frontiers in Immunology 6, 2 (2015).

40. Prinz, M. \& Priller, J. Microglia and brain macrophages in the molecular 
age: from origin to neuropsychiatric disease. Nat Rev Neurosci 15, 300312 (2014).

41. Galea, I., Bechmann, I. \& Perry, V. H. What is immune privilege (not)? Trends in Immunology 28, 12-18 (2007).

42. Abbott, N. J., Patabendige, A. A. K., Dolman, D. E. M., Yusof, S. R. \& Begley, D. J. Structure and function of the blood-brain barrier. Neurobiology of Disease 37, 13-25 (2010).

43. Louveau, A. et al. Structural and functional features of central nervous system lymphatic vessels. Nature 523, 337-341 (2015).

44. Zlokovic, B. V. Neurovascular pathways to neurodegeneration in Alzheimer's disease and other disorders. Nat Rev Neurosci (2011). doi:10.1038/nrn3114

45. Kierdorf, K. et al. Microglia emerge from erythromyeloid precursors via Pu.1- and Irf8-dependent pathways. Nat Neurosci 16, 273-280 (2013).

46. Ginhoux, F. et al. Fate mapping analysis reveals that adult microglia derive from primitive macrophages. Science 330, 841-845 (2010).

47. Tanaka, Y. et al. Early ontogenic origin of the hematopoietic stem cell lineage. Proceedings of the National Academy of Sciences of the United States of America 109, 4515-4520 (2012).

48. Tober, J., Yzaguirre, A. D., Piwarzyk, E. \& Speck, N. A. Distinct temporal requirements for Runx1 in hematopoietic progenitors and stem cells. Development 140, 3765-3776 (2013).

49. Schulz, C. et al. A lineage of myeloid cells independent of Myb and hematopoietic stem cells. Science 336, 86-90 (2012).

50. Butovsky, O. et al. Identification of a unique TGF- $\beta$-dependent molecular and functional signature in microglia. Nat Neurosci 17, 131-143 (2014).

51. Waisman, A., Ginhoux, F., Greter, M. \& Bruttger, J. Homeostasis of Microglia in the Adult Brain: Review of Novel Microglia Depletion Systems. Trends in Immunology 36, 625-636 (2015).

52. Goldmann, T. et al. Origin, fate and dynamics of macrophages at central nervous system interfaces. Nat Immunol (2016). doi:10.1038/ni.3423

53. Nimmerjahn, A., Kirchhoff, F. \& Helmchen, F. Resting microglial cells are highly dynamic surveillants of brain parenchyma in vivo. Science 308, 1314-1318 (2005).

54. Paolicelli, R. C. et al. Synaptic pruning by microglia is necessary for normal brain development. Science 333, 1456-1458 (2011).

55. Marín-Teva, J. L. et al. Microglia promote the death of developing Purkinje cells. Neuron 41, 535-547 (2004).

56. Zhan, Y. et al. Deficient neuron-microglia signaling results in impaired functional brain connectivity and social behavior. Nat Neurosci 17, 400-406 (2014).

57. Cardona, A. E. et al. Control of microglial neurotoxicity by the fractalkine receptor. Nat Neurosci 9, 917-924 (2006).

58. Ransohoff, R. M. \& Cardona, A. E. The myeloid cells of the central nervous system parenchyma. Nature 468, 253-262 (2010).

59. Parkhurst, C. N. et al. Microglia Promote Learning-Dependent Synapse Formation through Brain-Derived Neurotrophic Factor. Cell 155, 1596-1609 (2013).

60. Tremblay, M.-È., Lowery, R. L. \& Majewska, A. K. Microglial interactions with synapses are modulated by visual experience. PLoS Biol. 8, e1000527 
(2010).

61. Biber, K., Neumann, H., Inoue, K. \& Boddeke, H. W. G. M. Neuronal 'On' and "Off" signals control microglia. Trends in Neurosciences 30, 596-602 (2007).

62. Carmelina Gemma, A. D. B. The role of microglia in adult hippocampal neurogenesis. Front. Cell. Neurosci. 7, 229 (2013).

63. Ekdahl, C. T., Claasen, J.-H., Bonde, S., Kokaia, Z. \& Lindvall, O. Inflammation is detrimental for neurogenesis in adult brain. Proceedings of the National Academy of Sciences of the United States of America 100, 13632-13637 (2003).

64. Saijo, K. \& Glass, C. K. Microglial cell origin and phenotypes in health and disease. Nature Reviews Immunology 11, 775-787 (2011).

65. Prinz, M., Tay, T. L., Wolf, Y. \& Jung, S. Microglia: unique and common features with other tissue macrophages. Acta Neuropathol 128, 319-331 (2014).

66. Hemmer, B., Archelos, J. J. \& Hartung, H.-P. New concepts in the immunopathogenesis of multiple sclerosis. Nat Rev Neurosci 3, 291-301 (2002).

67. Cua, D. J. et al. Interleukin-23 rather than interleukin-12 is the critical cytokine for autoimmune inflammation of the brain. Nature 421, 744-748 (2003).

68. Becher, B., Durell, B. G. \& Noelle, R. J. IL-23 produced by CNS-resident cells controls $T$ cell encephalitogenicity during the effector phase of experimental autoimmune encephalomyelitis. J Clin Invest 112, 1186-1191 (2003).

69. Heppner, F. L. et al. Experimental autoimmune encephalomyelitis repressed by microglial paralysis. Nat. Med. 11, 146-152 (2005).

70. Yamasaki, R. et al. Differential roles of microglia and monocytes in the inflamed central nervous system. J. Exp. Med. 211, 1533-1549 (2014).

71. Butovsky, O. et al. Modulating inflammatory monocytes with a unique microRNA gene signature ameliorates murine ALS. J Clin Invest 122, 3063-3087 (2012).

72. Michaud, J.-P., Bellavance, M.-A., Préfontaine, P. \& Rivest, S. Real-time in vivo imaging reveals the ability of monocytes to clear vascular amyloid beta. Cell Rep 5, 646-653 (2013).

73. Mildner, A. et al. Distinct and non-redundant roles of microglia and myeloid subsets in mouse models of Alzheimer's disease. J. Neurosci. 31, 1115911171 (2011).

74. Grathwohl, S. A. et al. Formation and maintenance of Alzheimer's disease beta-amyloid plaques in the absence of microglia. Nat Neurosci 12, 13611363 (2009).

75. Krabbe, G. et al. Functional Impairment of Microglia Coincides with BetaAmyloid Deposition in Mice with Alzheimer-Like Pathology. PLoS ONE 8, e60921 (2013).

76. Xie, Z. et al. Peroxynitrite mediates neurotoxicity of amyloid beta-peptide142- and lipopolysaccharide-activated microglia. J. Neurosci. 22, 3484-3492 (2002).

77. Shechter, R. et al. Recruitment of beneficial M2 macrophages to injured spinal cord is orchestrated by remote brain choroid plexus. Immunity 38, 555-569 (2013). 
78. Perry, V. H., Nicoll, J. A. R. \& Holmes, C. Microglia in neurodegenerative disease. Nature Publishing Group 6, 193-201 (2010).

79. Salminen, A., Ojala, J., Kaarniranta, K. \& Kauppinen, A. Mitochondrial dysfunction and oxidative stress activate inflammasomes: impact on the aging process and age-related diseases. Cell. Mol. Life Sci. 69, 2999-3013 (2012).

80. Nakanishi, H. \& Wu, Z. Microglia-aging: Roles of microglial lysosome- and mitochondria-derived reactive oxygen species in brain aging. Behavioural Brain Research 201, 1-7 (2009).

81. Lee, T. I. \& Young, R. A. Transcriptional Regulation and Its Misregulation in Disease. Cell 152, 1237-1251 (2013).

82. Blackwood, E. M. \& Kadonaga, J. T. Going the distance: a current view of enhancer action. Science 281, 60-63 (1998).

83. Bennett, M. L. et al. New tools for studying microglia in the mouse and human CNS. Proceedings of the National Academy of Sciences of the United States of America 113, E1738-E1746 (2016).

84. Koso, H. et al. Conditional rod photoreceptor ablation reveals Sall1 as a microglial marker and regulator of microglial morphology in the retina. Glia (2016). doi:10.1002/glia.23038

85. Sweetman, D. \& Münsterberg, A. The vertebrate spalt genes in development and disease. Dev. Biol. 293, 285-293 (2006).

86. Nishinakamura, R. et al. Murine homolog of SALL1 is essential for ureteric bud invasion in kidney development. Development 128, 3105-3115 (2001).

87. Lauberth, S. M. \& Rauchman, M. A Conserved 12-Amino Acid Motif in Sall1 Recruits the Nucleosome Remodeling and Deacetylase Corepressor Complex. Journal of Biological Chemistry 281, 23922-23931 (2006).

88. Sato, A. et al. Sall1, a causative gene for Townes-Brocks syndrome, enhances the canonical Wnt signaling by localizing to heterochromatin. Biochemical and Biophysical Research Communications 319, 103-113 (2004).

89. Netzer, C., Bohlander, S. K., Rieger, L., Müller, S. \& Kohlhase, J. Interaction of the developmental regulator SALL1 with UBE2I and SUMO-1. Biochemical and Biophysical Research Communications 296, 870-876 (2002).

90. de Celis, J. F. \& Barrio, R. Regulation and function of Spalt proteins during animal development. Int. J. Dev. Biol. 53, 1385-1398 (2009).

91. Harrison, S. J., Nishinakamura, R., Jones, K. R. \& Monaghan, A. P. Sall1 regulates cortical neurogenesis and laminar fate specification in mice: implications for neural abnormalities in Townes-Brocks syndrome. Disease Models \& Mechanisms 5, 351-365 (2012).

92. Kohlhase, J. SALL1 mutations in Townes-Brocks syndrome and related disorders. Hum. Mutat. 16, 460-466 (2000).

93. Vodopiutz, J. et al. Homozygous SALL1 mutation causes a novel multiple congenital anomaly-mental retardation syndrome. J. Pediatr. 162, 612-617 (2013).

94. Ginhoux, F. \& Prinz, M. Origin of Microglia: Current Concepts and Past Controversies. Cold Spring Harbor Perspectives in Biology 7, a020537 (2015).

95. Takasato, M. et al. Identification of kidney mesenchymal genes by a combination of microarray analysis and Sall1-GFP knockin mice. 
Mechanisms of Development 121, 547-557 (2004).

96. Abedin, M. J., Imai, N., Rosenberg, M. E. \& Gupta, S. Identification and Characterization of Sall1-Expressing Cells Present in the Adult Mouse Kidney. Nephron Exp Nephrol 119, e75-e82 (2011).

97. Hirsch, S. et al. A mouse model of Townes-Brocks syndrome expressing a truncated mutant Sall1 protein is protected from acute kidney injury. Am. J. Physiol. Renal Physiol. 309, F852-63 (2015).

98. Jung, S. et al. Analysis of fractalkine receptor CX(3)CR1 function by targeted deletion and green fluorescent protein reporter gene insertion. Mol. Cell. Biol. 20, 4106-4114 (2000).

99. Nicholas H Varvel, S. A. G. F. B. C. L. A. B. B. B. D. R. T. I. F. C. F. L. H. A. A. O. G. R. M. R. M. J. Microglial repopulation model reveals a robust homeostatic process for replacing CNS myeloid cells. Proceedings of the National Academy of Sciences of the United States of America 109, 18150 (2012).

100. Bruttger, J. et al. Genetic Cell Ablation Reveals Clusters of Local SelfRenewing Microglia in the Mammalian Central Nervous System. Immunity 43, 92-106 (2015).

101. Elmore, M. R. P. et al. Colony-stimulating factor 1 receptor signaling is necessary for microglia viability, unmasking a microglia progenitor cell in the adult brain. Neuron 82, 380-397 (2014).

102. Schreiner, B., Heppner, F. L. \& Becher, B. Modeling multiple sclerosis in laboratory animals. Semin Immunopatho/ 31, 479-495 (2009).

103. Croxford, A. L. et al. The Cytokine GM-CSF Drives the Inflammatory Signature of CCR2+ Monocytes and Licenses Autoimmunity. Immunity 43, 502-514 (2015).

104. Inoue, S., Inoue, M., Fujimura, S. \& Nishinakamura, R. A mouse line expressing Sall1-driven inducible Cre recombinase in the kidney mesenchyme. genesis NA-NA (2010). doi:10.1002/dvg.20603

105. Remington, L. T., Babcock, A. A., Zehntner, S. P. \& Owens, T. Microglial recruitment, activation, and proliferation in response to primary demyelination. The American Journal of Pathology 170, 1713-1724 (2007).

106. Chiu, I. M. et al. A neurodegeneration-specific gene-expression signature of acutely isolated microglia from an amyotrophic lateral sclerosis mouse model. Cell Rep 4, 385-401 (2013).

107. Goldmann, T. et al. A new type of microglia gene targeting shows TAK1 to be pivotal in CNS autoimmune inflammation. Nat Neurosci 16, 1618-1626 (2013).

108. Surace, M. J. \& Block, M. L. Targeting microglia-mediated neurotoxicity: the potential of NOX2 inhibitors. Cell. Mol. Life Sci. 69, 2409-2427 (2012).

109. Ikushima, H., Negishi, H. \& Taniguchi, T. The IRF Family Transcription Factors at the Interface of Innate and Adaptive Immune Responses. Cold Spring Harb Symp Quant Biol 78, 105-116 (2013).

110. Ivashkiv, L. B. \& Donlin, L. T. Regulation of type I interferon responses. Nature Reviews Immunology 14, 36-49 (2014).

111. Kimura, A. et al. Aryl hydrocarbon receptor protects against bacterial infection by promoting macrophage survival and reactive oxygen species production. Int. Immunol. 26, 209-220 (2014).

112. Esser, C., Rannug, A. \& Stockinger, B. The aryl hydrocarbon receptor in immunity. Trends in Immunology 30, 447-454 (2009). 
113. Fourgeaud, L. et al. TAM receptors regulate multiple features of microglial physiology. Nature 532, 240-244 (2016).

114. Kempermann, G., Jessberger, S., Steiner, B. \& Kronenberg, G. Milestones of neuronal development in the adult hippocampus. Trends in Neurosciences 27, 447-452 (2004).

115. Ginhoux, F. \& Jung, S. Monocytes and macrophages: developmental pathways and tissue homeostasis. Nature Reviews Immunology 14, 392404 (2014).

116. Hoeffel, G. et al. Adult Langerhans cells derive predominantly from embryonic fetal liver monocytes with a minor contribution of yolk sacderived macrophages. J. Exp. Med. 209, 1167-1181 (2012).

117. de Celis, J. F., Barrio, R. \& Kafatos, F. C. A gene complex acting downstream of dpp in Drosophila wing morphogenesis. Nature 381, 421424 (1996).

118. Kanda, S. et al. Sall1 maintains nephron progenitors and nascent nephrons by acting as both an activator and a repressor. J. Am. Soc. Nephrol. 25, 2584-2595 (2014).

119. Yamashita, K., Sato, A., Asashima, M., Wang, P.-C. \& Nishinakamura, R. Mouse homolog of SALL1, a causative gene for Townes?Brocks syndrome, binds to $A / T$-rich sequences in pericentric heterochromatin via its $C$ terminal zinc finger domains. Genes Cells 12, 171-182 (2007).

120. Bruel-Jungerman, E., Rampon, C. \& Laroche, S. Adult hippocampal neurogenesis, synaptic plasticity and memory: facts and hypotheses. Rev Neurosci 18, 93-114 (2007).

121. Zhang, Y. et al. Purification and Characterization of Progenitor and Mature Human Astrocytes Reveals Transcriptional and Functional Differences with Mouse. Neuron 89, 37-53 (2016).

122. Yuri, S. et al. Sall4 is essential for stabilization, but not for pluripotency, of embryonic stem cells by repressing aberrant trophectoderm gene expression. Stem Cells 27, 796-805 (2009).

123. Li, J., Chen, K., Zhu, L. \& Pollard, J. W. Conditional deletion of the colony stimulating factor-1 receptor (c-fms proto-oncogene) in mice. genesis 44, 328-335 (2006).

124. Levéen, P. et al. TGF-beta type II receptor-deficient thymocytes develop normally but demonstrate increased CD8+ proliferation in vivo. Blood 106, 4234-4240 (2005).

125. Buch, T. et al. A Cre-inducible diphtheria toxin receptor mediates cell lineage ablation after toxin administration. Nat Meth 2, 419-426 (2005).

126. Codarri, L. et al. RORyt drives production of the cytokine GM-CSF in helper $T$ cells, which is essential for the effector phase of autoimmune neuroinflammation. Nat Immunol 12, 560-567 (2011).

127. Greter, M. et al. GM-CSF controls nonlymphoid tissue dendritic cell homeostasis but is dispensable for the differentiation of inflammatory dendritic cells. Immunity 36, 1031-1046 (2012). 